DISSERTATION

\title{
A THEORY OF TOPOGRAPHICALLY BOUND BALANCED MOTIONS AND APPLICATION TO ATMOSPHERIC LOW-LEVEL JETS
}

\author{
Submitted by \\ Levi Glenn Silvers \\ Department of Atmospheric Science \\ In partial fulfillment of the requirements \\ for the Degree of Doctor of Philosophy \\ Colorado State University \\ Fort Collins, Colorado
}

Fall 2011

Doctoral Committee:

Advisor: Wayne H. Schubert

David A. Randall

David W.J. Thompson

Richard E. Eykholt 


\section{ABSTRACT}

\section{A THEORY OF TOPOGRAPHICALLY BOUND BALANCED MOTIONS AND APPLICATION TO ATMOSPHERIC LOW-LEVEL JETS}

The response of a stratified fluid to forcing from the lower boundary is studied both analytically and numerically. The lower boundary forces a flow field through orographic obstacles and potential vorticity anomalies. It is argued that these mechanisms contribute to the maintenance of low-level jets (LLJs) that are observed regularly in the vicinity of the Rocky Mountains and the Andes. Low-level jets function as one of the primary mechanisms through which topography and surface heating influence regional and global climates.

On the $f$-plane a horizontal transform of the governing equation for potential vorticity leads to a vertical structure equation that is solved using Green's functions. On the sphere a vertical transform of this system leads to a horizontal structure equation that is solved using spheroidal harmonics. These analytic solutions lead to a conceptually simple picture of the fluid response to forcing. However, these derivations only lead to closed analytic solutions for the case of an isentropic lower boundary. When the lower boundary is not isentropic a massless layer must be included in the domain and the solution is then found iteratively. For the cases including a massless layer the system is approximated using finite differences and solved with an over-relaxation procedure.

Solutions are presented for the geostrophically balanced, steady response of the fluid to three idealized lower boundaries. An isentropic ridge is studied to determine the role non-heated orography plays on the wind field. Then a flat heated lower boundary and a non-isentropic ridge are 
studied. The cases with a heated lower surface result in a cyclonic wind field that is anchored over the topography. Observations show a prominent cyclonic wind field centered on both the Rocky Mountains and the Andes. The idealized cases studied in this work allow for the examination of fluid systems analogous to the Great Plains LLJ and the South American LLJ. Both the mean behavior of these jets and their variability have important climatological and economic impacts on the plains regions of North and South America.

One of the purposes of this work is to interpret particular LLJ systems as part of the orographically bound, balanced motion associated with the potential vorticity anomalies produced by solar heating. This research proposes the jets on opposite sides of the mountains to be a single response to potential vorticity forcing that is the result of radiative heating on the Rockies and the Andes. Although the importance of heated orography to LLJs has tended to be downplayed in the literature, it is shown here to be a significant component in the maintenance of LLJs. 


\section{ACKNOWLEDGEMENTS}

I would first like to express thanks to all who have contributed to my education up to this point. I did not arrive here on my own. The department of atmospheric science at Colorado State University has provided a great environment in which I have learned much. My advisor Wayne Schubert has contributed an enormous amount to my education in general, and specifically the research presented here. His patience and insight have served as good teachers.

My family also has played a central role throughout my education. My wife Brittany has provided limitless support, my parents have encouraged me and given me the opportunity to succeed, and my siblings have offered further support and friendship.

The number of people at Colorado State University who have helped me is too long to list exhaustively. However, a few of those especially deserving mention are Rick Taft, Brian McNoldy, Paul Ciesielski, Jonathan Vigh, Matt Masarik, and Gabriel Williams as well as the rest of the Schubert research group. My committee has given helpful direction to my research projects. In addition to my committee Professors Takamitsu Ito and Thomas Birner and Scott Fulton have offered valuable insight.

The funding for this research project was generously provided by the Center for Multiscale Modeling of Atmospheric Processes.

Last but not least, the Thursday Morning Coffee Group has provided much needed friendship and regular coffee consumption for at least the past four years. 


\section{TABLE OF CONTENTS}

1 Introduction 1

1.1 General Motivation . . . . . . . . . . . . . . . . . . . . . 1

1.2 Influence of low-level jets on regional and global climates . . . . . . . . . . 10

1.3 Research hypothesis . . . . . . . . . . . . . . . . . . . . . 12

2 Observations of Low-Level Jets 15

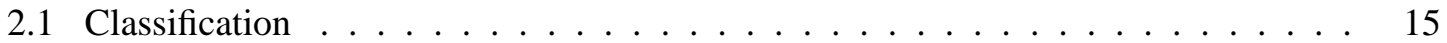

2.2 Data Coverage . . . . . . . . . . . . . . . . . . . 18

2.3 North and South American Low-Level Jets . . . . . . . . . . . . . . . . . . 21

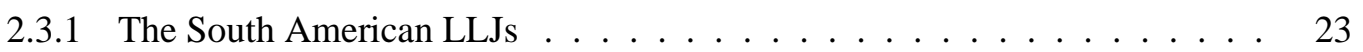

2.3.2 The North American LLJs . . . . . . . . . . . . . . . . . . . 25

2.3 .3 Summary . . . . . . . . . . . . . . . . . . . 28

3 Dynamical theory of topographically bound balanced motions on the $f$-plane 29

3.1 Introduction . . . . . . . . . . . . . . . . . . . . . 29

3.2 Basic governing equations $\ldots \ldots \ldots \ldots \ldots \ldots \ldots \ldots \ldots \ldots$

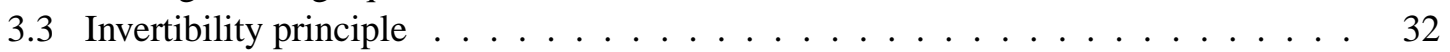

3.4 Fourier transform of the invertibility principle . . . . . . . . . . . . . . . 34

3.5 Solution of the vertical structure equation . . . . . . . . . . . . . . 37

3.6 Critical crest height . . . . . . . . . . . . . . . . . . . . 42

4 Topographically Bound Balanced Motions on the Sphere 45

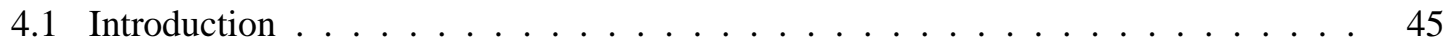

4.2 Invertibility principle . . . . . . . . . . . . . . 45

4.3 Solution of the invertibility principle on the $f$-plane via transform methods . . . . 47

4.4 The case of an isentropic mountain . . . . . . . . . . . . . . . . . . . . 49

4.5 Invertibility principle on the sphere . . . . . . . . . . . . . 50

4.6 Solution of the invertibility problem via transform methods on the sphere . . . . 52

4.7 Discussion of various solution methods . . . . . . . . . . . . . 54

5 Numerical Solutions $\quad 57$

5.1 Comparison between analytic and numerical solutions . . . . . . . . . . . . 57

5.1.1 Finite difference discretization and successive over-relaxation . . . . . . . 58

5.2 Results . . . . . . . . . . . . . . . . . . . . 62

5.3 Technical Discussion . . . . . . . . . . . . . . . . . 70 
6 Conclusions $\quad 71$

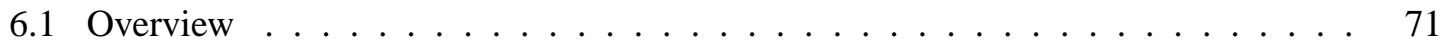

6.2 Summary of wind field response to forcing . . . . . . . . . . . . . 72

6.3 Final Discussion . . . . . . . . . . . . . . . . . . . . . . 74

Bibliography 76 


\section{FIGURES}

1.1 Schematic representing three idealized fluid configurations to be studied. Contours represent lines of constant potential temperature (isentropes), the vertical axis could be either height or pressure. On the left is an isentropic obstacle, the middle is a heated flat lower surface, and the right is a heated obstacle. . . . . . . . . . . .

1.2 January 2003 mean $925 \mathrm{hPa}$ wind (scale of $10 \mathrm{~m} \mathrm{~s}^{-1}$ vector indicated just below the colorbar) from the NCEP-DOE Reanalysis II data (Kanamitsu et al. 2002), which has the horizontal/vertical resolution T62/L28. Vectors are not drawn in the region of the Andes above $925 \mathrm{hPa}$ (at T62 horizontal resolution). The background map shows topography (see colorbar, scale is 100's m), with the La Plata river basin outlined in red. Adapted from Tarasova et al. (2006) and Noguès-Paegle et al. (2001).

1.3 The meridional wind component (shading; $\mathrm{m} \mathrm{s}^{-1}$ ) at 03 local time for (a) the North American Regional Reanalysis (NARR), (b) the GFDL atmospheric general circulation model (AGCM) with orography, and (c) the identical AGCM with continentality but with the orography reduced to zero. Vectors show the summer mean wind, scale of $8 \mathrm{~m} \mathrm{~s}^{-1}$ is in upper right corner. From Jiang et al. (2007). . . . . . . . . .

2.1 Isotachs $\left(\mathrm{m} \mathrm{s}^{-1}\right)$ along the southwest to northeast cross-section running from (21.5S, $63.5 \mathrm{~W})$ to $(19.2 \mathrm{~S}, 58.7 \mathrm{~W})$. The data was obtained between 15:00 and 16:20 UTC on 6 February 2003 (during SALLJEX) by the sawtooth flight trajectory of one of the NOAA P-3 aircraft. From Vera et al. (2006). . . . . . . . . . . . . . . . . . .

2.2 21-yr mean NLLJ index and 500m AGL wind field for January and July calculated from hourly analyses. The scale of a $10 \mathrm{~m} \mathrm{~s}^{-1}$ wind vector is indicated in bottom right of January figure. See text for details. From Rife et al. 2010. . . . . . . . . .

2.3 Wind profile vs height from a high-resolution research rawinsonde (solid), a regular NWS rawinsonde (dashed), and the NWS averaged high-resolution rawinsonde (dotted). From Stensrud et al. 1990. . . . . . . . . . . . . . . . . . . .

2.4 Mean warm season reanalysis meridional wind field at $21 \mathrm{~S}$ from the Year of Tropical Convection. The magnitude of wind is given by colorbar, black contours are

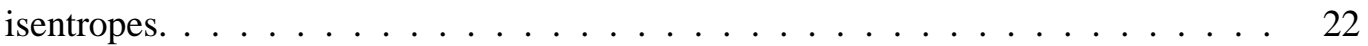

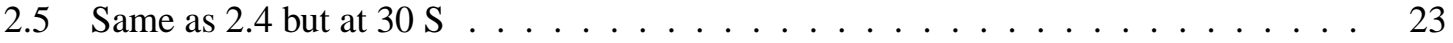

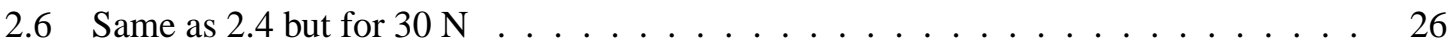

2.7 Same as 2.4 but for $35 \mathrm{~N} \ldots \ldots \ldots \ldots \ldots \ldots \ldots$

3.1 Pressure as a function of $\theta$ as computed from (3.27) for reference state 1 (solid curve) and for reference state 2 (dashed curve) . . . . . . . . . . . . . 38

3.2 The Green's function for select values of $\kappa \theta_{B}$. For this figure $\theta^{\prime}=305 K \ldots \ldots$ 
3.3 Wind field for an isentropic lower boundary. The Gaussian halfwidth of the mountain is $500 \mathrm{~km}$, the maximum height is $\mathrm{H}=1700 \mathrm{~m}$, and the latitude is $30 \mathrm{~N}$. Solid black contours are the isentropes. . . . . . . . . . . . . . . . . 41

3.4 Critical crest height as a function of latitude for reference state $1 \ldots \ldots$. . . . . 43

3.5 Critical crest height as a function of Gaussian halfwidth for reference state 1 at five different latitudes. . . . . . . . . . . . . . . . . . . . . . . . . 44

4.1 Vertical structure functions $V_{\ell}(\theta)$ for $\ell=0,1,2,3 \ldots \ldots \ldots \ldots$

5.1 Meridional wind field (shading, warm colors are positive, cool colors negative, contour interval $=1 \mathrm{~m} / \mathrm{s}$ ) for an isentropic lower boundary computed using overrelaxation. The maximum height of the Gaussian mountain is $1700 \mathrm{~m}$ and its halfwidth $=500 \mathrm{~km}$. The maximum winds are $14.3 \mathrm{~m} / \mathrm{s} . \ldots \ldots \ldots$

5.2 Meridional wind field (shading, $1 \mathrm{~m} / \mathrm{s}$ intervals) for an isentropic ridge with $H$ $=1800 \mathrm{~m}, v_{\max }=15.4 \mathrm{~m} / \mathrm{s}$. Black contours are isentropes (top) and isobars (bottom). 63

5.3 Meridional wind field (shading, $1 \mathrm{~m} / \mathrm{s}$ intervals) for a heated flat lower surface with a $6 \mathrm{~K}$ temperature anomaly and Gaussian halfwidth $\mathrm{a}=600 \mathrm{~km}, v_{\max }=12.7 \mathrm{~m} / \mathrm{s}$. The massless layer in $(x, p)$-space (top) is indicated by black object along lower boundary, in $(x, \theta)$-space (bottom) it is indicated by the thick black line. Contour spacing in the massless layer is $1 \mathrm{hPa} \ldots \ldots \ldots \ldots$

5.4 Meridional wind field (shading, $1 \mathrm{~m} / \mathrm{s}$ intervals) for a heated ridge with a $6 \mathrm{~K}$ temperature anomaly, $H=1800 \mathrm{~m}, a=600 \mathrm{~km}, v_{\max }=11.13 \mathrm{~m} / \mathrm{s}$. The massless layer is indicated by the black object (top) and the thick black line (bottom). Halfwidth of ridge is $900 \mathrm{~km}$. Contour spacing in the massless layer is $50 \mathrm{hPa}$ except for lowest

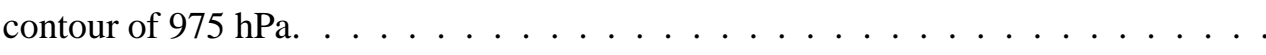

5.5 Same as Fig. 5.3 except with a $12 \mathrm{~K}$ temperature anomaly, $v_{\max }=27.27 \mathrm{~m} / \mathrm{s}$. Contour spacing in the massless layer is $4 \mathrm{hPa} \ldots \ldots \ldots \ldots 6$

5.6 Same as Fig. 5.4 except with a $12 \mathrm{~K}$ temperature anomaly, $v_{\max }=10.3 \mathrm{~m} / \mathrm{s}$. . . 


\section{TABLES}

4.1 The spectrum of gravity wave speeds $c_{\ell}$, and approximate gravity wave speeds for the values of $\ell$ listed in the left column. The exact values of $c_{\ell}$ have been computed from (4.13) and the approximate values from (4.14) using $\theta_{B}=295 \mathrm{~K}, \theta_{T}=360$ $\mathrm{K}, g=9.81 \mathrm{~m} \mathrm{~s}^{-2}$, and $\left(\theta_{B} / g\right) N_{B}^{2}=5.1373 \mathrm{~K} \mathrm{~km}^{-1} \ldots \ldots \ldots$ 


\section{Chapter 1}

\section{INTRODUCTION}

\subsection{General Motivation}

How does a stratified fluid respond to an obstacle in its path? The fluid will either pass over or move around the sides of the obstacle. The details of the resulting fluid motion will depend on the initial velocity and stratification of the fluid as well as the size, shape, and temperature of the obstacle. Although this may seem to be an overly simplified scenario, it has important implications for the fluid motions that are observed in the atmosphere and oceans as a response to the complex orography of the Earth. The atmosphere is strongly stratified and is certainly influenced by numerous large mountain ranges as the Earth rotates. Notable examples of prominent mountains that are known to have major impacts on the climate and weather are the Rocky Mountains, the Andes, the mass of land and ice in Greenland, and the Tibetan plateau. All of these orographic features experience seasonal and diurnal fluctuations in surface temperature which also serve to influence the atmospheric flow. The research presented here explores the general response of a stratified fluid to both isentropic and non-isentropic orography.

The steady, topographically bound balanced response of a fluid to orography was studied by Eliassen (1980). His classification for isentropic flows used the following method. Begin by assuming the lowest isentropic surface (a surface of constant entropy) follows the lower boundary in the far field. Isentropic obstacles are then defined as those whose geopotential surface continuously follows an isentropic surface. A non-isentropic obstacle (Eliassen's 'warm' obstacle) is one whose geopotential surface protrudes through one or more isentropes, usually at a summit. These two 

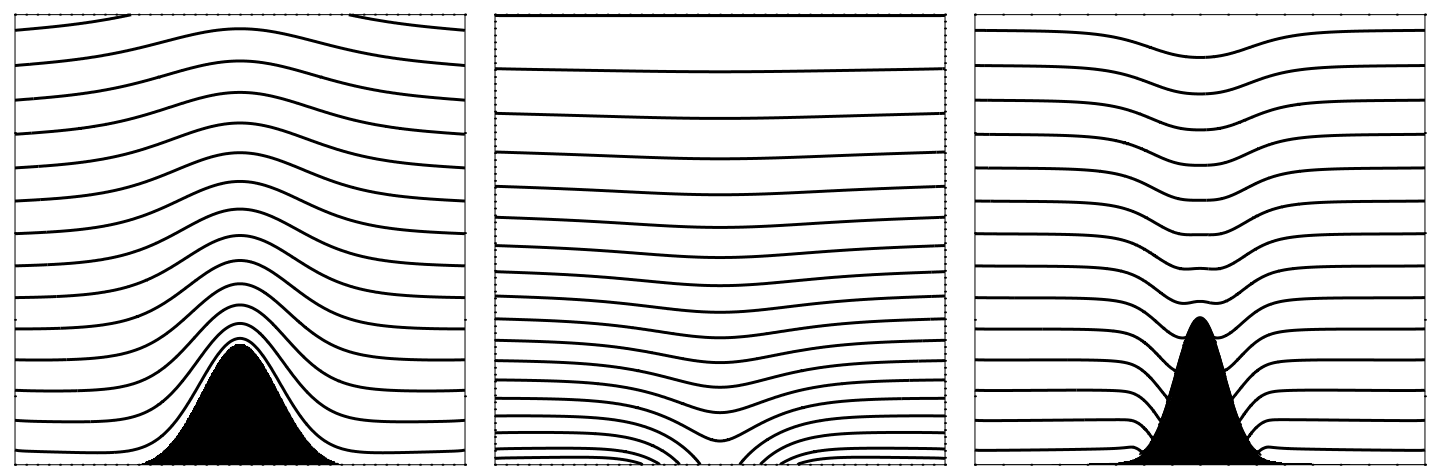

Figure 1.1: Schematic representing three idealized fluid configurations to be studied. Contours represent lines of constant potential temperature (isentropes), the vertical axis could be either height or pressure. On the left is an isentropic obstacle, the middle is a heated flat lower surface, and the right is a heated obstacle.

types of obstacles represent fundamentally different distributions of entropy within the fluid domain. Eliassen showed that solutions for an isentropic ridge were only possible if the height of that ridge did not exceed a critical value. If this 'critical crest height' was exceeded, the ridge must be a warm obstacle. Because Eliassen's paper did not address a heated lower boundary his warm obstacles always corresponded to objects that exceed the critical crest height. However, orographic features that are radiatively heated also satisfy his criterion for a warm obstacle even if their crest height does not exceed the critical crest height. Non-heated warm obstacles are characterized by isentropes that are compressed over the summit, with the lowest isentropic surfaces bending up as they intersect the lower boundary. In contrast, heated obstacles are characterized by isentropes that are stretched out over the summit with the isentropic surfaces bending down as they intersect the lower boundary as in the right panel of Fig. 1.1.

The impact of three simple lower boundary conditions on the atmospheric wind and mass fields is studied in this dissertation. Figure 1.1 shows a schematic representation of these three cases. On the left is an isentropic ridge which was studied by Eliassen (1980). The center panel represents a heated lower surface with a constant geopotential. A combination of these two cases results in a heated obstacle as shown in the right panel. The contours are isentropes, or lines of 
constant potential temperature. Isentropes that intersect with the lower boundary indicate a temperature gradient along that boundary and can represent a 'heated' boundary. When considering a fluid in hydrostatic and geostrophic balance that conserves potential vorticity, compressed isentropic surfaces result in anticyclonic motion, while stretched isentropic surfaces lead to cyclonic motion. Thus in the case of an isentropic obstacle there will be anticyclonic vorticity over the summit where the isentropes are compressed and cyclonic vorticity in the surroundings. When the lower boundary is heated the interpretation of the isentropes is more complicated because potential vorticity is not conserved. However, the results of this research and observations shown in the next chapter indicate that, even with a heated lower boundary, compressed isentropes indicate anticyclonic vorticity while vertically stretched isentropes lead to cyclonic vorticity.

Figures 1.2 and 1.3 show the mean circulation around both the Andes and the Rocky Mountains to be dominated by cyclonic flow. Embedded within this flow around the Andes are the South American Low-Level Jet (SALLJ) to the east, and the Chilean Low-Level Coastal Jet (CLLCJ) to the west. Similarly, the cyclonic flow around the Rocky Mountains includes the Great Plains LowLevel Jet (GPLLJ) and the California Coastal Low-Level Jet (CCLLJ). This observed mean flow suggests that there could be a connection between the flow generated by a heated ridge and the persistent low-level jets on either side of these mountain ranges.

Relatively fast moving confined currents of air in the lower atmosphere are consistently observed in the vicinity of many mountainous regions. Numerous atmospheric phenomena with some form of a horizontal wind field maximum in the lower troposphere have been referred to as lowlevel jets (LLJs). Bonner (1968) developed a number of characteristics that have often been used to define the North American Great Plains Low-Level Jet (GPLLJ) and more recently the South American Low-Level Jet (SALLJ). Many of the common definitions for LLJs include the following three characteristics in the wind field: a horizontal wind maximum in the lower $2 \mathrm{~km}$ of the troposphere, a moderate to high vertical and/or horizontal gradient in the wind field, and a measurable diurnal oscillation. One of the results of this work is to interpret particular low-level jet systems as part of the orographically bound, balanced motion associated with the potential vorticity anomalies 
produced by solar heating.

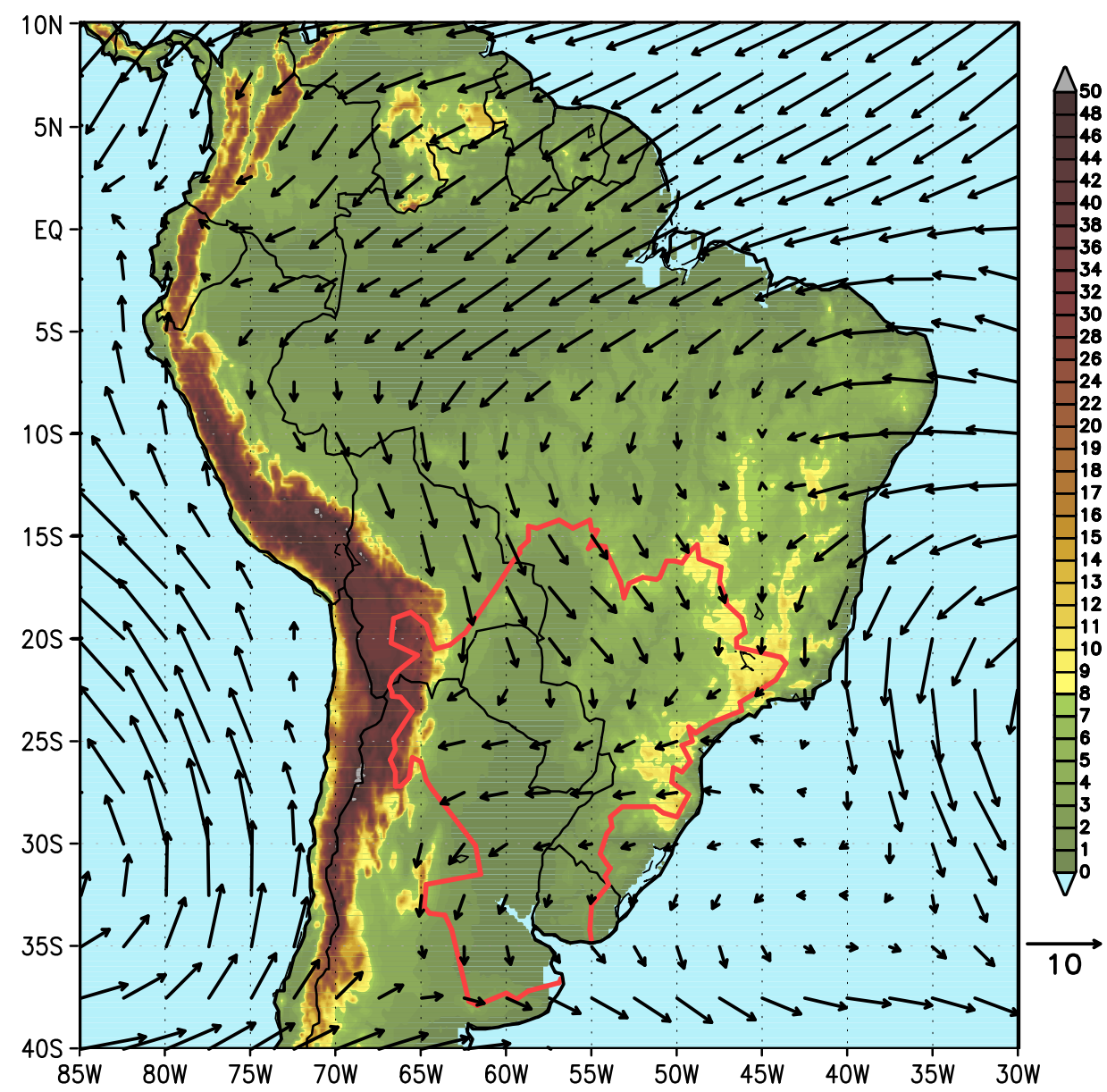

Figure 1.2: January 2003 mean $925 \mathrm{hPa}$ wind (scale of $10 \mathrm{~m} \mathrm{~s}^{-1}$ vector indicated just below the colorbar) from the NCEP-DOE Reanalysis II data (Kanamitsu et al. 2002), which has the horizontal/vertical resolution T62/L28. Vectors are not drawn in the region of the Andes above 925 $\mathrm{hPa}$ (at T62 horizontal resolution). The background map shows topography (see colorbar, scale is 100's m), with the La Plata river basin outlined in red. Adapted from Tarasova et al. (2006) and Noguès-Paegle et al. (2001).

Descriptions of low-level jets began to appear in the literature as early as the 1930s (Farquharson 1939). At the time very little was understood about these atmospheric motions and almost no relevant data was available. Research picked up in the fifties as the strong influence of LLJs on precipitation was realized. In spite of sparse data the early studies did a reasonable job of identifying the dominant characteristics of the LLJs. (see Means 1952, Hoecker 1963, Bonner 1968, Hoxit 1975, and references therein). Most of the initial focus was on the GPLLJ but the past couple of 
decades have seen a dramatic growth of interest in other LLJs around the globe (Vera et al., 2006; Jiang et al., 2010; Rife et al., 2010; and references therein).

As the basic characteristics of LLJs became more clear, additional questions arose as to why the jets existed. What physical explanation could explain such regularly occurring but highly variable phenomena? Some of the first studies to propose specific physical mechanisms responsible for the generation and maintenance of the jets were those by Buajitti and Blackadar (1957), Blackadar (1957), Wexler (1961) and Holton (1967). Blackadar's approach focuses on the daily oscillation of solar heating. During the day radiation from the sun heats the Earth's surface and leads to the generation of wind as a response to pressure gradients. Because of large friction near the surface, the boundary layer wind quickly becomes turbulent, which implies a high value of the eddy viscosity. After sunset, radiatively driven boundary layer turbulence ceases and the eddy viscosity decreases dramatically. The wind in the LLJ is often observed to become supergeostrophic as it is shielded from boundary layer friction. Blackadar hypothesized that this leads to an oscillation in the total wind field as a result of the oscillation of the ageostrophic wind in response to the Coriolis force (an inertial oscillation). This would lead to a northward wind maximum in the early morning.

In 1961 Wexler attributed the location of the GPLLJ to a simple deflection by the mountains of Central America of the winds in the Bermuda high pressure system and considered the GPLLJ as analogous to the Atlantic gulf stream. He argued that the local effects of radiation and friction used in Blackadar's argument were not enough to create the highly concentrated wind field in the LLJ but that it was fundamentally due to large scale inertial forces. Similarly, deflection of the trade winds over South America by the Andes has been proposed as one of several possible mechanisms responsible for the SALLJ (Vera et al., 2006). According to Holton (1967) scale analysis can show that the GPLLJ is not a close analogue to oceanic western boundary currents. However, a mechanical deflection of wind currents by the mountains does play an important role in the life cycle of the American LLJs. The coastal LLJs in particular are strongly impacted by the Pacific high pressure systems.

Holton (1967) examined the oscillations of a density field over uniformly heated gently slop- 
ing terrain (e.g. the Great Plains). The resulting mechanism he proposed as responsible for the GPLLJ can be illustrated by looking at the variation of the pressure gradient force between the day and night. During the day relatively high regions of the terrain will be heated more than air at the same altitude that is not at the surface. This uneven heating leads to a pressure gradient force directed towards the higher elevation. East of the Rocky Mountains this results in an westward flow which is turned northward by the Coriolis force. Jiang et al. (2007) provide a helpful analysis of Holton's theory and show the output from the Geophysical Fluid Dynamics Laboratory (GFDL) atmospheric general circulation model (AGCM). They show (see their Fig. 5) the zonal pressure gradient force, air temperature, and wind fields averaged over the latitudes $30 \mathrm{~N}$ to $40 \mathrm{~N}$ at 1800 Local Time (LT). The pressure gradient force is clear on both sides of the Rocky Mountains and forces upslope wind. An inertial oscillation due to the Coriolis force acting to the right of the motion, if given enough time, will result in a northward flow east of the mountains. During the night, radiative cooling of the orography reverses this process with an eventual southward flow. These flows resulting from the heating add to the mean summertime northward wind over the Great Plains. Thus the mean flow is enhanced by the northward flow at night and decreased by the southward flow during the day.

Jiang et al. (2007) also compared the AGCM to the North American Regional Reanalysis to investigate the relative importance of the mechanisms proposed by Blackadar and Holton. They found that both mechanisms are necessary for producing a GPLLJ that compares well with observations. Blackadar's theory accounts well for the diurnal oscillation but not the location of the GPLLJ. Wexler's theory accounts well for the location but not the strength or diurnal oscillation and Holton's theory does reasonably well with both the location and strength of the LLJ but struggles with the vertical structure and phase. Holton's approach also does not account for the uneven surface heating which is clearly occuring each day. Another weakness of Blackadar's theory is that if the GPLLJ is largely due to the inertial oscillation that is forced by changes in eddy viscosity then we should observe similar jets everywhere that eddy viscosity changes periodically. We do not observe this; instead LLJs are almost always found near some prominent topographic feature such as a mountain 
range or a coastline. This suggests that orography may serve to concentrate or amplify the affects considered by Blackadar.

South American mean January 2003 winds at $925 \mathrm{mb}$ are shown in Figure 1.2. A striking feature is the strong cyclonic flow centered around the Andes. The South American Low-Level Jet is seen in the southward flow east of the Andes and the Chilean Low-Level coastal jet is clear in the northward flow over the eastern Pacific Ocean. The La Plata river basin is outlined in red. The SALLJ has a major impact on the South American hydrologic cycle, particularly in the La Plata basin. This basin extends over parts of five countries and supports a significant production of food and hydroelectric energy. Understanding the spatial extent, strength, and variability of the SALLJ is therefore critical for the regional economies of several different countries.

Figure 1.3 (from Jiang et al. 2007) shows the climatological summertime mean wind vectors at $925 \mathrm{mb}$. The top panel (1.3a) shows the North American Regional Reanalysis (NARR). The NARR is a combination of the National Centers for Environmental Prediction mesoscale Eta forecast model and data from October 1978 to December 2003. The resolution of NARR is $32 \mathrm{~km}$ with 45 vertical layers and output every 3 hours. For more information see Jiang et al. (2007). The middle panel (1.3b) shows a control run by the GFDL atmospheric general circulation model (realistic topography, full physics model with resolution of $1^{\circ}$ latitude by $1.25^{\circ}$ longitude and 24 vertical levels), and the lower panel (1.3c) shows another run by the GFDL model but with the orography removed.

In general there is very good agreement between the NARR (1.3a) and the model (1.3b). The model captures well the location and spatial structure of the GPLLJ and the jet off the coast of California. The model does not however calculate the correct magnitude of the subtropical high in the western Atlantic or the maximum in the LLJ wind field over the Gulf coast. The model also does not capture the correct wind field in the Gulf of California. When the orography is removed, the low-level structure in the wind field is dramatically altered. There are no longer any LLJs; the wind fields in the eastern Pacific and over the southern Great Plains are fairly uniform. The wind maxima that are present have been greatly weakened and spread out. This figure clearly shows the 
important affect of orography on the LLJs.

These two figures strongly suggest that the Rocky Mountains and the Andes impact the formation and maintenance of the GPLLJ, the SALLJ and the LLJs off the west coasts of California and Chile. The orographic impact can best be understood as having several components. Possibly the most obvious impact is the unchanging shape of the mountains that protrude into the atmosphere forcing the wind to change course. As shown in this dissertation low-level jets are also influenced through the radiative heating that occurs at the surface of the mountains. Another indirect effect on the LLJs comes from latent heating as a result of weather induced by the mountains. When looking at observations and model output it is not easy to determine which of these effects, if any, dominate or how they interact with each other. 

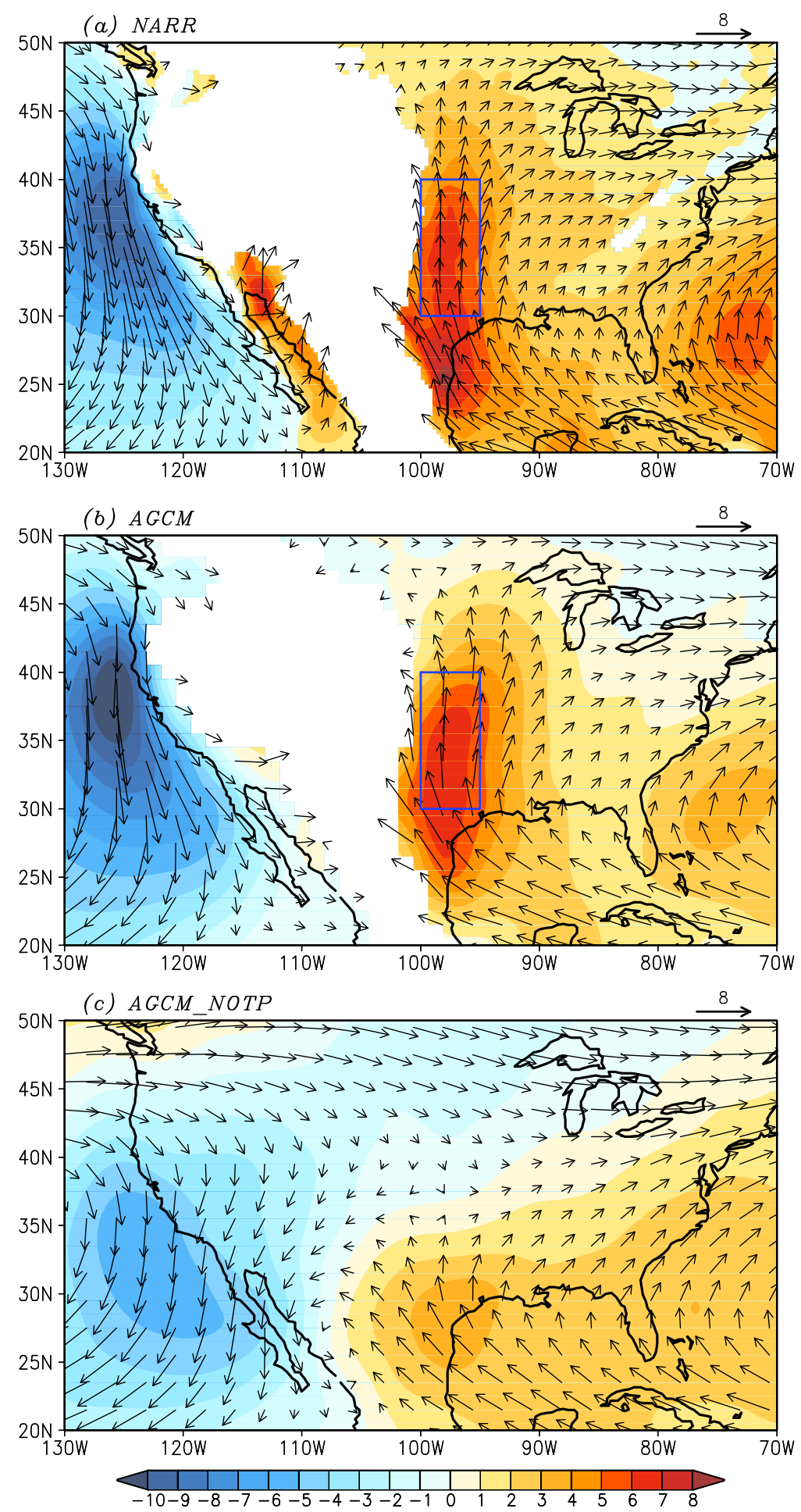

Figure 1.3: The meridional wind component (shading; $\mathrm{m} \mathrm{s}^{-1}$ ) at 03 local time for (a) the North American Regional Reanalysis (NARR), (b) the GFDL atmospheric general circulation model (AGCM) with orography, and (c) the identical AGCM with continentality but with the orography reduced to zero. Vectors show the summer mean wind, scale of $8 \mathrm{~m} \mathrm{~s}^{-1}$ is in upper right corner. From Jiang et al. (2007). 


\subsection{Influence of low-level jets on regional and global climates}

Persistent and strong low-level jets have a major impact on the regional climates in both North and South America. Both the GPLLJ and SALLJ transport large amounts of moisture from low tropical latitudes to the interiors of the continents and benefit agricultural production. The largest annual flux of moisture into the interior of North America occurs during the summer months and is attributed to the GPLLJ (Higgins et al. 1997). A significant amount of the moisture necessary for the agriculturally productive La Plata basin in South America is transported there by the SALLJ (Marengo et al. 2004, Vera et al. 2006). There are also persistent coastal jets off the west coasts of the Americas. The coastal jets strongly influence the weather patterns and radiation budgets of the regions and lead to intense oceanic coastal upwelling. This upwelling is a vital component of the coastal economies and leads to rich marine ecosystems (Jiang et al. 2010). The California coastal LLJ (CCLLJ) has been the subject of many studies while the Chilean Low-Level Coastal Jet (CLLCJ) has received less attention. Due to the excellent data now available for the Great Plains LLJ and the significant economic impact of both the GPLLJ and the South American LLJ, these two LLJs have been studied much more than other documented LLJs around the globe.

Not only do the American LLJs directly impact the moisture budget, they have also been closely tied to the formation of large regions in the atmosphere that are favorable to deep convection and mesoscale convective systems (MCSs). This is accomplished by the jets importing large amounts of warm, humid air from lower latitudes, enhancing upward motion in the lower troposphere and creating instabilities that often lead to convective activity. This most often occurs downstream of the LLJ maxima in the so-called exit region of the jet. Thus the seasonal fluctuations in the strength and location of LLJs can influence the distribution of deep convective events (Stensrud 1996, Vera et al. 2006). This connection between LLJs and deep convective activity is one of their clearest connections to regional and global climates.

Mesoscale large convective systems (MCSs) impact the global climate in several ways. They produce a high proportion of the global precipitation. The vertical gradient of heating produced by MCSs strongly influences the atmospheric large-scale flow field (Houze, 2004). They influence 
the radiation budget through the production of abundant high-level clouds and the surface energy budget through latent heat fluxes. The global distribution of lightning has been shown to correlate highly with the distribution of MCSs (see Houze 2004, fig. 47). Through their strong influence on MCSs, LLJs thus indirectly impact many components of the global climate.

Mesoscale convective complexes (MCCs) are a subset of MCSs. They occur primarily over land, tend to be quasi-circular, and are usually quite intense (Maddox, 1980). It has been hypothesized that their high intensity is in part due to large low-level buoyancy over land that results from the daytime radiative heating. However, over land the boundary layer becomes stable at night and does not generally provide a favorable environment for MCCs to form or intensify. This should lead to a clear minimum of MCSs over land because they need more than the 10 to 12 hours of favorable boundary layer conditions that are provided by daytime heating. This is distinctly not what the observations show. In fact, in the subtropics and midlatitudes almost all of the MCCs observed by Laing and Fritsch (1997, see Houze Fig. 46) occurred over land, not the ocean. Figure 46 of Houze (2004) also clearly shows the regions where MCCs preferentially form to be the same regions of North and South America that experience LLJs. These LLJs, which reach their maximum strength at night, bring copious amounts of warm, moist air from the tropics into the Great Plains and the La Plata basin making the nighttime boundary layer in these regions more favorable for the maintenance of MCSs than they would be otherwise. This is the mechanism through which the spatial distribution of MCSs over Earth is heavily influenced by LLJs. It is therefore reasonable to conclude that LLJs should not be ignored when modeling the Earth's climate (Stensrud 1996).

Of the many studies on LLJs, two papers by Byerle and Paegle $(2002,2003)$ are among only a few that mention the cyclonic circulation over the Rockies and the Andes. The focus of these studies is on the interaction between the orography and the mean zonal flow. They demonstrate how orography can function as a scale transfer mechanism to focus global scale patterns into regional responses. This can potentially lead to increased predictability for extreme LLJ events. The barotropic vorticity equation is used to study the possible impact of stationary Rossby waves on the weather patterns over the plains regions of North and South America. 
Spatial and temporal variability of low-level jets are perhaps the most important characteristics for those living in regions directly impacted by LLJs. For example, if the mean center of a LLJ shifts by $70 \mathrm{~km}$ the regional rainfall pattern would also shift and could result in the rainfall for a particular region significantly changing. Similarly, if the month in which the LLJ occured most frequently changed the resulting regional weather would most likely change as well. Both of these effects strongly impact agriculture because of the influence on precipitation rates during the growing season for a particular area.

Low-level jets can be thought of as regionally and globally forced meso-synoptic features of the general circulation that impact local climates. There are LLJs throughout the atmosphere with a wide spectrum of characteristics that are influenced by many different factors. The dominant patterns of variability and structure of a particular jet are usually due to the physical mechanisms that are forcing and maintaining that jet. We know that natural seasonal fluctuations in these jets influences regional temperatures and precipitation but it is usually not clear exactly how. Trying to understand the natural variability of LLJs and any possible future changes in their behavior is a major current challenge.

\subsection{Research hypothesis}

The hypothesis for the research presented in this dissertation is that the balanced atmospheric response to a heated lower boundary significantly influences the formation and maintenance of lowlevel jets. Specifically, this work examines the dynamically balanced response of a stratified fluid to obstacles and temperature anomalies on the lower boundary. This is relevant to multiple regions of the globe that experience persistent low-level jets. It is proposed that the jets on opposite sides of the North and South American mountains are part of a single response to potential vorticity forcing that is the result of radiative heating at the surface of the Rocky Mountains and the Andes. The results imply that the Great Plains LLJ and the California coastal LLJ are two components of the same response to diabatic heating of the Rocky Mountains and similarly that the South American LLJ and the LLJ off the coast of Chile are part of the same response to heating of the Andes. Each 
of these four jets has been previously studied, but almost exclusively in isolation. The dynamical approach taken here provides a unified view for understanding the topographically bound balanced motions that are important to the life cycle of LLJs.

Although the work of Eliassen (1980) was not specifically applied to LLJs it is relevant to them and provides physical insight into the dynamical characteristics of these prevalent phenomena. His paper laid the theoretical groundwork for this research, but did not address applications to LLJs or find solutions for non-isentropic objects as is done here.

Three simplified cases are mathematically studied. Figure 1.1 gives a schematic picture of these cases. An isentropic ridge for which no isentropes intersect the lower boundary, a flat (constant geopotential surface) lower boundary with a non-constant lower isentropic surface, and a non-isentropic ridge with isentropes intersecting its surface. These three cases describe well many observed scenarios in the atmosphere including a temperature gradient along a flat lower boundary and heated orography.

Isentropic surfaces are used as the vertical coordinate because of their convenient conservation properties for adiabatic processes. A material element of fluid will not cross an isentropic surface unless it is diabatically heated. This allows for simple interpretations of the potential vorticity dynamics. Isentropic surfaces that intersect the ground are one of the primary difficulties this coordinate system leads to because it is challenging to correctly formulate the lower boundary condition. Incorporating a massless layer and slightly modifying the necessary transforms to and from spectral space allows for a consistent physical domain with isentropic surfaces and a geopotential lower surface that is intersected by isentropes.

Although LLJs have been studied extensively for decades, to the best of the author's knowledge, the approach presented here is unique and leads to new insights concerning the forcing and variability of these jets. Orographic affects have been known to impact the LLJs for as long as they have been studied. The precise impact of simple orographic heating has rarely been quantified; this research develops an analytic theory for this and applies it to the atmosphere. This allows for the determination of how the heated orography influences the wind and mass field apart from other 
forcing mechanisms.

In short, this dissertation presents the solutions governing the response of a stratified fluid to a heated lower boundary. The primary question motivating this research is whether heating of the Rocky Mountains and the Andes plays a significant role in the generation and maintenance of low-level jets. One of the results is the ability to directly compute the impact of changing diabatic forcing on the lower boundary.

The next chapter describes relevant observations of low-level jets. Chapter 3 lays out the dynamical system to be studied and derives solutions on the $f$-plane. A critical crest height is derived above which it is no longer mathematically possible for an object to be isentropic. The wind field is plotted for an isentropic ridge using these analytic solutions. Chapter 4 is similar to chapter 3 but the solution of the invertibility principle is generalized to a sphere. The solutions derived in chapters 3 and 4 are only closed solutions for the case of no heating along the lower boundary. In order to compute the wind and mass fields that result from heated lower boundaries chapter 5 solves the invertibility principle using finite-difference approximations of the governing equations. An analysis is then made of the results and how they compare to general observations of LLJs. Final conclusions and a discussion of the implications of this research are given in chapter 6 . 


\section{Chapter 2}

\section{OBSERVATIONS OF LOW-LEVEL JETS}

\subsection{Classification}

A precise classification of a low-level jet is difficult because of the plethora of phenomena that have been referred to as LLJs. The term has been used for regions of high speed horizontal wind having intense diurnal fluctuations, and for regions with no diurnal fluctuations. High speed wind fields that result from mountain gaps and extend for roughly $200 \mathrm{~km}$ were referred to as LLJs (Macklin et al., 1990) as well as the synoptic scale Great Plains LLJ ( on the order of 1000 $\mathrm{km}$ ). Arakawa (1956) has discussed wind patterns at $500 \mathrm{mb}$ as LLJs while the height of the wind maximum described by Macklin et al. (1990) was at just $80 \mathrm{~m}$ above ground level (AGL). If a maximum in the wind field at any height, with unspecified shear characteristics, and a horizontal extent that ranges from the small meso-scale to the synoptic scale can be referred to as a LLJ then the atmospheric phenomena that fall under the term LLJ will be so diverse and numerous that a general explanation of the dominant forcing mechanisms and variability should not be expected.

Of the many LLJ definitions that have been used, one that has become prevalent is that proposed by Bonner (1968) in his study of the GPLLJ. He required the height of the wind maximum AGL to be no greater than $1.5 \mathrm{~km}$ (this is the same height used by Blackadar (1957)). In addition a hierarchy of criteria were defined based on the magnitude of the wind. As taken directly from Bonner (1968) these criteria are as follows.

(1) The wind speed at the level of maximum wind must equal or exceed $12 \mathrm{~m} / \mathrm{s}$ and must decrease by at least $5 \mathrm{~m} / \mathrm{s}$ to the next higher minimum or to the $3 \mathrm{~km}$ level, whichever is 
lower.

(2) The wind speed at the level of maximum wind must equal or exceed $16 \mathrm{~m} / \mathrm{s}$ and must decrease by at least $8 \mathrm{~m} / \mathrm{s}$ to the next higher minimum or to the $3 \mathrm{~km}$ level, whichever is lower.

(3) The wind speed at the level of maximum wind must equal or exceed $20 \mathrm{~m} / \mathrm{s}$ and must decrease by at least $10 \mathrm{~m} / \mathrm{s}$ to the next higher maximum or to the $3 \mathrm{~km}$ level, whichever is lower.

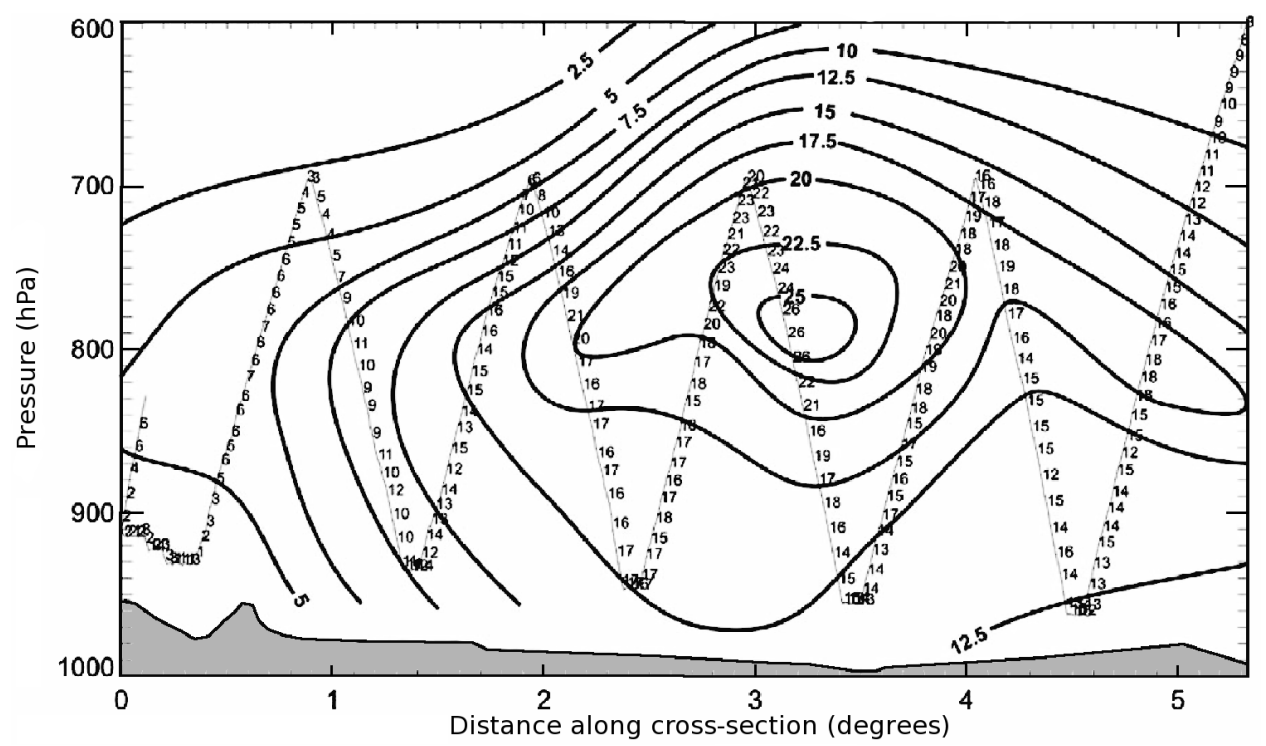

Figure 2.1: Isotachs $\left(\mathrm{m} \mathrm{s}^{-1}\right)$ along the southwest to northeast cross-section running from $(21.5 \mathrm{~S}$, $63.5 \mathrm{~W})$ to $(19.2 \mathrm{~S}, 58.7 \mathrm{~W})$. The data was obtained between 15:00 and 16:20 UTC on 6 February 2003 (during SALLJEX) by the sawtooth flight trajectory of one of the NOAA P-3 aircraft. From Vera et al. (2006).

These conditions indicate that the stronger the wind speed maximum is for a LLJ the greater the vertical shear will be. Bonner did not enforce a requirement that the GPLLJ exhibit a diurnal cycle, but the diurnal nature of the jet was clear in his observations (he also did not specify anything about the horizontal shear of the jet). The work of Reiter (1963) suggests that jets with a strong diurnal component that are connected to the nocturnal inversion should be referred to as 'inversion wind maxima' rather than LLJs, although this term has not been fully adopted in the literature. Reiter goes on to say that the term low-level jet should refer to wind fields that have a strong 
horizontal and vertical gradient and be noticeably impacted by the Coriolis force (this eliminates strong low-level regional flows). The reality is that often the term low-level jet as used in the literature is purely based on the vertical profile of the horizontal wind field (such as those shown in Figure 2.3).

The South American Low-Level Jet Experiment ( SALLJEX) chose to adopt the criteria of Bonner (1968) for their studies of the SALLJ. Thus the magnitude and the vertical profile of the horizontal wind was the primary focus while the horizontal gradient was not. Figure 2.1 shows data from SALLJEX near a LLJ maxima. The zig-zag numbered line across the figure tracks the course of the aircraft which took the data. Along the bottom of the figure the orography can be seen in grey, the slight rise on the left side of the figure is the foothills of the Andes, but the primary mountain mass is not displayed. The jet in this figure has high vertical and horizontal shear in the wind field, reaching a maximum speed of $25 \mathrm{~m} / \mathrm{s}$. This provides a good example of a LLJ that fits the somewhat generic characteristics of LLJs proposed in Chapter 1.

Figure 2.2 (from Rife et al., 2010) provides a global view of the LLJs that have a strong diurnal fluctuation. Note the GPLLJ is clearly present in the July map and the SALLJ is clear in the January map. Also apparent are nocturnal LLJs on the west coast of South Africa, throughout the Asian monsoon region,just off the Somalian coast, and western Australia. This map was specifically created to view nocturnally maximizing LLJs at or near their peak times, the time is simultaneously midnight at every point on the map. The index that is plotted was calculated based on two criteria. First, the wind at $500 \mathrm{~m}$ AGL must be stronger at local midnight than local noon and second the wind speed at $500 \mathrm{~m}$ AGL must be stronger than that at $4 \mathrm{~km}$ AGL. Based on these requirements every grid point is assigned a numeric value which is then plotted as the colors in Figure 2.2, the wind field is also shown with vectors to give a basic idea of the spatial structure of the jets. All of the global LLJs that were subjectively identified by Stensrud (1996) where successfully identified using this objective technique developed by Rife et al. (2010). 


\subsection{Data Coverage}

Based on the literature, the interest in low-level jets began with the Great Plains LLJ in the 1930s. At the time there was only sparse data over that region, making it difficult to ascertain the basic characteristics of the jet. Over the past several decades, as technology has improved and interest in increasingly accurate weather forecasts has grown, the data coverage of the GPLLJ has become more regular and dense. Although data coverage has been steadily increasing in many areas, in 2010 the only "well instrumented region" in which a nocturnal LLJ occurred was the Great Plains of North America according to Rife et al. (2010). Sparse data coverage in many of the regions that regularly experience strong LLJs has made validation of LLJ model simulations difficult. Accurately observing the jets is necessary when checking how well mesoscale and global models simulate LLJs. Observations are also a necessary component for calibrating remotely sensed data sets. The connection between LLJs and MCSs as well as the transport of large amounts of water vapor by LLJs make it clear that they need to be correctly represented in GCMs, remotely sensed data, and reanalysis products.

Obtaining high quality observations of LLJs has been difficult in the past for a variety of reasons. The jets in the Americas occur in regions with moderate to low populations which often corresponds to a low number of weather stations. Surface observations alone are not sufficient, and when the vertical profile of wind is measured, it needs to be at a fairly high resolution to properly resolve a LLJ. Another difficulty is that the LLJ wind maxima rarely occur near the traditional synoptic observation times for launching rawinsondes ( 0 and 12 UTC) but rather in the early morning hours. This results in the LLJs either being significantly underestimated, or all together missed in some of the observations.

Radiosondes take direct in-situ measurements of temperature, humidity, and pressure as a function of height. When the wind data is also processed they are called rawinsondes. Radars and satellites also can provide wind measurements by tracking clouds. Radiosondes offer a more direct and precise measurement, but they are limited in space and time because they represent the atmospheric positions at a single horizontal location and time. Radars and satellites offer much 


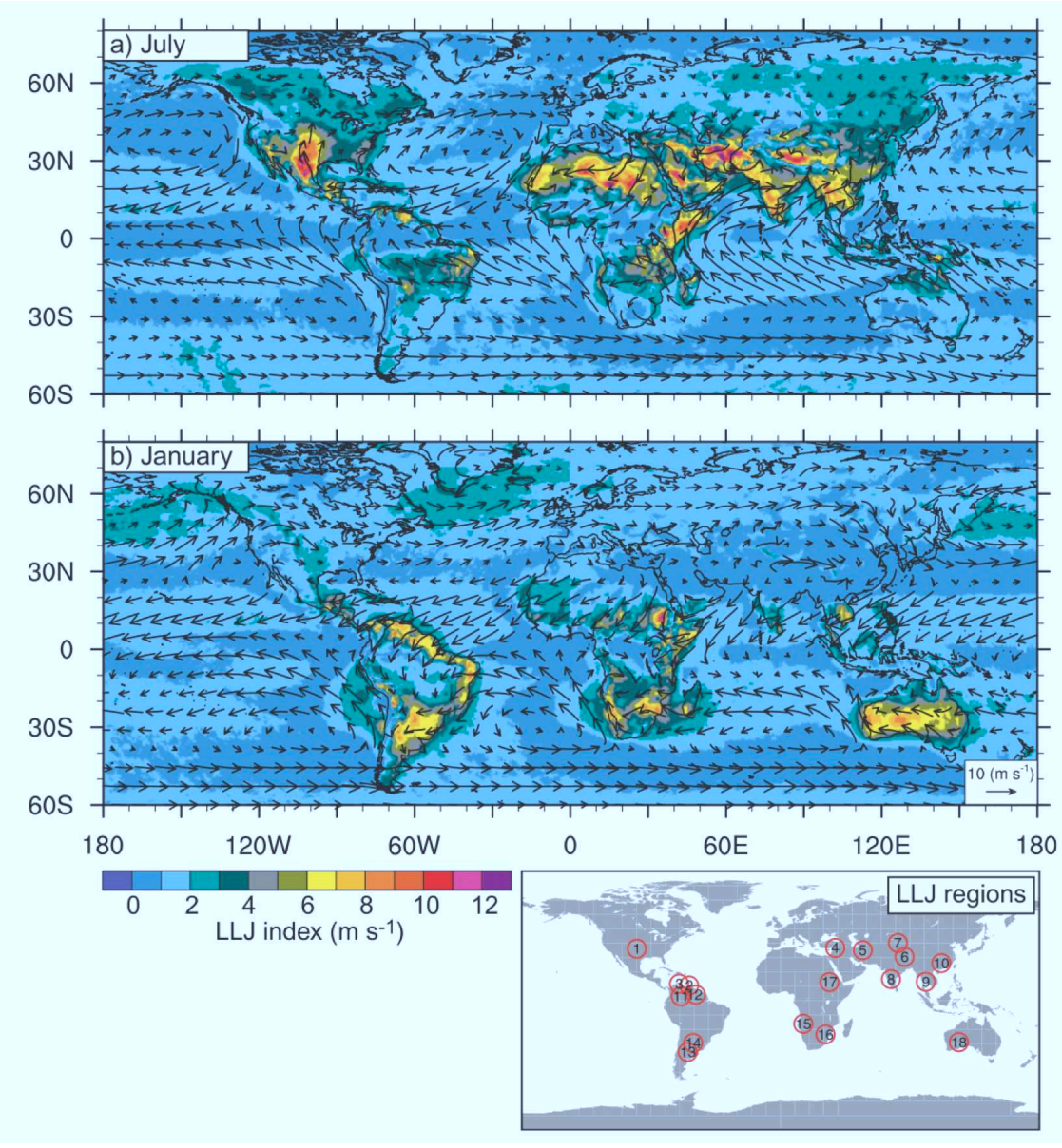

Figure 2.2: 21-yr mean NLLJ index and 500m AGL wind field for January and July calculated from hourly analyses. The scale of a $10 \mathrm{~m} \mathrm{~s}^{-1}$ wind vector is indicated in bottom right of January figure. See text for details. From Rife et al. 2010.

better spatial and temporal coverage, but they usually need verification by an external data source and can only observe atmospheric conditions when measurable aerosols are present.

Many of the radar systems currently in use have difficulty resolving the lower structure of LLJs. The NOAA 404-MHz radars only begin taking wind measurements at 500m AGL, but Stensrud (1996) (see his Fig. 3) shows that $46 \%$ of LLJs occurred below this height and Whiteman et al. (1997) show that over $50 \%$ of LLJs occur below 500m, making consistent and accurate LLJ observations with these radars impossible. The National Weather Service (NWS) applies an operational wind algorithm that smooths the 6-second wind data over a 2 minute interval. This significantly downgrades the temporal resolution of their radiosonde data and results in quite a different LLJ 


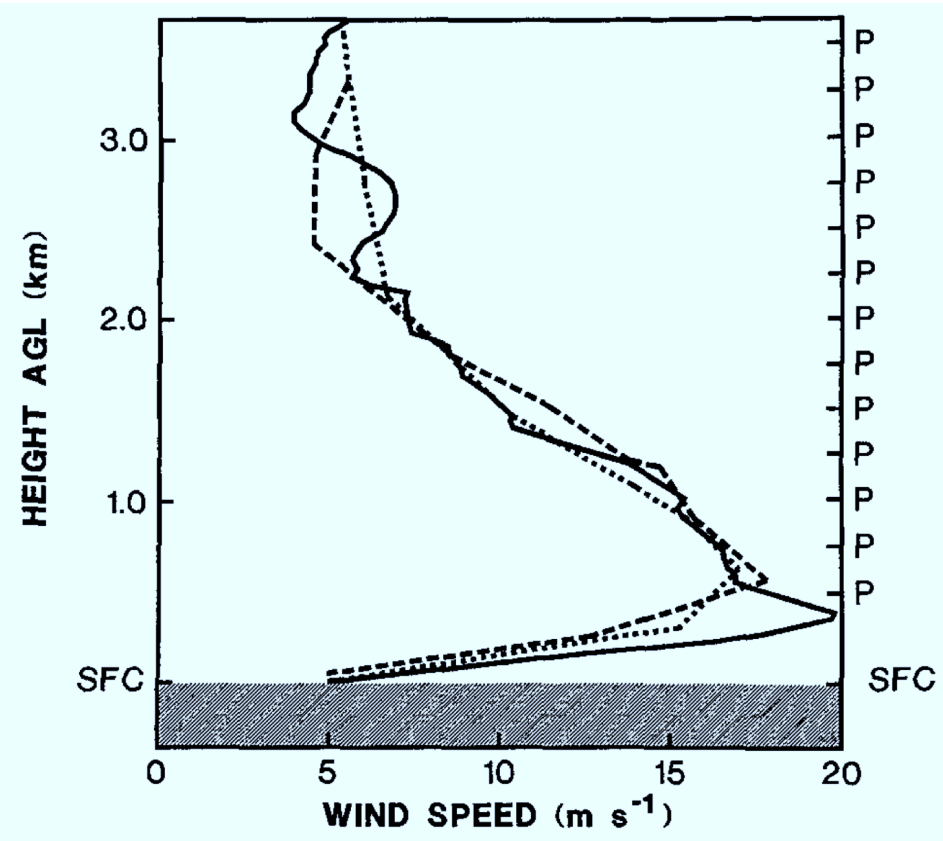

Figure 2.3: Wind profile vs height from a high-resolution research rawinsonde (solid), a regular NWS rawinsonde (dashed), and the NWS averaged high-resolution rawinsonde (dotted). From Stensrud et al. 1990.

profile than is given by unsmoothed raw data. Figure 2.3 was taken from Stensrud et al. (1990) and clearly illustrates this discrepancy between vertical wind profiles taken by different radiosondes or smoothed in time. The solid line shows a wind profile taken with an unaveraged high-resolution research rawinsonde. The wind sharply increases to a maximum of almost $20 \mathrm{~m} / \mathrm{s}$ below $500 \mathrm{~m}$ AGL before sharply dropping back to lower values. Note that the magnitude of the LLJ is significantly underestimated by the regular NWS rawinsonde and the averaged high-resolution rawinsonde. The height of the maximum wind is also overestimated. Another kind of radar being used in the U.S. is the WSR-88D. This produces wind profiles every half-hour, but they are only calculated over vertical intervals of $304 \mathrm{~m}$, so the vertical resolution is quite poor. It was also shown that a high degree of quality control is needed to confirm the validity of remotely sensed data, which is obviously a problem over regions with little to no in situ observations.

As part of their study of the Great Plains LLJ climatology Whiteman et al. (1997) compared data from a 404-MHz radar with that from a $915-\mathrm{MHz}$ radar. The $915-\mathrm{MHz}$ radar was found to have 
sufficient vertical resolution to resolve many LLJ characteristics but was frequently contaminated with noise produced by nocturnally migrating birds. Another source of valuable data is the National Oceanic and Atmospheric Administration Profiler Network (NPN) which consists of 35 doppler radar sites in 17 states of the central United States. These sites provide hourly vertical wind profile data and are an excellent source of data for the study of the GPLLJ. In contrast to the GPLLJ, the regions of South America with LLJs have been poorly observed. This has made it difficult to form a clear picture the SALLJ mean characteristics, particularly its natural variability.

In addition to increasing direct observations, the development of better reanalysis products has given an improved understanding of the global atmosphere and ocean systems. The global reanalysis developed by Rife et al. (2010) provides mesoscale horizontal resolution with grid spacing of $40 \mathrm{~km}$. The reanalysis hourly data allows for relatively detailed study of the spatial structure and diurnal cycles of LLJs around the globe. One of the goals of Rife et al. (2010) is to determine the underlying common features of NLLJs around the globe.

Thus throughout most of the past several decades some observations of LLJs have been available but several key issues created accuracy problems with the data. The situation is improving but it will still be quite a while before a high quality climatology of LLJs is available. Rather than focusing on climatological aspects of LLJs the next section presents observations from individual days or averages over short time periods of the LLJs in the Americas. This will help guide the choice of parameters used in the analysis of chapter 5 and instruct our interpretation of the results.

\subsection{North and South American Low-Level Jets}

The purpose of this section is to provide a simple picture of the American LLJs that is derived mostly from observations but with a few of the characteristics drawn from model forecasts. Figures 2.4-2.7 show mean reanalysis meridional wind fields and isentropes that were measured during the Year of Tropical Convection (YOTC) mission. The resolution of the reanalysis wind fields is $0.5^{\circ} \times 0.5^{\circ}$ and there are 11 irregularly spaced vertical levels. The mission occurred from May, 2008 through April, 2010. For more information see Waliser et al. (2010). Plots from the summer season 


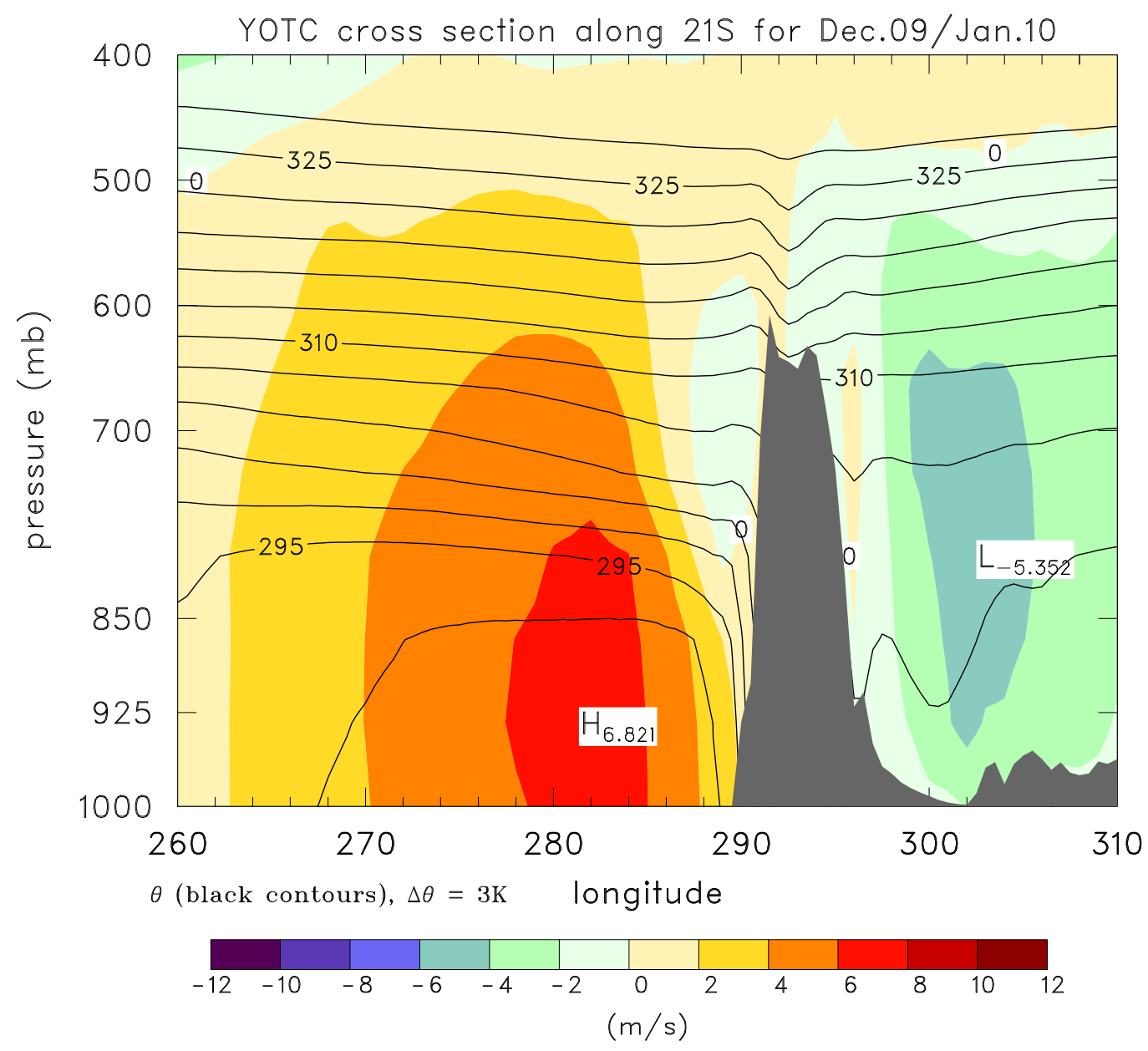

Figure 2.4: Mean warm season reanalysis meridional wind field at $21 \mathrm{~S}$ from the Year of Tropical Convection. The magnitude of wind is given by colorbar, black contours are isentropes.

in each hemisphere were chosen. Wind fields around the Andes are shown at 21S and 30S. The SALLJ often maximizes near the latitude of $20 \mathrm{~S}$, but as seen in Fig 2.5 the coastal LLJ is stronger farther south. Cross sections of the wind fields around the Rocky Mountains are shown at 30N and $35 \mathrm{~N}$. As shown in Figure 1.2 the GPLLJ maximizes at about 25N, but as seen in Figures 2.6-2.7 it is still quite strong at $30 \mathrm{~N}$ and $35 \mathrm{~N}$.

The zonally symmetric cyclonic motion centered on the mountain ranges is the most obvious feature of the wind fields in these four figures. The coastal LLJs tend to have a broader horizontal scale and a wind maxima that is closer to the surface than the jets to the east of the mountain ranges. The isentropes are generally drawn down over the mountain ranges, and intersect the surface along 


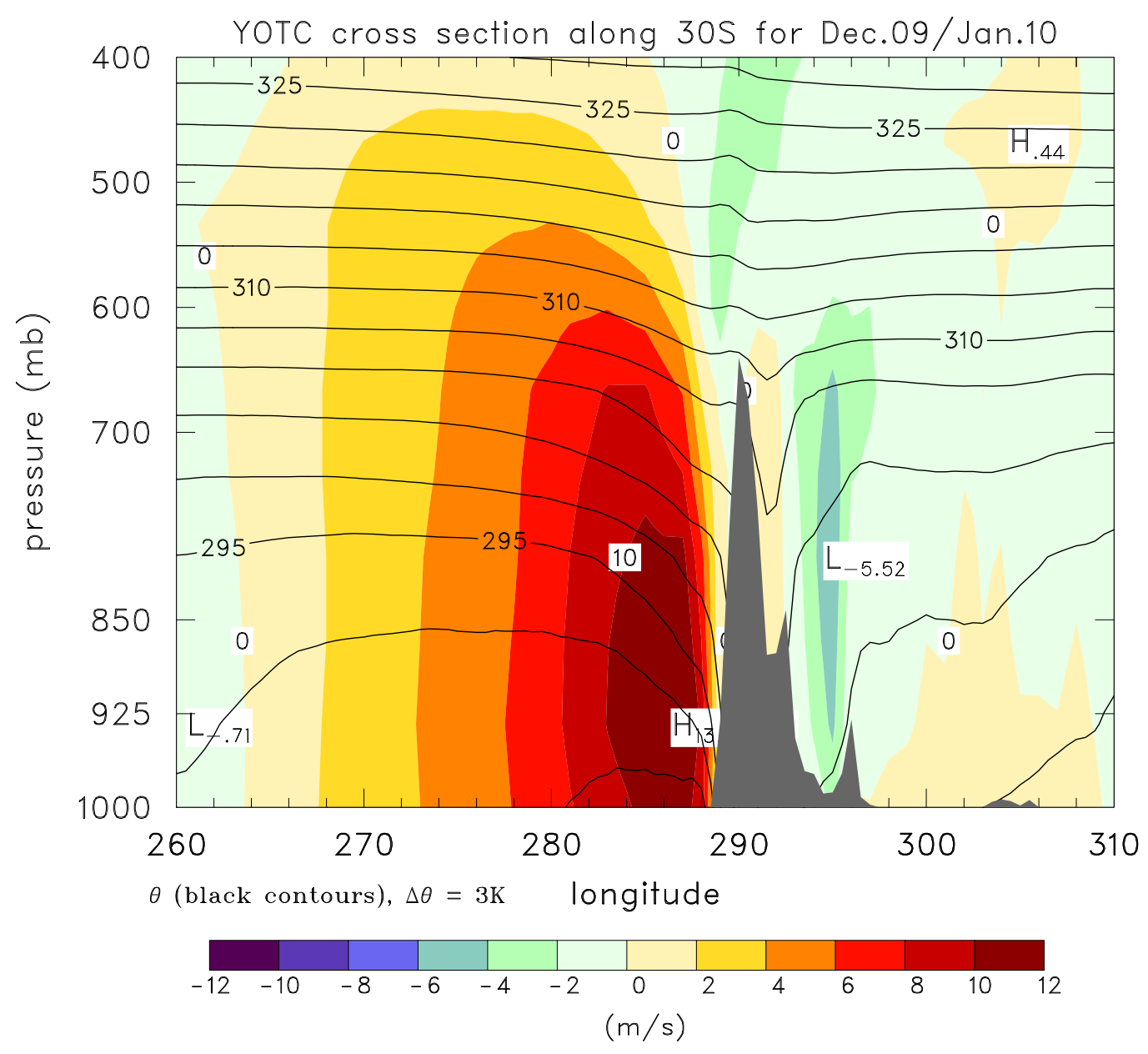

Figure 2.5: Same as 2.4 but at $30 \mathrm{~S}$

the sides and tops of the mountains. This agrees with the basic conceptual model of the potential temperature field above a heated ridge that was proposed in chapter 1 . It is also worth noting that the meridional wind is nearly zero directly over the mountain crests. For the solutions of the invertibility principle derived in chapters 3 and 4 the wind will be assumed to be zero over the ridge crest. This assumption is not necessary for the solution method of chapter 5 .

\subsubsection{The South American LLJS}

The combination of very sparse data in the region of the SALLJ, and poor resolution in the cases where data is regularly being taken has led to a poor representation of the SALLJ and the precipitation cycle in reanalysis products. The region to the east of the Andes was so poorly 
observed in the last century that the SALLJ was not identified until the 1980s based on individual case studies and a small number of rawinsonde observations. Based on Fig. 4 of Vera et al. (2006) there are no operational radiosonde sites directly to the east of the Andes between $15 \mathrm{~S}$ and $30 \mathrm{~S}$. Because this is the critical region of the SALLJ the temporary observational network that was set up for SALLJEX included six radiosonde sites and more than ten pilot balloon sites in this particular region, with additional sites in the surrounding areas. SALLJEX provided an accessible source of high quality data for the region east of the Andes during the observing period of 15 November 2002 through 15 February 2003. Radiosondes, pilot balloons, and a dense rain gauge network were used to gather data in parts of Argentina, Brazil, Paraguay, Bolivia, and Peru. The SALLJEX mission did an excellent job of determining important spatial and short term temporal characteristics of the SALLJ and supplied data that will continue to be used in future research.

Figure 2.1 shows a specific SALLJ event in February 2003 with a maximum wind around $25 \mathrm{~m} / \mathrm{s}$ at a height of $800-700 \mathrm{hPa}$. This was considered a moderate event and can be taken as representative of the SALLJ. Throughout SALLJEX the height of the maximum wind fluctuated between $500 \mathrm{~m}-3 \mathrm{~km}$. Often the height of the maximum wind will rise during the day, reflecting the deepening mixed layer with the jet 'riding' along the top. North of 20 S Saulo et al. (2000) suggest that during the warm season the SALLJ maximizes between 0600 and 1200 UTC. When the core of the jet is around $30 \mathrm{~S}$ the time of the jet maximum shifts to about six hours earlier.

During the observational period of SALLJEX there were 112 recorded MCS cases with an average lifetime of 11 hours. There were two peak times at which the MCSs reached their mature stage. This tended to occur either in the afternoon or in the night/early morning. It was shown that the maximum extent of the MCSs was reached in phase with the time of the LLJ maximum for around $70 \%$ of the cases occurring over Argentina, southern Brazil, and Uruguay. SALLJEX confirmed the diurnal variability of the LLJ wind field, but did not obtain the diurnal fluctuations of the rainfall (Vera et al. (2006)). Several previous studies had suggested that the northwestern Argentinian low (NAL) influences the southward intensification of the SALLJ, which was confirmed with SALLJEX data. 
The Chilean low-level coastal jet was studied by Jiang et al. (2010). Their paper focuses on understanding the characteristics and dynamics of the Chilean coastal LLJ. It is primarily a modeling study that used output from twice-daily 48 hour forecasts for the southeast Pacific region that were made in support of the Variability of the American Monsoon System (VAMOS) field campaign (October and November 2008). They propose that synoptic scale forcing is the primary forcing mechanism driving the Chilean coastal LLJ. The southeast Pacific high pressure system (SEPH) is the dominant pattern of the synoptic environment and its location was shown to be well correlated with the strength of the CCLLJ. The SEPH tends to move east-to-west between two preferred locations. These correspond to it being either close to or far from the coastline. When the SEPH moves closer to shore, the LLJ intensifies and the location of the wind maximum moves south.

There are striking similarities between the Chilean coastal LLJ and the California coastal LLJ. They are both directed equatorward and have similar spatial scales. The wind speed maxima for both are located between 100 and $200 \mathrm{~km}$ offshore and follow mountainous regions of coastline while being strongly influenced by the high pressure systems of the Pacific to their west. These similarities suggest that the coastal jets could be forced by similar dynamical mechanisms. Several studies (Jiang et al. 2010, Muñoz and Garreaud (2005), Chao (1985)) have hypothesized that there are three important factors that play into the forming of these two subtropical coastal jets: subtropical high pressure systems, the topography along the coast, and the land-sea differential heating.

\subsubsection{The North American LLJS}

The Great Plains low-level jet has been extensively documented and a proper review of the literature is outside the scope of this dissertation. The basic characteristics as discussed by Bonner (1968) and Whiteman et al. (1997) are briefly summarized below. Bonner studied data from 47 rawinsonde stations in the United States for the years 1959 and 1960, Whiteman et al. (1997) used data from a single station near the border of Oklahoma and Kansas during a two year period (7 April 1994 through 30 March 1996). In general the Whiteman et al. (1997) study had much better vertical 


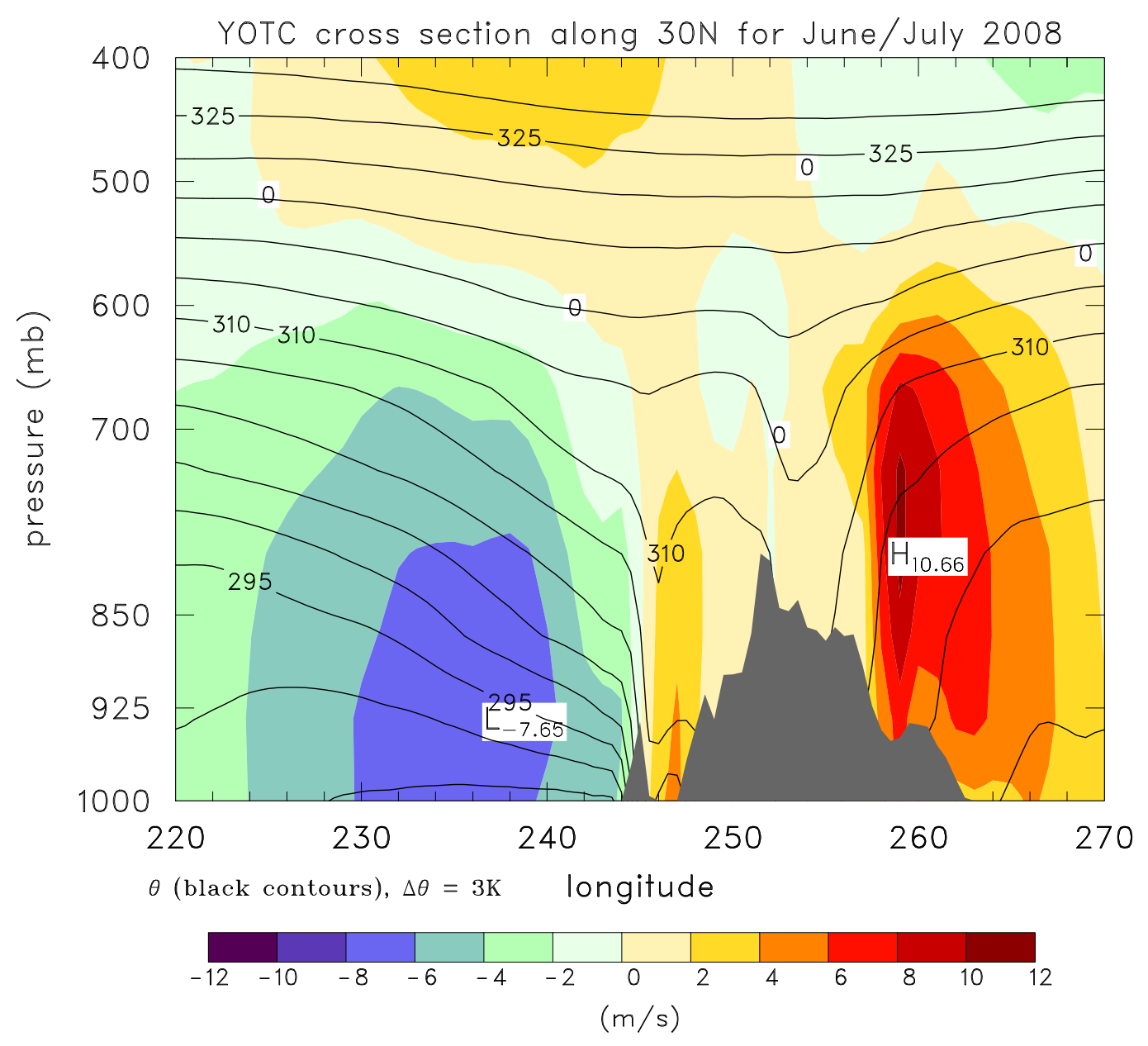

Figure 2.6: Same as 2.4 but for $30 \mathrm{~N}$

and temporal resolution, but because it is from only a single site, the results from Bonner's study is also instructive.

Bonner found that GPLLJs occurred most frequently over Oklahoma and Kansas; this confirms the good choice of location chosen for the study by Whiteman et al. (1997). Bonner also found that GPLLJs tend to have the maximum wind speed occur in the early morning and that they occur most frequently in the months of August and September. In contrast to the theory of Blackadar (1957) he determined that the heights and magnitudes of the LLJs have large variability and do not correlate well with the height of the surface inversion.

The study of Whiteman et al. (1997) revealed several interesting features about the GPLLJ. They did not find a large difference in frequency between the warm and cold seasons. The strongest 


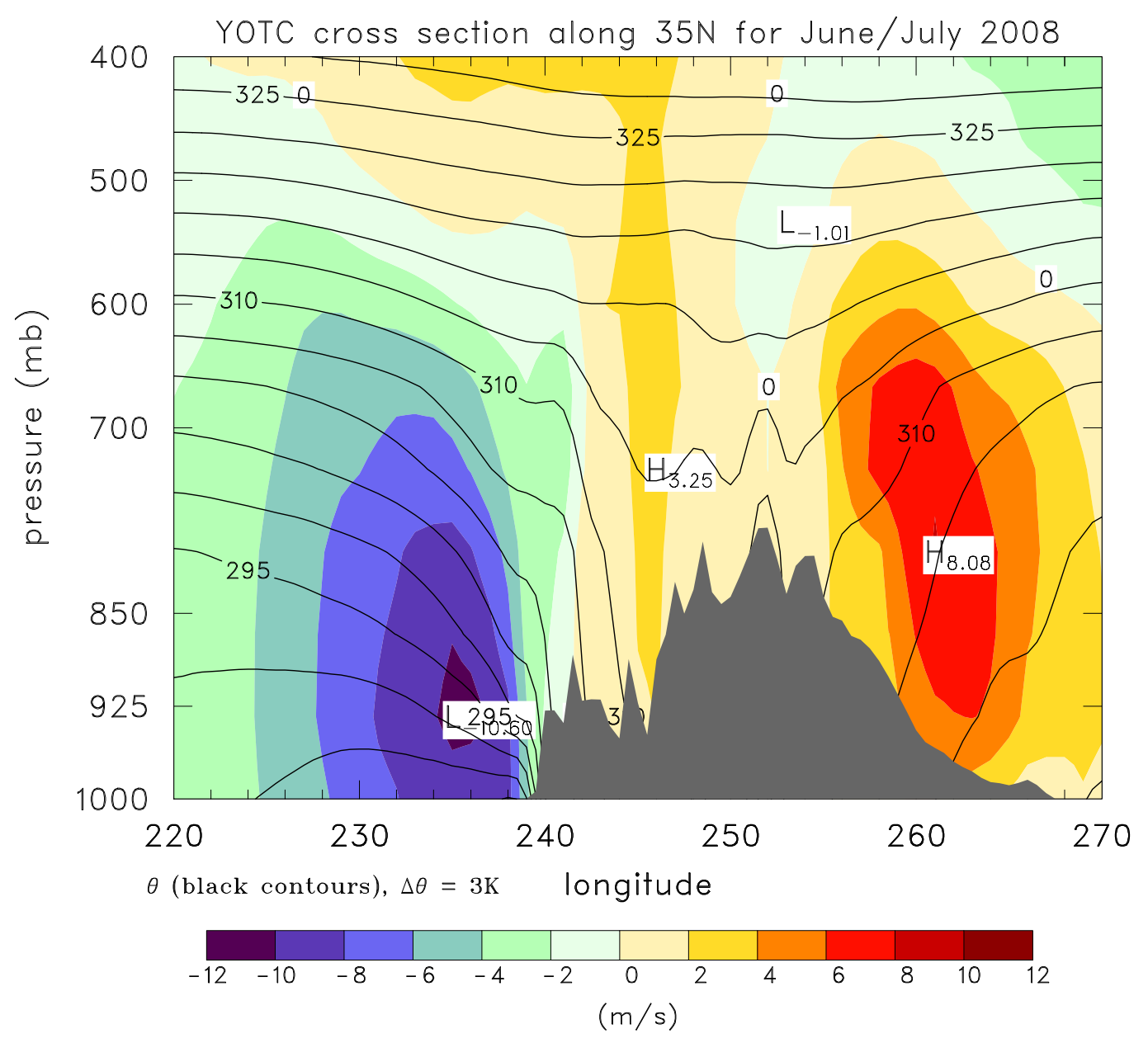

Figure 2.7: Same as 2.4 but for $35 \mathrm{~N}$

LLJs are much more common than previously thought and the mean height of the wind maximum is significantly lower than other studies have reported. The mean height of the wind maximum was given by Bonner (1968) as $785 \mathrm{~m}$ AGL, Mitchell et al. (1995) showed it to be $1000 \mathrm{~m}$ AGL, and Whiteman et al. (1997) reported it to be $596 \mathrm{~m}$ AGL. This discrepancy is claimed to be entirely due to the differences in vertical resolution of the observing tools used in the different studies. It seems clear that there is a significant amount of variability in the height of the wind maxima for both the GPLLJ and the SALLJ and that better vertical resolution is needed in the observations. Based on Whiteman et al. (1997) the vertical structure of the GPLLJ has an average wind speed maximum of 17. $1 \mathrm{~m} / \mathrm{s}$ with this maximum usually occurring below $500 \mathrm{~m}$ AGL. 


\subsubsection{Summary}

The observations presented in this chapter highlight the dominant cyclonic wind field around the Rocky Mountains and the Andes. It should also be noted that the coastal jets of the Americas have common characteristics, as do the LLJs over the plains regions on these two continents. The jets over the plains tend to maximize at night or early in the morning and are often more frequent during the warm season. They have a wind speed maximum on the order of $20 \mathrm{~m} / \mathrm{s}$ and generally occur between $500 \mathrm{~m}$ and $2000 \mathrm{~m}$ AGL. Neither of the coastal jets are as close to the mountains as the plains jets and are more directly tied to the high pressure systems of the Pacific. Likewise, there are similarities between the mountain ranges of the Americas. Both the Rocky Mountains and the Andes are generally oriented in the north-south direction. They both are bordered on the west by the Pacific ocean and on the east by plains gently sloping to lower elevations. On the other hand the Andes are higher, steeper, and narrower than the Rockies and the Andes are much closer to the equator. In the following chapters we explore the hypothesis that these similarities of the LLJs are in part due to the atmospheric response to the heating of these mountains. 


\section{Chapter 3}

\section{DYNAMICAL THEORY OF TOPOGRAPHICALLY BOUND BALANCED MOTIONS ON THE $f$-PLANE}

\subsection{Introduction}

Eliassen (1980) considered the following geostrophic adjustment problem. Initially, a stably stratified fluid is in a state of rest over a level bottom surface on an $f$-plane. Over some arbitrary time interval, the bottom topography is raised to its final shape and any transient inertia-gravity waves are allowed to disperse away. The final adjusted wind and mass fields are then determined via the potential vorticity (PV) invertibility principle. He found that a solution with a constant density lower surface could only occur when the height of the orography was less than some critical value, which is here called the critical crest height. For orography that exceeds the critical crest height, the summit will penetrate into the less dense layers above.

Eliassen derived analytic solutions for the case of an isentropic object with a height less than the critical crest height. He did not plot these solutions or derive solutions for any obstacles that penetrated through the surface density layer (his 'warm' obstacle). His paper clearly demonstrated the usefulness of isopycnic or isentropic coordinates for geostrophic adjustment problems when used in combination with the conservation of potential vorticity.

This research examines a similar problem of the balanced wind and mass fields near topography that has been heated by radiative processes, resulting in a cyclonic flow around the obstacle. This allows for an interpretation of the prominent low-level jet circulations observed over the Americas as part of the topographically bound motion associated with the PV anomaly produced by solar 
heating. Specifically, the Great Plains low-level jet and the California coastal low-level jet are part of the same dynamical response to heating of the Rocky Mountains and both the South American low-level jet and the Chilean coastal low-level jet are part of the same dynamical response to heating of the Andes.

The vertical coordinate of the mathematical model was chosen to be potential temperature because of the simple mathematical form the expression for PV takes, its close analog to Eliassen's model, and the fact that potential temperature generally increases monotonically with height in the atmosphere. This quickly raises the question of how to handle a lower boundary that is not an isentropic surface whenever the domain of interest includes the lower troposphere. An excellent discussion on the implementation of isentropic coordinates is given by Hsu and Arakawa (1990). They list several advantages of using this coordinate system, a few of which are summarized here.

(1) Potential temperature (entropy) is conserved for a material element for adiabatic processes. This implies that isentropic surfaces are also material surfaces.

(2) The expression for Ertel's potential vorticity in isentropic coordinates does not include the vertical derivative of the horizontal velocity. This is one of the reasons isentropic coordinates are especially convenient when analyzing PV dynamics.

(3) This choice of coordinates provides a quasi-Lagrangian view of the general circulation of the atmosphere. When diabatic heating is zero, the pressure torque acting on the coordinate surfaces is the only mechanism for angular momentum to be vertically transferred.

Of course there are disadvantages to using this coordinate system as well. The two primary ones being the already mentioned intersection of isentropes with the lower boundary and the fact that the mass between two surfaces can become infinitesimally small, leading to computational difficulties. As discussed by Hsu and Arakawa (1990) these are technical difficulties rather than intrinsic and can be dealt with in a straightforward way.

To the best of the author's knowledge the idea of using material surfaces for the vertical coordinate was first proposed by Starr (1945). Shortly after this, Lorenz (1955) used isentropic 
surfaces in his definition of available potential energy. He developed the idea of 'massless' layers to cope with the problem of isentropes that intersect the surface. When an isentrope intersects the surface, imagine that it continues just under the surface. The pressure along this subsurface isentrope is defined to be the surface pressure at that location, thus there can be no mass between the isentrope

at the surface and the one 'below' it. This implies the pseudodensity $\left(-\frac{1}{g} \partial p / \partial \theta\right)$ is equal to zero and the PV is infinite in the massless layer. Fulton and Schubert (1991) demonstrated the practicality of using a massless layer with the semigeostrophic equations. They applied this approach to the problem of surface frontogenesis. The basic idea is to formulate the predictive equation in terms of the inverse of the PV. The governing equations are still valid in the massless layer and numerical schemes for solving the system of equations have been presented in the literature (see Fulton and Schubert, 1991; Arakawa and Hsu, 1990).

In the next section the basic elliptic system is set up with appropriate boundary conditions and the invertibility principle is derived. All of the interesting physics of the problem comes directly from the lower boundary condition and the intersection of isentropic surfaces with this boundary. For the particular system studied here, finding an analytic solution is much simpler in spectral space than it is in physical space. With this in mind a horizontal Fourier transform is applied to the system and results in a vertical structure equation. This vertical structure equation can be solved in at least two ways. The method using a Green's function is presented because of its compactness and insight.

\subsection{Basic governing equations}

The following sections present equations describing the simplest possible physical system that still retains the ability to describe the balanced response of a fluid to underlying heated orography. Consider the inviscid, adiabatic, quasi-geostrophic, quasi-hydrostatic, motions of a compressible stratified fluid on an $f$-plane. The basic equations for this system can be written as

$$
\begin{aligned}
& \frac{D u_{g}}{D t}-f v+\frac{\partial M}{\partial x}=0 \\
& \frac{D v_{g}}{D t}+f u+\frac{\partial M}{\partial y}=0
\end{aligned}
$$




$$
\begin{gathered}
\frac{\partial M}{\partial \theta}=\Pi \\
\frac{D \sigma}{D t}+\sigma\left(\frac{\partial u}{\partial x}+\frac{\partial v}{\partial y}\right)=0
\end{gathered}
$$

where

$$
\left(f v_{g},-f u_{g}\right)=\left(\frac{\partial M}{\partial x}, \frac{\partial M}{\partial y}\right)
$$

are the geostrophic velocity components. The Exner function is defined by $\Pi=c_{p}\left(p / p_{0}\right)^{R / c_{p}}$, $M=\theta \Pi+\phi$ is the Montgomery potential, $\phi$ is the geopotential, $\sigma=-(1 / g)(\partial p / \partial \theta)$ is the pseudo-density, and

$$
\frac{D}{D t}=\frac{\partial}{\partial t}+u \frac{\partial}{\partial x}+v \frac{\partial}{\partial y}
$$

is the material derivative. The constant reference pressure is assumed to be $p_{0}=1000 \mathrm{hPa}, R=$ $287.0 \mathrm{~J} \mathrm{~kg}^{-1} \mathrm{~K}^{-1}$ is the gas constant of dry air, and $c_{p}=1004 \mathrm{~J} \mathrm{~kg}^{-1} \mathrm{~K}^{-1}$ is the specific heat of dry air at constant pressure. The buoyancy frequency $N(\theta)$ is an important parameter that gives a measure for the degree of stability of the fluid under consideration. It is here defined by

$$
N^{2}(\theta)=\frac{g^{2}}{\theta^{2}}\left(-\frac{d \tilde{\Pi}}{d \theta}\right)^{-1} .
$$

When $N^{2}>0$ the fluid is stable, when $N^{2}<0$ the fluid is unstable. Following Eliassen (1980) two special cases of the buoyancy frequency are considered which will be referred to as reference state 1 and reference state 2 . For Reference state 1 we assume that the buoyancy frequency is inversely proportional to $\theta$, i.e., $N(\theta)=N_{1} \theta_{B} / \theta$, where $N_{1}$ and $\theta_{B}$ are constants. A second reference state could also be defined by assuming the buoyancy frequency is a constant, i.e., $N(\theta)=N_{2}$.

\subsection{Invertibility principle}

The material conservation law for potential vorticity takes the form $D P / D t=0$. For $y$ independent motions the potential vorticity is defined by

$$
P=\left(f+\frac{\partial v}{\partial x}\right)\left(-\frac{1}{g} \frac{\partial p}{\partial \theta}\right)^{-1},
$$

with $f$ denoting the constant Coriolis parameter, $g$ the acceleration of gravity, $v(x, \theta)$ the meridional component of the flow, and $p(x, \theta)$ the pressure. In the far-field the flow vanishes, and the 
pressure and potential vorticity take on the horizontally homogeneous values $\tilde{p}(\theta)$ and $\tilde{P}(\theta)$, which are related by

$$
\tilde{P}=f\left(-\frac{1}{g} \frac{\partial \tilde{p}}{\partial \theta}\right)^{-1}
$$

Denoting the density by $\rho(x, \theta)$ and the far-field density by $\tilde{\rho}(\theta)$, it is easily shown that $\theta \rho(d \Pi / d p)=1$ and $\theta \tilde{\rho}(d \tilde{\Pi} / d \tilde{p})=1$, where $\tilde{\Pi}=c_{p}\left(\tilde{p} / p_{0}\right)^{R / c_{p}}$. This allows the ratio of (3.8) to (3.9) to be written in the form

$$
\begin{aligned}
f \frac{P}{\tilde{P}} & =\left(f+\frac{\partial v}{\partial x}\right)\left(\frac{\partial \tilde{p} / \partial \theta}{\partial p / \partial \theta}\right) \\
& =\left(f+\frac{\partial v}{\partial x}\right)\left(\frac{\tilde{\rho}(d \tilde{\Pi} / d \tilde{p})(\partial \tilde{p} / \partial \theta)}{\rho(d \Pi / d p)(\partial p / \partial \theta)}\right) \\
& =\left(f+\frac{\partial v}{\partial x}\right)\left(\frac{\partial \tilde{\Pi} / \partial \theta}{\partial \Pi / \partial \theta}\right),
\end{aligned}
$$

where the last equality follows from approximating the $(\tilde{\rho} / \rho)$ factor by unity. Using the definition for buoyancy frequency, (3.10) can also be written in the form

$$
\frac{\partial v}{\partial x}+\left(\frac{f \theta^{2} N^{2} P}{g^{2} \tilde{P}}\right) \frac{\partial \Pi^{\prime}}{\partial \theta}=f\left(\frac{P}{\tilde{P}}-1\right),
$$

where $\Pi^{\prime}(x, \theta)=\Pi(x, \theta)-\tilde{\Pi}(\theta)$ is the Exner function anomaly.

We shall require that (3.11) holds in a region that includes an underlying topographic feature whose geopotential surface is specified by $\phi_{S}(x)$. We assume that, due to radiative processes, the potential temperature varies along the topography according to the specified function $\theta_{S}(x)$. For simplicity, we assume that both $\phi_{S}(x)$ and $\theta_{S}(x)$ are symmetric about $x=0$. The inverse of $\theta_{S}(x)$ is $x_{S}(\theta)$. When a $\theta$ surface intersects the topographic surface, it is considered to run just under the topographic surface with a pressure equal to the surface pressure and thus form a layer that contains no mass. The point at which a particular $\theta$ surface intersects the topographic surface is defined to be $x_{S}(\theta)$. The isentropic surface that is below the earth's surface over the topographic feature and is at the earth's surface in the far-field is labeled $\theta_{B}$. The $\theta_{B}$ surface is the lowest isentrope that is in the massless layer for all values of $x$ and defines the lower boundary of the domain. Thus, in $\theta$ space, the massless layer is defined to be the region $\theta_{B} \leq \theta<\theta_{S}(x)$. The crest isentrope $\left(\theta_{C}\right)$ is defined to be the isentrope that just touches the crest of the ridge. Assuming both geostrophic and 
hydrostatic balance leads to the thermal wind relation given below in (3.13). Equations (3.12) and (3.13) are Cauchy-Riemann conditions on the unknown functions $v(x, \theta)$ and $\Pi^{\prime}(x, \theta)$.

We now assume that, except in the massless layer, the potential vorticity is uniform on each isentropic surface, i.e., $P(x, \theta)=\tilde{P}(\theta)$ for $x_{S}(\theta)<x<\infty$, so that the right hand side of (3.11) is zero and (3.11) simplifies to the top entry in (3.12) below. In the massless layer, the pseudodensity vanishes, i.e., $\partial p / \partial \theta=0$, or equivalently $\partial \Pi / \partial \theta=0$, which can be written as the second line in (3.12). An alternative derivation consists of multiplying (3.11) by $\tilde{P} / P$ and then taking the limit as $P \rightarrow \infty$. As for boundary conditions, we require that $v$ and $\Pi^{\prime}$ approach zero in the farfield. We also require that the upper boundary is both an isentropic $\left(\theta=\theta_{T}\right)$ and isobaric surface, which is expressed in the second line of (3.14). To formulate the lower boundary condition we combine the $x$-derivative of $M-\theta \Pi=\phi$ with the geostrophic and hydrostatic relations to obtain $f[v-\theta(\partial v / \partial \theta)]=(\partial \phi / \partial x)$, which, when applied at $\theta=\theta_{B}$ yields the bottom entry in (3.14). In summary, the elliptic problem is

$$
\begin{gathered}
\frac{\partial v}{\partial x}+\left(\frac{f \theta^{2} N^{2}}{g^{2}}\right) \frac{\partial \Pi^{\prime}}{\partial \theta}=0 \text { for } x_{S}(\theta)<x<\infty \\
\left(\frac{f \theta^{2} N^{2}}{g^{2}}\right) \frac{\partial \Pi^{\prime}}{\partial \theta}=f \text { for } 0 \leq x<x_{S}(\theta) \\
f \frac{\partial v}{\partial \theta}-\frac{\partial \Pi^{\prime}}{\partial x}=0 \text { for } 0 \leq x<\infty, \quad \theta_{B} \leq \theta \leq \theta_{T}
\end{gathered}
$$

with boundary conditions

$$
\begin{gathered}
v \rightarrow 0 \text { and } \Pi^{\prime} \rightarrow 0 \text { as } x \rightarrow \infty, \\
\Pi^{\prime}=0 \text { at } \theta=\theta_{T}, \\
f\left(v-\theta \frac{\partial v}{\partial \theta}\right)=\frac{d \phi_{S}(x)}{d x} \text { at } \theta=\theta_{B} .
\end{gathered}
$$

\subsection{Fourier transform of the invertibility principle}

To solve the invertibility problem (3.12)-(3.14) Fourier integral transforms are used. For simplicity it is assumed that the specified functions $\phi_{S}(x)$ and $\theta_{S}(x)$ are symmetric in $x$, so that $\Pi^{\prime}(x, \theta)$ is symmetric in $x$ and $v(x, \theta)$ is antisymmetric in $x$. The Fourier sine transform pair for 
$v(x, \theta)$ is

$$
\begin{gathered}
\hat{v}(k, \theta)=\frac{2}{\pi} \int_{0}^{\infty} v(x, \theta) \sin (k x) d x, \\
v(x, \theta)=\int_{0}^{\infty} \hat{v}(k, \theta) \sin (k x) d k,
\end{gathered}
$$

while the Fourier cosine transform pair for $\Pi^{\prime}(x, \theta)$ is

$$
\begin{aligned}
\hat{\Pi}^{\prime}(k, \theta) & =\frac{2}{\pi} \int_{0}^{\infty} \Pi^{\prime}(x, \theta) \cos (k x) d x, \\
\Pi^{\prime}(x, \theta) & =\int_{0}^{\infty} \hat{\Pi}^{\prime}(k, \theta) \cos (k x) d k .
\end{aligned}
$$

A similar cosine transform pair exists for the surface geopotential $\phi_{S}(x)$ and its transform $\hat{\phi}_{S}(k)$.

We now wish to Fourier transform (3.12), (3.13), and the last two lines of (3.14). To Fourier transform (3.13) we multiply it by $\sin (k x)$ and integrate over $x$ from 0 to $\infty$, thereby obtaining (3.23) below. To Fourier transform (3.12) we first multiply the top line in (3.12) by $\cos (k x)$ and integrate over $x$ from $x_{S}(\theta)$ to $\infty$, thereby obtaining

$$
\begin{aligned}
\int_{0}^{\infty} \frac{\partial v(x, \theta)}{\partial x} \cos (k x) d x & -\int_{0}^{x_{S}(\theta)} \frac{\partial v(x, \theta)}{\partial x} \cos (k x) d x+\frac{f \theta^{2} N^{2}}{g^{2}} \frac{d}{d \theta} \int_{x_{S}(\theta)}^{\infty} \Pi^{\prime}(x, \theta) \cos (k x) d x \\
& =-\frac{f \theta^{2} N^{2}}{g^{2}} \Pi^{\prime}\left(x_{S}(\theta), \theta\right) \frac{d x_{S}(\theta)}{d \theta} \cos \left(k x_{S}(\theta)\right) .
\end{aligned}
$$

Similarly, multiplying the bottom line in (3.12) by $\cos (k x)$ and integrating over $x$ from 0 to $x_{S}(\theta)$, we obtain

$$
\begin{gathered}
\frac{f \theta^{2} N^{2}}{g^{2}} \frac{d}{d \theta} \int_{0}^{x_{S}(\theta)} \Pi^{\prime}(x, \theta) \cos (k x) d x= \\
\int_{0}^{x_{S}(\theta)} f \cos (k x) d x+\frac{f \theta^{2} N^{2}}{g^{2}} \Pi^{\prime}\left(x_{S}(\theta), \theta\right) \frac{d x_{S}(\theta)}{d \theta} \cos \left(k x_{S}(\theta)\right) .
\end{gathered}
$$

Taking the sum of (3.19) and (3.20), noting the cancellation of the two $\Pi^{\prime}\left(x_{S}(\theta), \theta\right)$ terms, we obtain

$$
\begin{gathered}
\int_{0}^{\infty} \frac{\partial v(x, \theta)}{\partial x} \cos (k x) d x+\frac{f \theta^{2} N^{2}}{g^{2}} \frac{d}{d \theta} \int_{0}^{\infty} \Pi^{\prime}(x, \theta) \cos (k x) d x= \\
\int_{0}^{x_{S}(\theta)}\left(f+\frac{\partial v(x, \theta)}{\partial x}\right) \cos (k x) d x
\end{gathered}
$$

Integrating the first term by parts, using the symmetry condition $v(0, \theta)=0$ and the lateral boundary condition $v \rightarrow 0$ as $x \rightarrow \infty$, and then noting the transform relations (3.15) and (3.17), we obtain 
(3.22), where $F(k, \theta)$ is defined in (3.25). In summary, the Fourier transform of the elliptic problem (3.12)-(3.13) is

$$
\begin{gathered}
k \hat{v}+\left(\frac{f \theta^{2} N^{2}}{g^{2}}\right) \frac{d \hat{\Pi}^{\prime}}{d \theta}=F(k, \theta), \\
f \frac{d \hat{v}}{d \theta}+k \hat{\Pi}^{\prime}=0
\end{gathered}
$$

with boundary conditions

$$
\begin{aligned}
\hat{\Pi}^{\prime} & =0 \text { at } \theta=\theta_{T}, \\
\theta \frac{d \hat{v}}{d \theta}-\hat{v} & =\frac{k}{f} \hat{\phi}_{S}(k) \text { at } \theta=\theta_{B},
\end{aligned}
$$

where $F(k, \theta)$ is defined by

$$
\begin{aligned}
& F(k, \theta)=\frac{2}{\pi} \int_{0}^{x_{S}(\theta)}\left(f+\frac{\partial v}{\partial x}\right) \cos (k x) d x \\
& =\frac{2}{\pi} \int_{0}^{x_{S}(\theta)}\left[f+\frac{1}{f}\left(\theta \frac{d^{2} \Pi_{S}(x)}{d x^{2}}+\frac{d^{2} \phi_{S}(x)}{d x^{2}}\right)\right] \cos (k x) d x .
\end{aligned}
$$

The first line of (3.25) can be interpreted as a measure of the "absolute isentropic vorticity in the massless layer." In the second line $F(k, \theta)$ is composed of three separate pieces, the first is due to the Coriolis force, the second depends on the radiative heating along the surface, and the third piece depends on the particular shape of the ridge being studied. Note that, for $\theta>\theta_{C}$, (3.25) yields $F(k, \theta)=0$ since $x_{S}(\theta)=0$. In other words, (3.22) is a homogeneous equation above the crest isentrope. The second line in (3.25) is derived as follows. In the massless layer $\phi(x, \theta)=\phi_{S}(x)$ and $\Pi(x, \theta)=\Pi\left(x, \theta_{S}(x)\right)$, so that the Montgomery potential in the massless layer is given by $M(x, \theta)=\theta \Pi\left(\theta_{S}(x)\right)+\phi_{S}(x)$. Using this formula for $M(x, \theta)$ in $(\partial v / \partial x)=(1 / f)\left(\partial^{2} M / \partial x^{2}\right)$, we obtain the second line in (3.25), which allows us to compute $F(k, \theta)$ in terms of the specified functions $\Pi_{S}(x), \theta_{S}(x)$, and $\phi_{S}(x)$. Rather than having to specify both $\Pi_{S}(x)$ and $\theta_{S}(x)$ it would be nice if $\theta_{S}(x)$ was the only function (except the lower geopotential surface) that needed to be specified. The Exner function at the geopotential surface can be written as

$$
\Pi_{S}\left(p_{S}(x)\right)=c_{p}\left(\frac{p_{s}(x)}{p_{0}}\right)^{\gamma}=c_{p}\left(\frac{\tilde{\rho} \theta_{S} R}{p_{0}}\right)^{\frac{\gamma}{1-\gamma}} .
$$

Where $\gamma=R / c_{p}$ and the assumption $\tilde{\rho} / \rho=1$ has been used, as it was in the derivation of (3.11). Now, rather than having to specify both $\theta_{S}(x)$ and $p_{s}(x)$ we only need $\theta_{S}(x)$. For a given topo- 
graphic scenario, once $\theta_{S}(x)$ is given, the wind and mass fields that result from the adjustment to the topography and temperature along the lower boundary should be computable.

As can be seen from the vertical structure equations (3.22)-(3.24), balanced wind and mass fields are forced in two ways: by $F(k, \theta)$ and by $\hat{\phi}_{S}(k)$. When both $F(k, \theta)$ and $\hat{\phi}_{S}(k)$ vanish, the solutions of (3.22)-(3.24) are $\hat{v}(k, \theta)=0$ and $\hat{\Pi}^{\prime}(k, \theta)=0$. Thus, for nontrivial solutions we can consider three special cases: (i) nonzero topography $\left(\hat{\phi}_{S}(k) \neq 0\right)$ with no variation of potential temperature along the bottom boundary $(F(k, \theta)=0)$; (ii) flat topography $\left(\hat{\phi}_{S}(k)=0\right)$ but with variation of potential temperature along the bottom boundary $(F(k, \theta) \neq 0)$; and (iii) nonzero topography with a variation of potential temperature along the surface. Eliassen (1980) studied case (i) and showed that physically reasonable solutions are possible only if the crest height of the mountain is less than a critical value. In case (i) the topographically bound balanced flow is anticyclonic, so this special case is not useful for describing cyclonic flows such as those shown in Figs. 1.2 and 1.3.

Our analysis shows that, for a given reference state buoyancy frequency profile $N(\theta)$ and topography $\phi_{S}(x)$, the only remaining field that needs to be specified is the potential temperature

distribution along the lower boundary, i.e., $\theta_{S}(x)$ or its inverse $x_{S}(\theta)$. The entire distributions of the balanced wind and mass fields are then given by the solutions $v(x, \theta)$ and $\Pi^{\prime}(x, \theta)$ of the elliptic problem (3.12)-(3.14).

In the next section we consider the $N(\theta)$ profile for reference state 1 which allows for a simple analytical solution of (3.22)-(3.24).

\subsection{Solution of the vertical structure equation}

The Exner function for reference state 1 can be found by integrating (3.7) to obtain

$$
\tilde{\Pi}_{1}(\theta)=c_{p}-\frac{g^{2}}{\theta_{B}^{2} N_{1}^{2}}\left(\theta-\theta_{B}\right)
$$

after which the reference state hydrostatic equation can be integrated to obtain

$$
\tilde{M}_{1}(\theta)=c_{p} \theta-\frac{g^{2}}{2 \theta_{B}^{2} N_{1}^{2}}\left(\theta-\theta_{B}\right)^{2}
$$




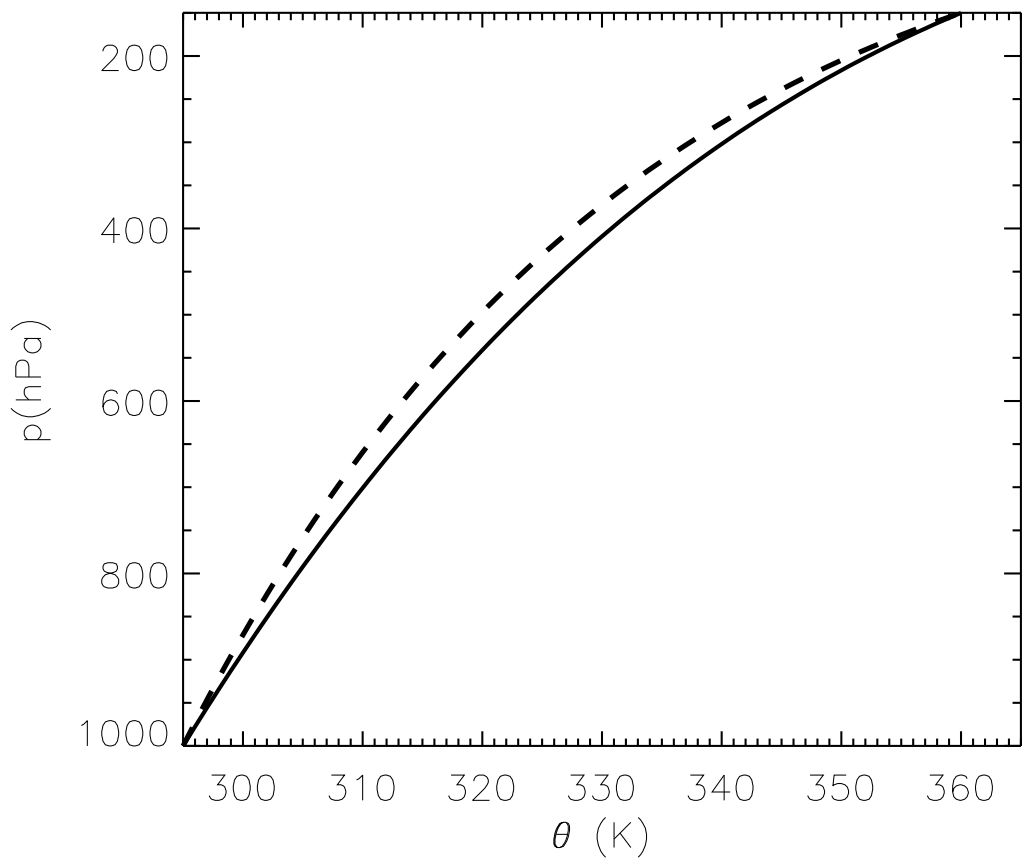

Figure 3.1: Pressure as a function of $\theta$ as computed from (3.27) for reference state 1 (solid curve) and for reference state 2 (dashed curve).

Since $\tilde{\phi}_{1}(\theta)=\tilde{M}_{1}(\theta)-\theta \tilde{\Pi}_{1}(\theta)$, we can use (3.27) and (3.28) to obtain

$$
\tilde{\phi}_{1}(\theta)=\frac{g^{2}}{2 \theta_{B}^{2} N_{1}^{2}}\left(\theta^{2}-\theta_{B}^{2}\right)
$$

The relationship of pressure and potential temperature for reference state 1 , as determined from (3.27), is plotted as the solid curve in Fig. 3.1 and for reference state 2 as the dashed curve, where we have chosen $c_{p}=1004.5 \mathrm{~J} \mathrm{~kg}^{-1} \mathrm{~K}^{-1}, g=9.8 \mathrm{~m} \mathrm{~s}^{-2}, \theta_{B}=295 \mathrm{~K}$, and $\left(\theta_{B} / g\right) N_{1}^{2}=5.1373 \mathrm{~K}$ $\mathrm{km}^{-1}$.

Equations (3.22)-(3.23) represent a system of two equations and two unknowns. When one of the unknowns is eliminated a second order ordinary differential equation with variable coefficients is obtained. If $\hat{\Pi}^{\prime}(k, \theta)$ is eliminated and reference state 1 is assumed, the second order ordinary differential equation with constant coefficients for $\hat{v}(k, \theta)$ is found to be

$$
\frac{d^{2} \hat{v}}{d \theta^{2}}-\kappa^{2} \hat{v}=-\frac{g \kappa}{f \theta_{B} N_{1}} F
$$

and the boundary conditions are

$$
\frac{d \hat{v}}{d \theta}=0 \text { at } \theta=\theta_{T}
$$




$$
\theta \frac{d \hat{v}}{d \theta}-\hat{v}=\frac{k}{f} \hat{\phi}_{S}(k) \text { at } \theta=\theta_{B}
$$

where $\kappa(k)=g k /\left(f \theta_{B} N_{1}\right)$. We now solve (3.30)-(3.32) via the Green's function $\mathcal{G}\left(k, \theta, \theta^{\prime}\right)$.

Green's functions are quite useful when solving nonhomogeneous differential equations. The basic approach is to replace the forcing function with a delta function and solve the resulting equation. Thus from (3.30) the Green's function is the solution of the differential equation

$$
\frac{d^{2} \mathcal{G}}{d \theta^{2}}-\kappa^{2} \mathcal{G}=-\kappa \delta\left(\theta-\theta^{\prime}\right)
$$

with the homogeneous boundary conditions

$$
\begin{gathered}
\frac{d \mathcal{G}}{d \theta}=0 \text { at } \theta=\theta_{T}, \\
\theta \frac{d \mathcal{G}}{d \theta}-\mathcal{G}=0 \text { at } \theta=\theta_{B},
\end{gathered}
$$

where the Dirac delta function $\delta\left(\theta-\theta^{\prime}\right)$ vanishes for $\theta \neq \theta^{\prime}$ and satisfies

$$
\int_{\theta^{\prime}-}^{\theta^{\prime}+} \delta\left(\theta-\theta^{\prime}\right) d \theta=1 .
$$

The variable $\theta^{\prime}$ represents a single point of excitation; or an impulse at the point in the domain where $\theta=\theta^{\prime}$. Thus $\mathcal{G}$ is the solution of (3.30) when the forcing is concentrated at the point $\theta=\theta^{\prime}$. The solution of (3.30) for general forcing is then given by an integral over multiple Green's functions at each point of excitation.

For $\theta^{\prime} \leq \theta \leq \theta_{T}$, the Green's function is a constant times the solution of the homogeneous version of (3.30) satisfying the upper boundary condition (3.31), while for $\theta_{B} \leq \theta \leq \theta^{\prime}$ the Green's function is a constant times the solution of the homogeneous version of (3.30) satisfying the lower boundary condition (3.32). The two constants are determined by requiring that $\mathcal{G}\left(k, \theta, \theta^{\prime}\right)$ is continuous at $\theta=\theta^{\prime}$ and that the jump in the first derivative satisfies

$$
\left[\frac{d \mathcal{G}}{d \theta}\right]_{\theta^{\prime}-}^{\theta^{\prime}+}=-\kappa,
$$

which is obtained by integrating (3.33) across a narrow region surrounding $\theta=\theta^{\prime}$, making use of 


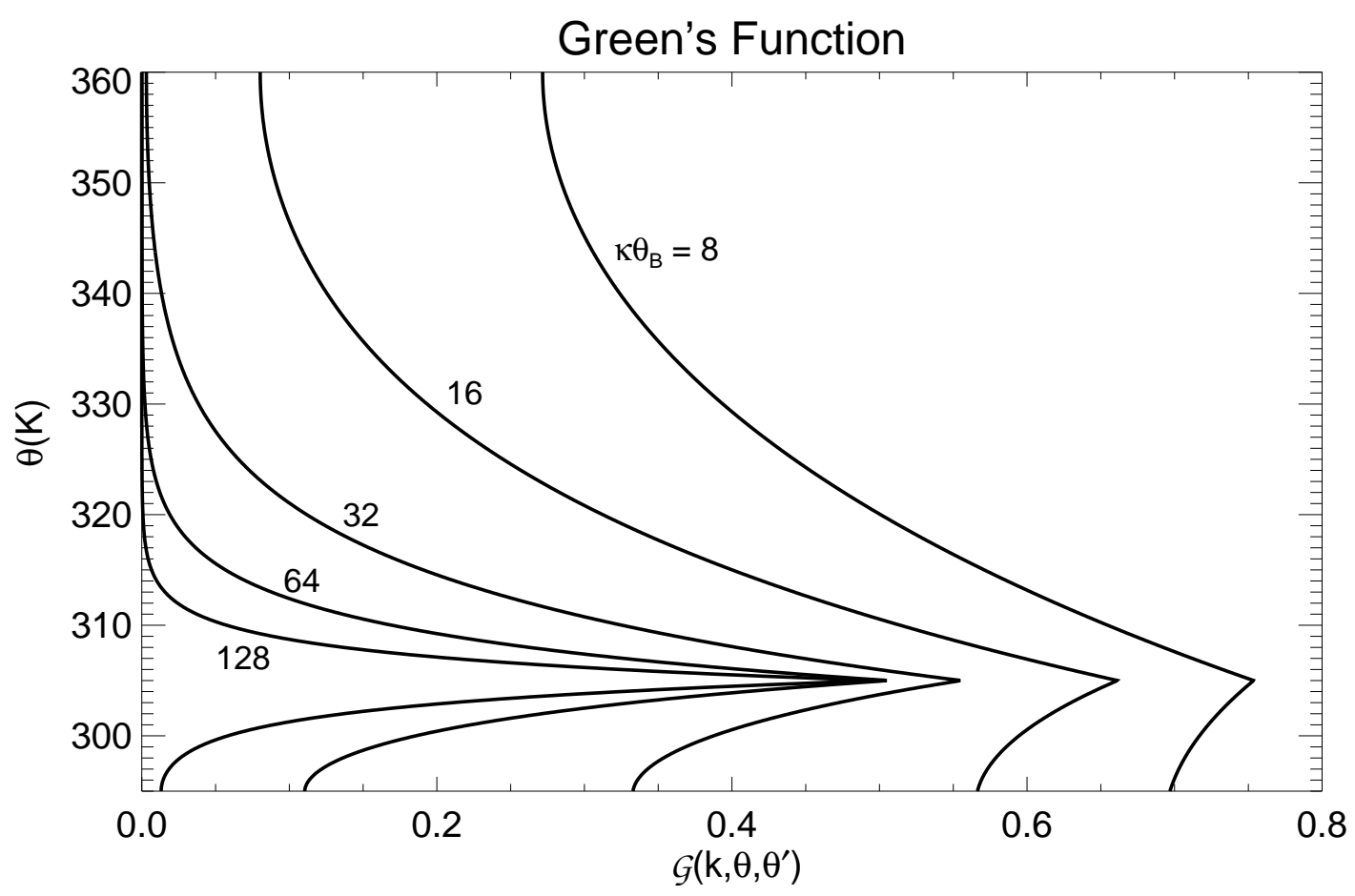

Figure 3.2: The Green's function for select values of $\kappa \theta_{B}$. For this figure $\theta^{\prime}=305 \mathrm{~K}$.

(3.36). This procedure results in

$\mathcal{G}\left(k, \theta, \theta^{\prime}\right)=\frac{1}{D} \begin{cases}\cosh \left[\kappa\left(\theta_{T}-\theta\right)\right]\left\{\kappa \theta_{B} \cosh \left[\kappa\left(\theta^{\prime}-\theta_{B}\right)\right]+\sinh \left[\kappa\left(\theta^{\prime}-\theta_{B}\right)\right]\right\} & \text { for } \theta^{\prime} \leq \theta \leq \theta_{T}, \\ \cosh \left[\kappa\left(\theta_{T}-\theta^{\prime}\right)\right]\left\{\kappa \theta_{B} \cosh \left[\kappa\left(\theta-\theta_{B}\right)\right]+\sinh \left[\kappa\left(\theta-\theta_{B}\right)\right]\right\} & \text { for } \theta_{B} \leq \theta \leq \theta^{\prime},\end{cases}$

where

$$
D=\cosh \left[\kappa\left(\theta_{T}-\theta_{B}\right)\right]+\kappa \theta_{B} \sinh \left[\kappa\left(\theta_{T}-\theta_{B}\right)\right] .
$$

To express the solution $\hat{v}(k, \theta)$ in terms of the Green's function, we multiply (3.30) by $\mathcal{G}\left(k, \theta, \theta^{\prime}\right)$, multiply (3.33) by $\hat{v}(k, \theta)$, and then take the difference of the resulting equations to obtain

$$
\frac{d}{d \theta}\left(\mathcal{G} \frac{d \hat{v}}{d \theta}-\hat{v} \frac{d \mathcal{G}}{d \theta}\right)=-\frac{g \kappa}{f \theta_{B} N_{1}} F \mathcal{G}+\kappa \hat{v} \delta\left(\theta-\theta^{\prime}\right)
$$

Integrating (3.40) over $\theta$, using the top boundary conditions (3.31) and (3.35) and the bottom boundary conditions (3.32) and (3.35), using the delta function property (3.36), and finally using the 
Green's function symmetry property $\mathcal{G}\left(k, \theta^{\prime}, \theta\right)=\mathcal{G}\left(k, \theta, \theta^{\prime}\right)$, we obtain

$$
\begin{aligned}
& \hat{v}(k, \theta)=\frac{g}{f \theta_{B} N_{1}} \int_{\theta_{B}}^{\theta_{T}} F\left(k, \theta^{\prime}\right) \mathcal{G}\left(k, \theta, \theta^{\prime}\right) d \theta^{\prime} \\
& -\frac{N_{1}}{g} \hat{\phi}_{S}(k)\left(\frac{\cosh \left[\kappa\left(\theta_{T}-\theta\right)\right]}{\cosh \left[\kappa\left(\theta_{T}-\theta_{B}\right)\right]+\kappa \theta_{B} \sinh \left[\kappa\left(\theta_{T}-\theta_{B}\right)\right]}\right) .
\end{aligned}
$$

Using $\hat{v}$ in combination with (3.23) gives

$$
\begin{aligned}
& \hat{\Pi}^{\prime}(k, \theta)=-\frac{g^{2}}{f \theta_{B}^{2} N_{1}^{2}} \int_{\theta_{B}}^{\theta_{T}} F\left(k, \theta^{\prime}\right) \frac{\partial \mathcal{G}\left(k, \theta, \theta^{\prime}\right)}{\partial \theta} d \theta^{\prime} \\
& \quad-\hat{\phi}_{S}(k)\left(\frac{\kappa \sinh \left[\kappa\left(\theta_{T}-\theta\right)\right]}{\cosh \left[\kappa\left(\theta_{T}-\theta_{B}\right)\right]+\kappa \theta_{B} \sinh \left[\kappa\left(\theta_{T}-\theta_{B}\right)\right]}\right) .
\end{aligned}
$$

The first term on the right hand side of the these solutions describes the response of the fluid due to the isentropic vorticity forcing in the massless layer, the second term describes the response due to the orography.

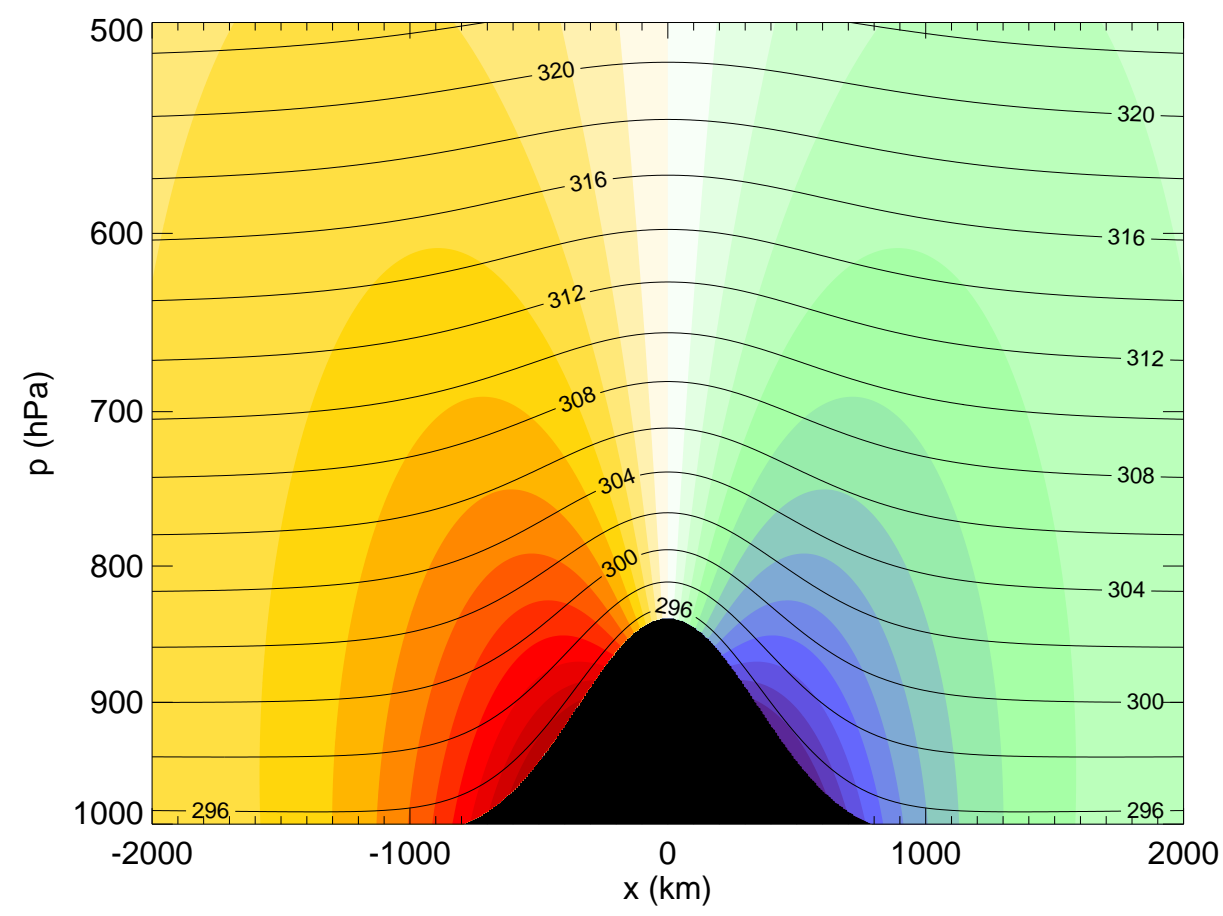

Figure 3.3: Wind field for an isentropic lower boundary. The Gaussian halfwidth of the mountain is $500 \mathrm{~km}$, the maximum height is $\mathrm{H}=1700 \mathrm{~m}$, and the latitude is $30 \mathrm{~N}$. Solid black contours are the isentropes.

Figure 3.3 shows the solution for an isentropic mountain. There is no massless layer in this case so the entire solution is forced from the term in equation (3.41) that involves the geopotential. 
Note that although this chapter has been developed using $\theta$ as the vertical coordinate, and both $\hat{v}$ and $\hat{\Pi}$ were computed in $(x, \theta)$-space this figure is shown in $(x, p)$-space. This makes it easier to view the isentropic mountain that is forcing the atmospheric response. Figures will be shown in Chapter 5 using both $(x, \theta)$-space and $(x, \theta)$-space and the relative advantages will be discussed.

\subsection{Critical crest height}

An important parameter of the preceding theory is the maximum height an obstacle can have while remaining an isentropic object with no isentropes intersecting its surface. In the following an analytical expression is derived which predicts this height. Although the details are different, the logic of this section closely follows that of Eliassen (1980) in his derivation of the critical crest height $\left(H_{c}\right)$.

For our balanced physical system we have assumed that hydrostatic balance $(\partial M / \partial \theta=\Pi)$

holds. Because pressure decreases with height, it follows that $\Pi$ will also decrease with height and $\theta$ must increase monotonically with height for it to qualify as a vertical coordinate. This implies

$$
\frac{\partial^{2} M}{\partial \theta^{2}}=\frac{\partial \Pi}{\partial \theta}<0
$$

Assuming the Exner function can be written as a sum of a mean part and a perturbation part and using the buoyancy frequency from reference state 1 , the above inequality can be written as

$$
\left(\frac{g}{N_{1} \theta_{B}}\right)^{2}>\frac{\partial \Pi^{\prime}}{\partial \theta}=\int_{0}^{\infty} \frac{\partial \hat{\Pi}^{\prime}}{\partial \theta} \cos (k x) d k .
$$

The solution for an isentropic mountain was found to be

$$
\frac{\theta_{B} N_{1}}{g} \hat{\Pi}^{\prime}(k, \theta)=-\frac{k}{f} \hat{\phi}_{S}(k)\left(\frac{e^{-\kappa\left(\theta-\theta_{B}\right)}-e^{-\kappa\left(2 \theta_{T}-\theta_{B}-\theta\right)}}{1+\kappa \theta_{B}+\left(1-\kappa \theta_{B}\right) e^{-2 \kappa\left(\theta_{T}-\theta_{B}\right)}}\right) .
$$

We now can write a general inequality that depends on the function specifying topography which must hold for a hydrostatic atmosphere:

$$
\left(\frac{g}{N_{1} \theta_{B}}\right)^{2}>\int_{0}^{\infty} \kappa^{2} \hat{\phi}_{S}(k)\left(\frac{e^{-\kappa\left(\theta-\theta_{B}\right)}+e^{-\kappa\left(2 \theta_{T}-\theta_{B}-\theta\right)}}{1+\kappa \theta_{B}+\left(1-\kappa \theta_{B}\right) e^{-2 \kappa\left(\theta_{T}-\theta_{B}\right)}}\right) \cos (k x) d k .
$$

For an isentropic surface, there is no massless layer and the potential temperature along the orography will be constant $\left(\theta_{S}=\theta_{B}=\right.$ constant $)$. The maximum height of the obstacle will be at $x=0$ 


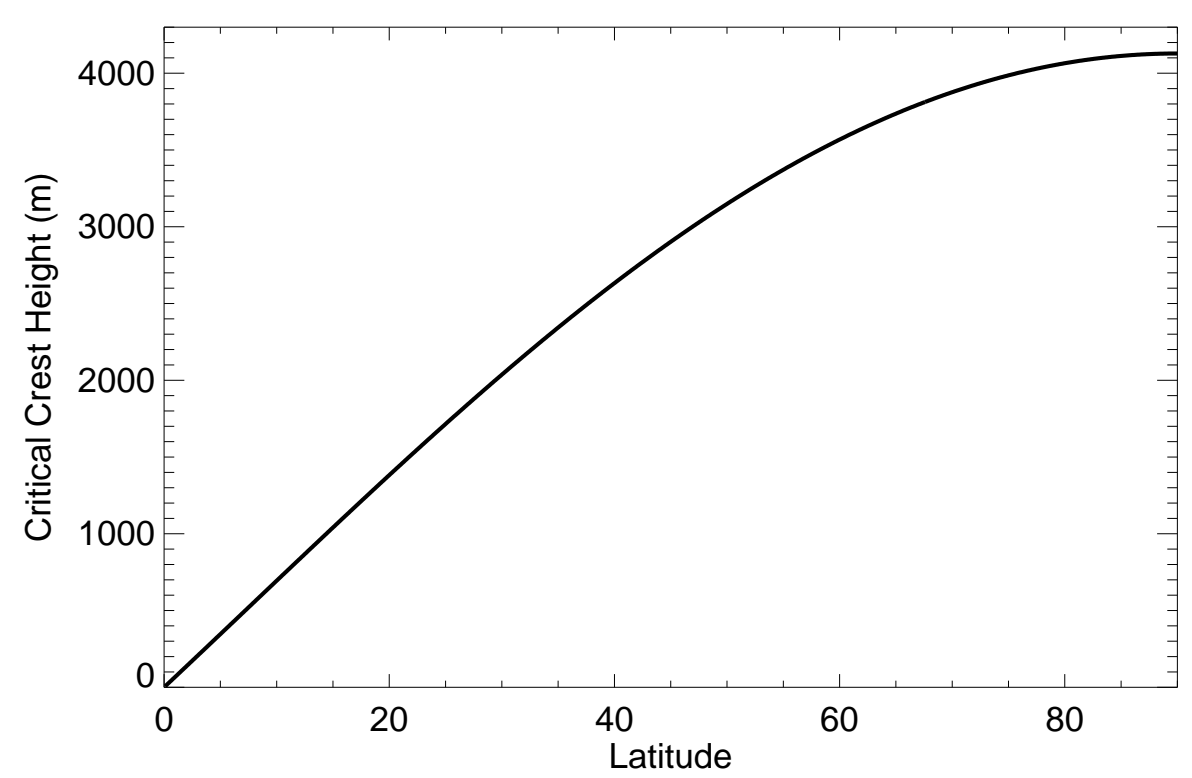

Figure 3.4: Critical crest height as a function of latitude for reference state 1.

and $\theta=\theta_{B}$. Using a Gaussian shaped obstacle we can write

$$
\left(\frac{g}{N_{1} \theta_{B}}\right)^{2}=\frac{g H_{c} a}{\sqrt{2}} \int_{0}^{\infty} \kappa^{2} e^{-(a k / 2)^{2}}\left(\frac{1+e^{-2 \kappa\left(\theta_{T}-\theta_{B}\right)}}{1+\kappa \theta_{B}+\left(1-\kappa \theta_{B}\right) e^{-2 \kappa\left(\theta_{T}-\theta_{B}\right)}}\right) d k,
$$

or, changing the integration variable

$$
\frac{1}{H_{c}}=\frac{a f \theta_{B} N_{1}}{\sqrt{2}}\left(\frac{\theta_{B} N_{1}}{g}\right)^{2} \int_{0}^{\infty} \kappa^{2} \frac{e^{-\left(a f \theta_{B} N_{1} \kappa / 2 g\right)^{2}}\left(1+e^{-2 \kappa\left(\theta_{T}-\theta_{B}\right)}\right)}{1+\kappa \theta_{B}+\left(1-\kappa \theta_{B}\right) e^{-2 \kappa\left(\theta_{T}-\theta_{B}\right)}} d \kappa .
$$

Theoretically this predicts the maximum height an obstacle in the atmosphere can have before it is required to puncture one or more isentropic surfaces. The significant dependence on latitude apparent in Fig. 3.4 becomes important for mountain ranges that have a large north-south extent such as the Rocky Mountains and the Andes. Mountains at low latitudes have a much smaller critical crest height and therefore can be expected to penetrate through the lower isentropes even for relatively weak heating cases. The main concentration of the highest peaks in the Rocky Mountains occurs in the mid-latitudes while much of the Andes are closer to the equator. The critical crest height also depends on the width of the mountain, as shown in Fig. 3.5. The Gaussian half-width of the idealized mountain is plotted on the horizontal axis for five different latitudes. Wide ridges or mountains have a higher critical crest height than do narrow mountains at a given latitude. Both of 


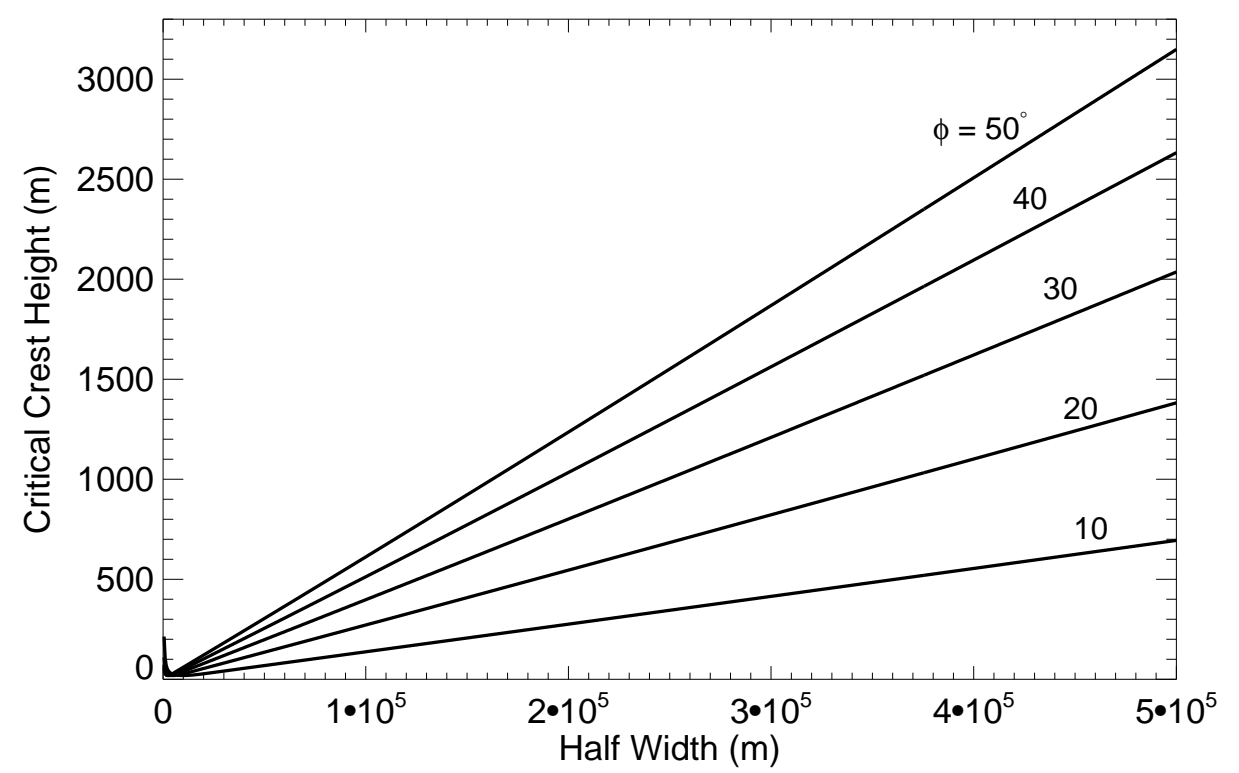

Figure 3.5: Critical crest height as a function of Gaussian halfwidth for reference state 1 at five different latitudes.

these figures indicate that the Andes will tend to have a significantly lower critical crest height than the Rocky Mountains.

Note that this has been computed for an atmosphere at rest with no heating. This parameter $H_{c}$ then cannot be directly compared with the environment we observe, but it should give us a reasonable feel for which topographic regions the lower bound must break through isentropes. 


\section{Chapter 4}

\section{TOPOGRAPHICALLY BOUND BALANCED MOTIONS ON THE SPHERE}

\subsection{Introduction}

In general there are several methods of solution for the elliptic system that was derived in the previous chapter. As shown there, one approach is to use Fourier tran sforms in the horizontal direction and then use Green's functions to solve the vertical structure equation. This method worked well and the solutions (3.41-3.42) were used to generate the Fig. 3.3. However, it was unclear how to generalize this method to a spherical domain. Here we present a different derivation that allows for solutions to be found on the sphere. This method starts with a transform in the vertical dimension. This leads to a horizontal structure equation that can be solved using spheroidal harmonics. Instead of the solution being composed of Green's functions as in the last chapter, the solution here is composed of vertical structure functions that satisfy a Sturm-Liouville problem. For clarity and comparison with the previous chapter, the derivation will be done first on an $f$-plane, after which the generalization to the sphere will be straightforward.

\subsection{Invertibility principle}

To begin, the governing equation of the flow (3.11) is rewritten in terms of the Montgomery potential anomaly $M^{\prime}(x, \theta)$. Equation (3.11) then takes the form

$$
\frac{\partial^{2} M^{\prime}}{\partial x^{2}}+\left(\frac{f^{2} \theta_{B}^{2} N_{B}^{2} P}{g^{2} \tilde{P}}\right) \frac{\partial^{2} M^{\prime}}{\partial \theta^{2}}=f^{2}\left(\frac{P}{\tilde{P}}-1\right) .
$$


As we did for equation (3.11) we assume that, except in the massless layer, the potential vorticity is uniform on each isentropic surface, i.e., $P(x, \theta)=\tilde{P}(\theta)$ for $\theta_{S}(x)<\theta \leq \theta_{T}$, so that (4.1) simplifies to the top entry in (4.2) below. In the massless layer, the pseudodensity vanishes, i.e., $\partial p / \partial \theta=0$, or equivalently $\partial \Pi / \partial \theta=0$, which can be written as the second line in (4.2). Alternatively (4.1) could be multiplied by $\tilde{P} / P$ and then the limit taken as $P \rightarrow \infty$. As for boundary conditions, we require that $M^{\prime}$ approaches zero in the far-field, which is expressed in (4.3). We also require that the upper boundary is both an isentropic $\left(\theta=\theta_{T}\right)$ and isobaric surface, which is expressed in (4.4). To formulate the lower boundary condition we subtract $\tilde{M}-\theta(\partial \tilde{M} / \partial \theta)=\tilde{\phi}$ from $M-\theta(\partial M / \partial \theta)=\phi$ to obtain $M^{\prime}-\theta\left(\partial M^{\prime} / \partial \theta\right)=\phi-\tilde{\phi}$, which, when applied at $\theta=\theta_{B}$ yields (4.5). In summary, the elliptic problem is

$$
\begin{array}{r}
\frac{\partial^{2} M^{\prime}}{\partial x^{2}}+\left(\frac{f \theta_{B} N_{B}}{g}\right)^{2} \frac{\partial^{2} M^{\prime}}{\partial \theta^{2}}=0 \text { for } \theta_{S}(x)<\theta \leq \theta_{T}, \\
\left(\frac{f \theta_{B} N_{B}}{g}\right)^{2} \frac{\partial^{2} M^{\prime}}{\partial \theta^{2}}=f^{2} \text { for } \theta_{B} \leq \theta<\theta_{S}(x),
\end{array}
$$

with boundary conditions

$$
\begin{gathered}
M^{\prime} \rightarrow 0 \text { as } x \rightarrow \infty, \\
\frac{\partial M^{\prime}}{\partial \theta}=0 \text { at } \theta=\theta_{T}, \\
M^{\prime}-\theta \frac{\partial M^{\prime}}{\partial \theta}=\Phi(x) \text { at } \theta=\theta_{B} .
\end{gathered}
$$

Two interesting special cases occur: (i) $\theta_{S}(x)=\theta_{B}$ and $\Phi(x) \neq 0$; (ii) $\theta_{S}(x) \neq \theta_{B}$ and $\Phi(x)=$ 0. In special case (i), all the orographic features are isentropic, so only the top line in (4.2) is relevant and the entire forcing comes from the specified $\Phi(x)$ field. In special case (ii), there are no orographic features but potential temperature varies along the flat lower boundary, so that the entire forcing comes from the massless layers associated with the specified $\theta_{S}(x)$ field. Special case (ii) is useful in understanding the flow patterns forced by land-sea contrast on an Earth-like planet with no mountains. 


\subsection{Solution of the invertibility principle on the $f$-plane via transform methods}

To solve the invertibility problem (4.2)-(4.5) we first introduce the vertical transform pair

$$
\begin{gathered}
M_{\ell}^{\prime}(x)=\frac{1}{\theta_{T}-\theta_{B}} \int_{\theta_{B}}^{\theta_{T}} M^{\prime}(x, \theta) V_{\ell}(\theta) d \theta, \\
M^{\prime}(x, \theta)=\sum_{\ell=0}^{\infty} M_{\ell}^{\prime}(x) V_{\ell}(\theta),
\end{gathered}
$$

where $V_{\ell}(\theta)$ are the vertical structure functions, which satisfy the Sturm-Liouville problem

$$
\begin{gathered}
\frac{d^{2} V_{\ell}}{d \theta^{2}}+\left(\frac{g}{\theta_{B} N_{B} c_{\ell}}\right)^{2} V_{\ell}=0, \\
\frac{d V_{\ell}}{d \theta}=0 \quad \text { at } \quad \theta=\theta_{T}, \\
V_{\ell}-\theta \frac{d V_{\ell}}{d \theta}=0 \quad \text { at } \quad \theta=\theta_{B},
\end{gathered}
$$

with the integer $\ell$ denoting the index of the vertical mode. Only solutions with $c_{\ell}^{2}>0$ are possible. For $c_{\ell}^{2}>0$ the solution of (4.8) satisfying the upper boundary condition (4.9) is

$$
V_{\ell}(\theta)=A_{\ell} \cos \left(\frac{g\left(\theta_{T}-\theta\right)}{\theta_{B} N_{B} c_{\ell}}\right)
$$

where

$$
A_{\ell}=\sqrt{2}\left\{1+\frac{\sin \left[2 g\left(\theta_{T}-\theta_{B}\right) /\left(\theta_{B} N_{B} c_{\ell}\right)\right]}{2 g\left(\theta_{T}-\theta_{B}\right) /\left(\theta_{B} N_{B} c_{\ell}\right)}\right\}^{-1 / 2}
$$

is the normalization factor. The lower boundary condition (4.10) is satisfied if $c_{\ell}$ satisfies the transcendental equation

$$
\tan \left(\frac{g\left(\theta_{T}-\theta_{B}\right)}{\theta_{B} N_{B} c_{\ell}}\right)-\frac{N_{B} c_{\ell}}{g}=0 .
$$

Approximate solutions of (4.13) are given by

$$
c_{\ell} \approx \frac{g\left(\theta_{T}-\theta_{B}\right)}{\ell \pi \theta_{B} N_{B}}
$$

The first eleven solutions of (4.13), indexed $\ell=0,1,2, \cdots, 10$, are given in the second column of Table 4.1, while the approximate solutions are given in the third column. The vertical structure functions $V_{\ell}(\theta)$ satisfy the orthonormality relation

$$
\frac{1}{\theta_{T}-\theta_{B}} \int_{\theta_{B}}^{\theta_{T}} V_{\ell}(\theta) V_{\ell^{\prime}}(\theta) d \theta= \begin{cases}1 & \ell^{\prime}=\ell \\ 0 & \ell^{\prime} \neq \ell\end{cases}
$$


Note that (4.6) can be obtained by multiplying (4.7) by $V_{\ell^{\prime}}(\theta)$, integrating over $\theta$, and then using the orthonormality relation (4.15).

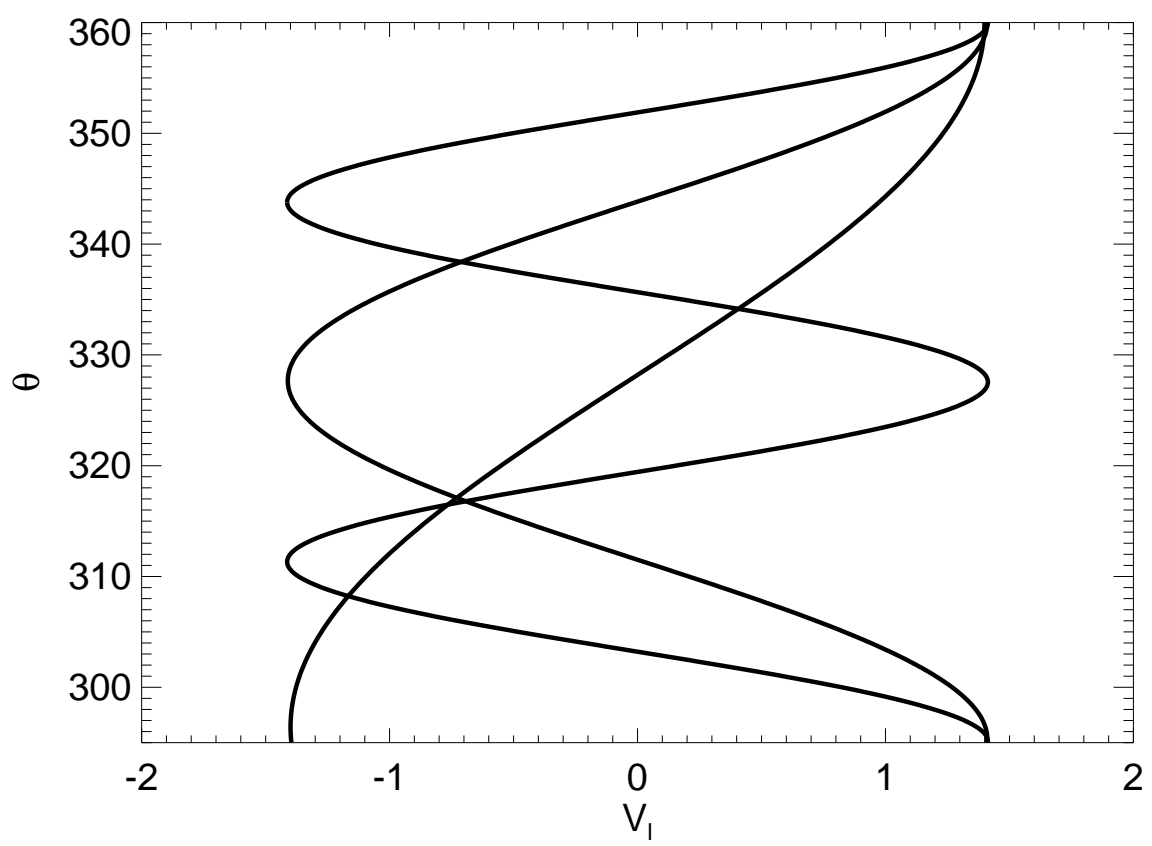

Figure 4.1: Vertical structure functions $V_{\ell}(\theta)$ for $\ell=0,1,2,3$.

To take the vertical transform of (4.2) we first multiply the bottom line in (4.2) by $V_{\ell}(\theta)$ and integrate from $\theta_{B}$ to $\theta_{S}(x)$, thereby obtaining

$$
\int_{\theta_{B}}^{\theta_{S}(x)}\left(\frac{f \theta_{B} N_{B}}{g}\right)^{2} \frac{\partial^{2} M^{\prime}}{\partial \theta^{2}} V_{\ell} d \theta=\int_{\theta_{B}}^{\theta_{S}(x)} f^{2} V_{\ell} d \theta .
$$

Similarly, multiplying the top line in (4.2) by $V_{\ell}(\theta)$ and integrating from $\theta_{S}(x)$ to $\theta_{T}$, we obtain

$$
\int_{\theta_{B}}^{\theta_{T}} \frac{\partial^{2} M^{\prime}}{\partial x^{2}} V_{\ell} d \theta+\int_{\theta_{S}(x)}^{\theta_{T}}\left(\frac{f \theta_{B} N_{B}}{g}\right)^{2} \frac{\partial^{2} M^{\prime}}{\partial \theta^{2}} V_{\ell} d \theta=\int_{\theta_{B}}^{\theta_{S}(x)} \frac{\partial^{2} M^{\prime}}{\partial x^{2}} V_{\ell} d \theta
$$

Taking the sum of (4.16) and (4.17), we obtain

$$
\int_{\theta_{B}}^{\theta_{T}}\left\{\frac{\partial^{2} M^{\prime}}{\partial x^{2}}+\left(\frac{f \theta_{B} N_{B}}{g}\right)^{2} \frac{\partial^{2} M^{\prime}}{\partial \theta^{2}}\right\} V_{\ell} d \theta=\int_{\theta_{B}}^{\theta_{S}(x)}\left(f^{2}+\frac{\partial^{2} M^{\prime}}{\partial x^{2}}\right) V_{\ell} d \theta .
$$

Integrating the $\left(\partial^{2} M^{\prime} / \partial \theta^{2}\right) V_{\ell}$ term by parts twice, using the boundary conditions (4.4), (4.5), (4.9), and (4.10), and finally recalling the transform relation (4.6), we obtain

$$
\frac{d^{2} M_{\ell}^{\prime}}{d x^{2}}-\frac{f^{2}}{c_{\ell}^{2}} M_{\ell}^{\prime}=F_{\ell}
$$


where

$$
F_{\ell}(x)=\frac{1}{\theta_{T}-\theta_{B}} \int_{\theta_{B}}^{\theta_{S}(x)}\left(f^{2}+\frac{\partial^{2} M^{\prime}}{\partial x^{2}}\right) V_{\ell} d \theta-\left(\frac{f N_{B}}{g}\right)^{2} \frac{\theta_{B} V_{\ell}\left(\theta_{B}\right)}{\theta_{T}-\theta_{B}} \Phi(x) .
$$

To solve the horizontal structure problem (4.19) we now introduce the Fourier transform pair

$$
\begin{aligned}
\hat{M}_{\ell}^{\prime}(k) & =\frac{2}{\pi} \int_{0}^{\infty} M_{\ell}^{\prime}(x) \cos (k x) d x, \\
M_{\ell}^{\prime}(x) & =\int_{0}^{\infty} \hat{M}_{\ell}^{\prime}(k) \cos (k x) d k,
\end{aligned}
$$

where $k$ is the horizontal wavenumber. A similar cosine transform pair exists for the surface geopotential $\Phi(x)$ and its transform $\hat{\Phi}(k)$. Taking the Fourier cosine transform of (4.19) we obtain

$$
\hat{M}_{\ell}^{\prime}(k)=-\frac{c_{\ell}^{2} \hat{F}_{\ell}(k)}{f^{2}+c_{\ell}^{2} k^{2}}
$$

which is the spectral space solution of the original invertibility problem (4.2)-(4.5). This solution can be transformed back to physical space through the use of (4.7) and (4.22), which results in

$$
M^{\prime}(x, \theta)=-\sum_{\ell=0}^{\infty}\left\{\int_{0}^{\infty} \frac{c_{\ell}^{2} \hat{F}_{\ell}(k)}{f^{2}+c_{\ell}^{2} k^{2}} \cos (k x) d k\right\} V_{\ell}(\theta) .
$$

In the next section we examine this solution for the special case of an isentropic mountain.

\subsection{The case of an isentropic mountain}

Now consider the special case in which the mountain is an isentrope, i.e., $\theta_{S}(x)=\theta_{B}$ so that (4.20) reduces to

$$
F_{\ell}(x)=-\left(\frac{f N_{B}}{g}\right)^{2} \frac{\theta_{B} V_{\ell}\left(\theta_{B}\right)}{\theta_{T}-\theta_{B}} \Phi(x)
$$

For all the calculations presented in this section we have chosen the geopotential on the lower boundary as

$$
\Phi(x)=g H e^{-x^{2} / a^{2}}
$$

where the constants $H$ and $a$ respectively specify the mountain height and width. The Fourier cosine transform of (4.26) yields

$$
\hat{\Phi}(k)=\frac{g H a}{\sqrt{2}} e^{-a^{2} k^{2} / 4},
$$


so that

$$
\hat{F}_{\ell}(k)=-\left(\frac{f N_{B}}{g}\right)^{2} \frac{\theta_{B} V_{\ell}\left(\theta_{B}\right)}{\theta_{T}-\theta_{B}} \frac{g H a}{\sqrt{2}} e^{-a^{2} k^{2} / 4} .
$$

Plugging this into (4.23) gives

$$
\hat{M}_{\ell}^{\prime}(k)=\left(\frac{c_{\ell}^{2}}{f^{2}+c_{\ell}^{2} k^{2}}\right)\left(\frac{f N_{B}}{g}\right)^{2} \frac{\theta_{B} V_{\ell}\left(\theta_{B}\right)}{\theta_{T}-\theta_{B}} \hat{\Phi}(k) .
$$

The Montgomery potential in $(x, \theta)$ space is then obtained using (4.7)

\subsection{Invertibility principle on the sphere}

In this section the previous argument is generalized to a spherical domain. Using the longitude $\lambda$ and the latitude $\varphi$ as horizontal coordinates and the potential temperature $\theta$ as the vertical coordinate, and denoting the zonal component of the flow by $u$ and the meridional component by $v$, the governing equations for inviscid flow on the sphere take the form

$$
\begin{gathered}
\frac{D u}{D t}-\left(2 \Omega \sin \varphi+\frac{u \tan \varphi}{a}\right) v+\frac{\partial M}{a \cos \varphi \partial \lambda}=0, \\
\frac{D v}{D t}+\left(2 \Omega \sin \varphi+\frac{u \tan \varphi}{a}\right) u+\frac{\partial M}{a \partial \varphi}=0, \\
\Pi=\frac{\partial M}{\partial \theta}, \\
\frac{D \sigma}{D t}+\sigma\left(\frac{\partial u}{a \cos \varphi \partial \lambda}+\frac{\partial(v \cos \varphi)}{a \cos \varphi \partial \varphi}+\frac{\partial \dot{\theta}}{\partial \theta}\right)=0,
\end{gathered}
$$

where $\Omega$ and $a$ are the Earth's rotation rate and radius, $M=\theta \Pi+\phi$ is the Montgomery potential, $\phi$ is the geopotential, $\Pi(p)=c_{p}\left(p / p_{0}\right)^{\kappa}$ is the Exner function, $\sigma=-(1 / g)(\partial p / \partial \theta)$ is the pseudo-

density, and $(D / D t)=(\partial / \partial t)+u(\partial / a \cos \varphi \partial \lambda)+v(\partial / a \partial \varphi)+\dot{\theta}(\partial / \partial \theta)$ is the material derivative. The variables $u, v, M, p$ are functions of $(\lambda, \varphi, \theta, t)$.

The potential vorticity principle, derived from (4.30), (4.31) and (4.33), is

$$
\sigma \frac{D P}{D t}=-\frac{\partial v}{\partial \theta} \frac{\partial \dot{\theta}}{a \cos \phi \partial \lambda}+\frac{\partial u}{\partial \theta} \frac{\partial \dot{\theta}}{a \partial \varphi}+\left(2 \Omega \mu+\nabla^{2} \psi\right) \frac{\partial \dot{\theta}}{\partial \theta}
$$

where the potential vorticity is defined by

$$
P=\left(2 \Omega \mu+\nabla^{2} \psi\right)\left(-\frac{1}{g} \frac{\partial p}{\partial \theta}\right)^{-1},
$$


with $\mu=\sin \varphi$ and with the relative vorticity expressed as the horizontal Laplacian of the streamfunction, i.e.,

$$
\nabla^{2} \psi=\frac{\partial^{2} \psi}{a^{2}\left(1-\mu^{2}\right) \partial \lambda^{2}}+\frac{\partial}{a^{2} \partial \mu}\left(\left(1-\mu^{2}\right) \frac{\partial \psi}{\partial \mu}\right)
$$

As in the derivation on the $f$-plane, in the reference state the flow is assumed to vanish. The ratio of the potential vorticity (4.35) to the reference state potential vorticity is given by

$$
2 \Omega \mu \frac{P}{\tilde{P}}=\left(2 \Omega \mu+\nabla^{2} \psi\right)\left(\frac{\partial \tilde{\Pi} / \partial \theta}{\partial \Pi / \partial \theta}\right) .
$$

To convert (4.37) into an invertibility principle, we need to formulate a balance condition between the nondivergent wind field $\psi$ and the anomaly of the Montgomery potential, defined by $M^{\prime}=M-\tilde{M}$, where $\tilde{M}(\theta)$ is hydrostatically related to $\tilde{\Pi}(\theta)$ via $d \tilde{M} / d \theta=\tilde{\Pi}$. The balance condition used here is an approximation of the linear balance condition $\nabla \cdot(2 \Omega \mu \nabla \psi)=\nabla^{2} M^{\prime}$. The approximation is obtained by considering $2 \Omega \mu$ to be slowly varying, so that the linear balance condition simplifies to $\nabla^{2}\left(M^{\prime}-2 \Omega \mu \psi\right)=0$, from which the local linear balance condition $M^{\prime}=$ $2 \Omega \mu \psi$ then follows. Discussions of the accuracy of this local linear balance condition can be found in Schubert and Masarik (2006), Verkley (2009), and Schubert et al. (2009). Since $\Pi-\tilde{\Pi}=\Pi^{\prime}=$ $\partial M^{\prime} / \partial \theta=2 \Omega \mu(\partial \psi / \partial \theta),(4.37)$ can now be written in the form

$$
\nabla^{2} \psi+\left(\frac{\theta N 2 \Omega \mu}{g}\right)^{2} \frac{P}{\tilde{P}} \frac{\partial^{2} \psi}{\partial \theta^{2}}=2 \Omega \mu\left(\frac{P}{\tilde{P}}-1\right)
$$

where we have defined the reference state buoyancy frequency $N(\theta)$ by

$$
N^{2}(\theta)=\frac{g^{2}}{\theta^{2}}\left(-\frac{d \tilde{\Pi}}{d \theta}\right)^{-1}
$$

For the reference state we assume that the buoyancy frequency $N(\theta)$ is inversely proportional to $\theta$, i.e., $N(\theta)=N_{B} \theta_{B} / \theta$, where $N_{B}$ and $\theta_{B}$ are constants.

We shall require that (4.38) holds on a sphere that includes topographic features whose geopotential is specified by $\Phi(\lambda, \mu)$. We assume that, due to radiative processes, the potential temperature varies along these topographic features according to the specified function $\theta_{S}(\lambda, \mu)$. As in chapter 3 the region $\theta_{B}<\theta<\theta_{S}(\lambda, \mu)$ is the massless layer. 
We now assume that, except in the massless layer, the potential vorticity is equal to the reference value, i.e., $P(\lambda, \mu, \theta)=\tilde{P}(\mu, \theta)$, so that (4.38) simplifies to the top entry in (4.40) below. In the massless layer, the pseudodensity vanishes, i.e., $\partial p / \partial \theta=0$, or equivalently, $\partial \Pi / \partial \theta=0$, which can be written as the bottom entry in (4.40). As for boundary conditions, we require that the upper boundary is both an isentropic $\left(\theta=\theta_{T}\right)$ and isobaric surface, i.e., $\Pi=\tilde{\Pi}$, which, using the hydrostatic equation and the balance condition, can be written as $\partial M^{\prime} / \partial \theta=2 \Omega \mu(\partial \psi / \partial \theta)=$ 0 , or simply as (4.41) below. At the bottom boundary the geopotential is $\phi=\Phi(\lambda, \mu)$, so that $\theta[\partial(M-\tilde{M}) / \partial \theta]=\theta(\Pi-\tilde{\Pi})=M-\tilde{M}-\Phi$, or equivalently, $2 \Omega \mu[\psi-\theta(\partial \psi / \partial \theta)]=\Phi$. In summary, the elliptic problem for $\psi$ is

$$
\begin{array}{r}
\nabla^{2} \psi+\left(\frac{2 \Omega \mu \theta_{B} N_{B}}{g}\right)^{2} \frac{\partial^{2} \psi}{\partial \theta^{2}}=0 \text { for } \theta_{S}(\lambda, \mu)<\theta \leq \theta_{T} \\
\left(\frac{2 \Omega \mu \theta_{B} N_{B}}{g}\right)^{2} \frac{\partial^{2} \psi}{\partial \theta^{2}}=2 \Omega \mu \text { for } \theta_{B} \leq \theta<\theta_{S}(\lambda, \mu),
\end{array}
$$

with boundary conditions

$$
\begin{gathered}
\frac{\partial \psi}{\partial \theta}=0 \text { at } \theta=\theta_{T}, \\
\psi-\theta \frac{\partial \psi}{\partial \theta}=\Psi \text { at } \theta=\theta_{B},
\end{gathered}
$$

where $\Psi$ is defined in terms of $\Phi$ by $\Phi=2 \Omega \mu \Psi$.

\subsection{Solution of the invertibility problem via transform methods on the sphere}

To solve the invertibility problem (4.40)-(4.42) we introduce the vertical transform pair

$$
\begin{gathered}
\psi_{\ell}(\lambda, \mu)=\frac{1}{\theta_{T}-\theta_{B}} \int_{\theta_{B}}^{\theta_{T}} \psi(\lambda, \mu, \theta) V_{\ell}(\theta) d \theta \\
\psi(\lambda, \mu, \theta)=\sum_{\ell=0}^{\infty} \psi_{\ell}(\lambda, \mu) V_{\ell}(\theta),
\end{gathered}
$$

where $V_{\ell}(\theta)$ are the vertical structure functions introduced earlier.

To take the vertical transform of (4.40) we first multiply the bottom line in (4.40) by $V_{\ell}(\theta)$ and integrate from $\theta_{B}$ to $\theta_{S}(\lambda, \mu)$, thereby obtaining

$$
\int_{\theta_{B}}^{\theta_{S}(\lambda, \mu)}\left(\frac{2 \Omega \mu \theta_{B} N_{B}}{g}\right)^{2} \frac{\partial^{2} \psi}{\partial \theta^{2}} V_{\ell} d \theta=\int_{\theta_{B}}^{\theta_{S}(\lambda, \mu)} 2 \Omega \mu V_{\ell} d \theta
$$


Similarly, multiplying the top line in (4.40) by $V_{\ell}(\theta)$ and integrating from $\theta_{S}(\lambda, \mu)$ to $\theta_{T}$, we obtain

$$
\int_{\theta_{B}}^{\theta_{T}} \nabla^{2} \psi V_{\ell} d \theta+\int_{\theta_{S}(\lambda, \mu)}^{\theta_{T}}\left(\frac{2 \Omega \mu \theta_{B} N_{B}}{g}\right)^{2} \frac{\partial^{2} \psi}{\partial \theta^{2}} V_{\ell} d \theta=\int_{\theta_{B}}^{\theta_{S}(\lambda, \mu)} \nabla^{2} \psi V_{\ell} d \theta
$$

Taking the sum of (4.45) and (4.46), we obtain

$$
\int_{\theta_{B}}^{\theta_{T}}\left\{\nabla^{2} \psi+\left(\frac{2 \Omega \mu \theta_{B} N_{B}}{g}\right)^{2} \frac{\partial^{2} \psi}{\partial \theta^{2}}\right\} V_{\ell} d \theta=\int_{\theta_{B}}^{\theta_{S}(\lambda, \mu)}\left(2 \Omega \mu+\nabla^{2} \psi\right) V_{\ell} d \theta .
$$

Integrating the $\left(\partial^{2} \psi / \partial \theta^{2}\right) V_{\ell}$ term by parts twice, using the boundary conditions (4.9), (4.10), (4.41), and (4.42), and finally recalling the transform relation (4.43), we obtain

$$
\nabla^{2} \psi_{\ell}-\frac{\epsilon_{\ell} \mu^{2}}{a^{2}} \psi_{\ell}=F_{\ell}
$$

where

$$
\epsilon_{\ell}=\left(\frac{2 \Omega a}{c_{\ell}}\right)^{2}
$$

is Lamb's parameter and

$$
\begin{aligned}
F_{\ell}(\lambda, \mu) & =\frac{1}{\theta_{T}-\theta_{B}} \int_{\theta_{B}}^{\theta_{S}(\lambda, \mu)}\left(2 \Omega \mu+\nabla^{2} \psi\right) V_{\ell}(\theta) d \theta \\
& -\left(\frac{2 \Omega \mu \theta_{B} N_{B}}{g}\right)^{2} \frac{V_{\ell}\left(\theta_{B}\right)}{\left(\theta_{T}-\theta_{B}\right) \theta_{B}} \Psi(\lambda, \mu)
\end{aligned}
$$

The first term on the right hand side can be interpreted as a measure of the "absolute isentropic vorticity in the massless layer."

To solve the horizontal structure problem (4.48) we now introduce the spheroidal harmonic transform pair

$$
\begin{gathered}
\psi_{\ell m n}=\frac{1}{4 \pi} \int_{-1}^{1} \int_{0}^{2 \pi} \psi_{\ell}(\lambda, \mu) S_{m n}^{*}\left(\epsilon_{\ell} ; \lambda, \mu\right) d \lambda d \mu, \\
\psi_{\ell}(\lambda, \mu)=\sum_{m=-\infty}^{\infty} \sum_{n=|m|}^{\infty} \psi_{\ell m n} S_{m n}\left(\epsilon_{\ell} ; \lambda, \mu\right),
\end{gathered}
$$

where $S_{m n}\left(\epsilon_{\ell} ; \lambda, \mu\right)$ are the spheroidal harmonic functions, which satisfy

$$
\nabla^{2} S_{m n}-\frac{\epsilon_{\ell} \mu^{2}}{a^{2}} S_{m n}=-\frac{\alpha_{m n}\left(\epsilon_{\ell}\right)}{a^{2}} S_{m n}
$$

where the integer $m$ is the zonal wavenumber, the integer $n$ is the total wavenumber, and the values $\alpha_{m n}\left(\epsilon_{\ell}\right)$ are the eigenvalues of the spheroidal harmonic operator. The orthonormality relation for 
spheroidal harmonics is

$$
\frac{1}{4 \pi} \int_{-1}^{1} \int_{0}^{2 \pi} S_{m n}\left(\epsilon_{\ell} ; \lambda, \mu\right) S_{m^{\prime} n^{\prime}}^{*}\left(\epsilon_{\ell} ; \lambda, \mu\right) d \lambda d \mu= \begin{cases}1 & \left(m^{\prime}, n^{\prime}\right)=(m, n) \\ 0 & \left(m^{\prime}, n^{\prime}\right) \neq(m, n) .\end{cases}
$$

Note that (4.51) can be obtained by multiplying (4.52) by $S_{m^{\prime} n^{\prime}}^{*}\left(\epsilon_{\ell} ; \lambda, \mu\right)$, integrating over $\lambda$ and $\mu$, and then using the orthonormality relation (4.54). A concise summary of spheroidal harmonics is given in Abramowitz and Stegun (1965, pages 751-769). A more extensive discussion is given in Flammer (1957). The Mathematica software package (versions 6 and higher) has built-in support for computing spheroidal harmonic eigenvalues and eigenfunctions.

To take the spheroidal harmonic transform of (4.48) we multiply it by $S_{m n}^{*}\left(\epsilon_{\ell} ; \lambda, \mu\right)$ and integrate over the entire sphere, thereby obtaining

$$
\int_{-1}^{1} \int_{0}^{2 \pi} \psi_{\ell}\left(\nabla^{2} S_{m n}^{*}-\frac{\epsilon_{\ell} \mu^{2}}{a^{2}} S_{m n}^{*}\right) d \lambda d \mu=\int_{-1}^{1} \int_{0}^{2 \pi} F_{\ell} S_{m n}^{*} d \lambda d \mu
$$

where we have used $\iint S_{m n}^{*} \nabla^{2} \psi_{\ell} d \lambda d \mu=\iint \psi_{\ell} \nabla^{2} S_{m n}^{*} d \lambda d \mu$. Using the complex conjugate of (4.53), the transform relation (4.51), and noting that $F_{\ell}(\lambda, \mu)$ and $F_{\ell m n}$ are related by a transform pair identical to (4.51) and (4.52), we can simplify (4.55) to

$$
\psi_{\ell m n}=\frac{F_{\ell m n}}{\alpha_{m n}\left(\epsilon_{\ell}\right)}
$$

which is the spectral space solution of the original invertibility problem (4.40)-(4.42). This solution can be transformed back to physical space through the use of (4.44) and (4.52), which results in

$$
\psi(\lambda, \mu, \theta)=\sum_{\ell=0}^{\infty} \sum_{m=-\infty}^{\infty} \sum_{n=|m|}^{\infty} \frac{F_{\ell m n}}{\alpha_{m n}\left(\epsilon_{\ell}\right)} V_{\ell}(\theta) S_{m n}\left(\epsilon_{\ell} ; \lambda, \mu\right) .
$$

Once $\psi(\lambda, \mu, \theta)$ is known, the wind field can easily be computed because we have assumed the wind field is nondivergent.

\subsection{Discussion of various solution methods}

Why has the same physical system been written as two distinct elliptic problems as given by (3.12-3.14) and (4.2-4.5)? Recall that the elliptic problem from chapter 2 is solved with Fourier 
transforms. This approach led to a solution but the transforms were complicated by the boundary between the atmosphere and the massless layer and it was not clear how to generalize the results to a spherical domain. However, rewriting the system in terms of the perturbation Montgomery potential as in (4.2-4.5) leads to an elegant transform process and the spherical generalization can be derived. This is why two distinct elliptic problems were used.

The next chapter solves the invertibility principle using finite-difference approximations. In this case the elliptic problem is written in terms of the full Montgomery potential $M$. Often when using transforms, variables that represent perturbations (like $\Pi^{\prime}$ and $M^{\prime}$ ) which approach zero in the far field make the definition of boundary conditions convenient. With a finite-difference system this is not necessary because there is more flexibility in the choice of boundary conditions. In the following chapter there is no need to use perturbation variables and so the full Montgomery potential is used. Thus three different elliptic systems have been used to represent the same physical system. It is straightforward to show that they are all equivalent. 


\begin{tabular}{|c|c|c|}
\hline \hline$\ell$ & $\begin{array}{c}c_{\ell}\left(\mathrm{m} \mathrm{s}^{-1}\right) \\
\text { Exact }\end{array}$ & $\begin{array}{c}c_{\ell}\left(\mathrm{m} \mathrm{s}^{-1}\right) \\
\text { Approximate }\end{array}$ \\
\hline \hline 0 & 365.2 & $\infty$ \\
1 & 51.52 & 52.64 \\
2 & 26.18 & 26.32 \\
3 & 17.50 & 17.55 \\
4 & 13.14 & 13.16 \\
5 & 10.52 & 10.53 \\
6 & 8.768 & 8.773 \\
7 & 7.517 & 7.520 \\
8 & 6.578 & 6.580 \\
9 & 5.847 & 5.849 \\
10 & 5.263 & 5.264 \\
\hline
\end{tabular}

Table 4.1: The spectrum of gravity wave speeds $c_{\ell}$, and approximate gravity wave speeds for the values of $\ell$ listed in the left column. The exact values of $c_{\ell}$ have been computed from (4.13) and the approximate values from (4.14) using $\theta_{B}=295 \mathrm{~K}, \theta_{T}=360 \mathrm{~K}, g=9.81 \mathrm{~m} \mathrm{~s}^{-2}$, and $\left(\theta_{B} / g\right) N_{B}^{2}=$ $5.1373 \mathrm{~K} \mathrm{~km}^{-1}$. 


\section{Chapter 5}

\section{NUMERICAL SOLUTIONS}

\subsection{Comparison between analytic and numerical solutions}

The analytically derived solutions given by (3.41) and (3.42) offer a 'clean' mathematical description of the influences orography and heating have on the balanced atmospheric wind and mass fields. These equations are valid for arbitrary orography and forcing by the function $F(k, \theta)$. However, to move from these solutions to a quantitative measure of how the wind and mass fields respond to a precise and specific forcing scenario, more work must be done. The process of computing wind fields for a given orography and a specified $F(k, \theta)$ has uncovered several mathematical subtleties that lead to further insight concerning the incorporation of a massless layer into a description of fluid systems. This chapter presents the results for the three simple cases discussed in chapter 1 and sketched in Fig. (1.1).

The original intent of using the transform method was to transform the system of equations (3.12)-(3.14) into spectral space where a solution would presumably be simpler to derive. However, the transformed system (3.22)-(3.24) contains the forcing term $F(k, \theta)$ which cannot be directly expressed in terms of specified quantities. When seeking to compute $v(x, \theta)$ and $\Pi^{\prime}(x, \theta)$ there are two problems with (3.22)-(3.24). First, these equations are partly in phy sical space and partly in spectral space. Second, it is clear that $v(x, \theta)$ cannot be computed from $\hat{v}(k, \theta)$ (using 3.16) because $\hat{v}(k, \theta)$ depends on $F(k, \theta)$, and $v(x, \theta)$ must be known to compute $F(x, \theta)$. What this implies is that we cannot simply dictate or choose a given $F(k, \theta)$ in the massless layer and then compute the resultant $v(x, \theta)$. Both $F(k, \theta)$ and $v(x, \theta)$ are unknowns. 
Further, because this is an elliptic system $F(k, \theta)$ depends not only on the geometry of the mountain, the potential temperature along the lower boundary, and $v(x, \theta)$ within the massless layer, but also on $v(x, \theta)$ throughout the domain. Specifying a given $F(k, \theta)$ at each grid point within the massless layer and then computing the resulting wind field will not work. However, $F(k, \theta)$ can be found through an iterative procedure. If we specify the lower heating, and the shape of the topography, a first approximation of $F(k, \theta)$ can be found. Then an approximate wind field is computed, and using the boundary conditions, topography, and wind field, the approximation of $F(k, \theta)$ can be recalculated and updated. This process is repeated until the wind field and $F(k, \theta)$ are no longer significantly changing with additional iterations.

To understand why our original solution method has broken down in this way, a closer look at (3.12)-(3.14) is helpful. It was originally assumed this was a linear, constant coefficient (when reference state 1 is assumed for $N(\theta)$ ) system and that accordingly, Fourier transforms would lead to a unique solution. This is not true. Equation (3.12) is a variable coefficient linear equation and as such, there is no guarantee that Fourier transforms will lead to a closed form solution. This can be seen if (3.12) is rewritten as

$$
\alpha \frac{\partial v}{\partial x}+\left(\frac{f \theta^{2} N^{2}}{g^{2}}\right) \frac{\partial \Pi^{\prime}}{\partial \theta}= \begin{cases}0 & x_{S}(\theta)<x<\infty \\ f & 0 \leq x<x_{S}(\theta)\end{cases}
$$

where $\alpha=1$ except in the massless layer where $\alpha=0$. The solutions derived using transforms led to insights concerning the behavior of the physical system, especially in the case of isentropic obstacles, but the transform did not produce easily computable solutions. It appears that the best way forward then is to use an iterative solution method. This is what follows.

\subsubsection{Finite difference discretization and successive over-relaxation}

When the system of equations is solved using finite difference approximations, transforms are not needed and it is unnecessary to separate the fields into a mean part and a perturbation part as was done in the previous two chapters. Here the system is written in terms of the full Montgomery potential. As before, potential vorticity is assumed to be conserved where the potential vorticity 
is defined by (3.8) with the notation the same as in previous chapters. Using the geostrophic and hydrostatic formulas $((\partial M / \partial x, \partial M / \partial \theta)=(f v, \Pi))$ the potential vorticity can be written as the following invertibility relation

$$
\begin{gathered}
\frac{g}{f \theta \rho P}\left(f^{2}+\frac{\partial^{2} M}{\partial x^{2}}\right)+\frac{\partial^{2} M}{\partial \theta^{2}}=0 \text { for } \theta_{S}(x)<\theta \leq \theta_{T} \\
\frac{\partial^{2} M}{\partial \theta^{2}}=0 \text { for } \theta_{B} \leq \theta<\theta_{S}(x)
\end{gathered}
$$

where the density $\rho$ is given in terms of $M$ by

$$
\rho=\frac{p_{0}}{R \theta}\left(\frac{1}{c_{p}} \frac{\partial M}{\partial \theta}\right)^{(1-\kappa) / \kappa} .
$$

Note that the full density is now being computed, which introduces a weak nonlinearity. The top line of (5.2) represents the system above the massless layer. In the massless layer, $P \rightarrow \infty$. In this limit the top line of (5.2) becomes the second line of (5.2) to govern $M$ in the massless layer. Along the left and right boundaries $M$ is required to equal the specified function $\tilde{M}(\theta)$. The upper boundary is required to be both an isentropic $\left(\theta=\theta_{T}\right)$ and an isobaric surface with a constant Exner function $\Pi_{T}$. To formulate the lower boundary condition we note that $M-\theta(\partial M / \partial \theta)=\phi$, and apply this at $\theta=\theta_{B}$. The boundary conditions are then expressed as

$$
\begin{gathered}
M=\tilde{M}(\theta) \text { at } x= \pm L, \\
\frac{\partial M}{\partial \theta}=\Pi_{T} \text { at } \theta=\theta_{T}, \\
M-\theta \frac{\partial M}{\partial \theta}=\phi_{S}(x) \text { at } \theta=\theta_{B} .
\end{gathered}
$$

As a first step towards an iterative solution of this elliptic problem, the numerical grid must be defined by discretizing the geometric domain. Our domain is divided into $J \times K$ grid cells with grid points given by $\left(x_{j}, \theta_{k}\right)=\left(-L+j \Delta x, \theta_{B}+k \Delta \theta\right)$ with $j=0,1, \ldots, J$ and $k=0,1, \ldots, K$, where $\Delta x=2 L / J$ and $\Delta \theta=\left(\theta_{T}-\theta_{B}\right) / K$. We then seek an approximate solution with gridpoint values $M_{j, k} \approx M\left(x_{j}, \theta_{k}\right)$ satisfying the discrete form of equation (5.2)

$$
\begin{gathered}
A_{j, k}\left[(f \Delta x)^{2}+M_{j-1, k}-2 M_{j, k}+M_{j+1, k}\right] \\
+M_{j, k-1}-2 M_{j, k}+M_{j, k+1}=0
\end{gathered}
$$


where the dimensionless coefficient $A_{j, k}$ is defined by

$$
A_{j, k}=\frac{g(\Delta \theta)^{2}}{f \theta_{k} \rho_{j, k} P_{j, k}(\Delta x)^{2}} .
$$

The discretized versions of (5.5) and (5.6) are

$$
\begin{gathered}
M_{j, K}-M_{j, K-1}=\Pi_{T} \Delta \theta \text { at } \theta=\theta_{T}, \\
M_{j, 0}-\frac{\theta_{B}}{\Delta \theta}\left(M_{j, 1}-M_{j, 0}\right)=\phi_{S}\left(x_{j}\right) \text { at } \theta=\theta_{B} .
\end{gathered}
$$

Note that the problem is nearly isotropic on the grid outside of the massless layer if $A_{j, k} \approx 1$, which can serve as a rough guide for the choice of the ratio $\Delta \theta / \Delta x$. Also note that (5.7) applies both outside and inside the massless layer, with $A_{j, k} \neq 0$ outside and $A_{j, k}=0$ inside the massless layer.

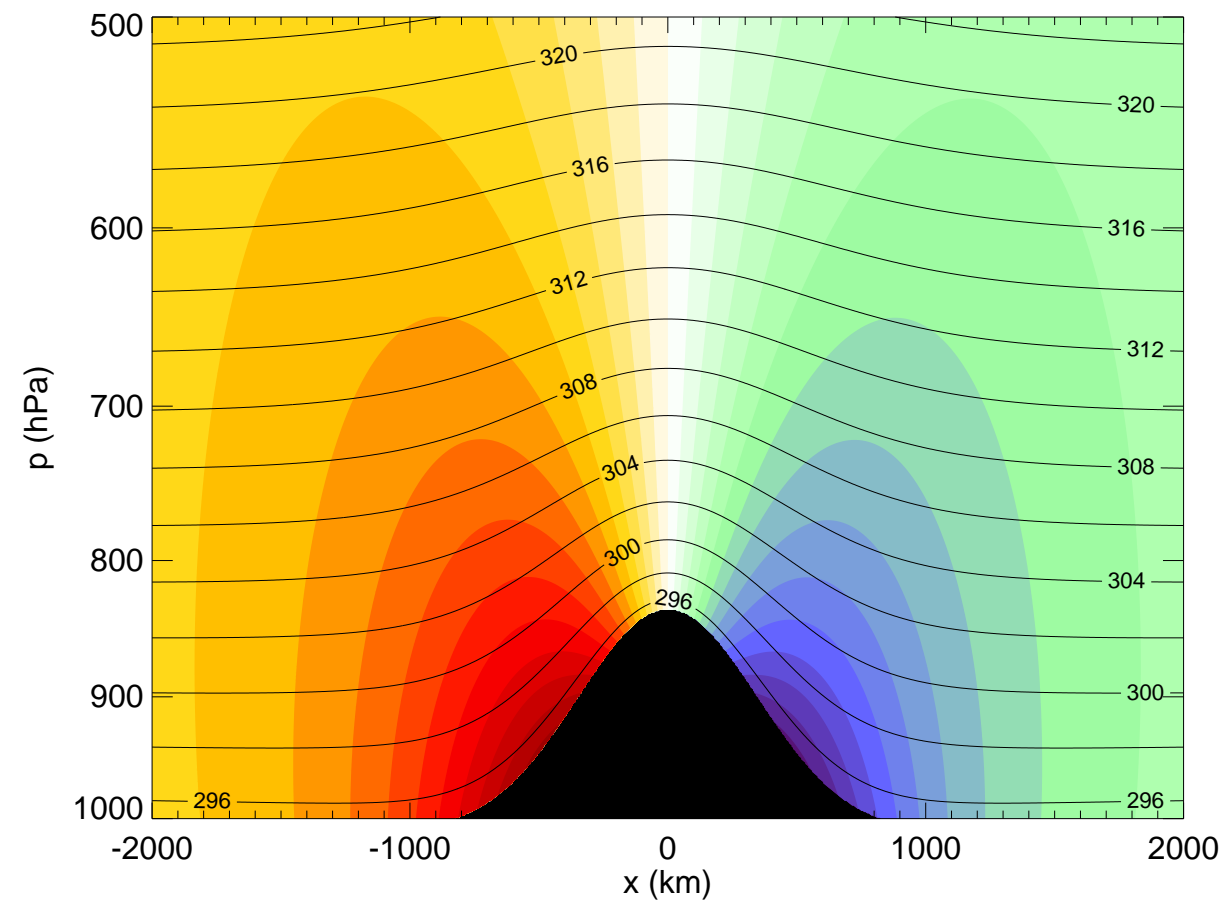

Figure 5.1: Meridional wind field (shading, warm colors are positive, cool colors negative, contour interval $=1 \mathrm{~m} / \mathrm{s}$ ) for an isentropic lower boundary computed using over-relaxation. The maximum height of the Gaussian mountain is $1700 \mathrm{~m}$ and its halfwidth $=500 \mathrm{~km}$. The maximum winds are $14.3 \mathrm{~m} / \mathrm{s}$.

We solve the discrete equations (5.7), (5.9), and (5.10) using the following standard successive over-relaxation (SOR) procedure. The current estimate of $M_{j, k}$ is denoted by $\hat{M}_{j, k}$, but should 
not be confused with the use of the 'hat' notation in chapter 3 to denote the spectral space component of a variable. From the current solution estimate $\hat{M}_{j, k}$, sweeping through the grid in lexicographic order, we first compute the current estimate of density from

$$
\hat{\rho}_{j, k}=\frac{p_{0}}{R \theta_{k}}\left(\frac{\hat{M}_{j, k+1}-\hat{M}_{j, k-1}}{c_{p} 2 \Delta \theta}\right)^{(1-\kappa) / \kappa},
$$

the current estimate of the dimensionless coefficient from

$$
\hat{A}_{j, k}= \begin{cases}\frac{g(\Delta \theta)^{2}}{f \theta_{k} \hat{\rho}_{j, k} P_{j, k}(\Delta x)^{2}} & \text { if } \theta_{S}\left(x_{j}\right)<\theta_{k}<\theta_{T} \\ 0 & \text { if } \theta_{B}<\theta_{k}<\theta_{S}\left(x_{j}\right)\end{cases}
$$

and then the current residual from

$$
\begin{aligned}
\hat{r}_{j, k} & =\hat{M}_{j, k-1}+\hat{M}_{j, k+1} \\
& +\hat{A}_{j, k}\left[(f \Delta x)^{2}+\hat{M}_{j-1, k}+\hat{M}_{j+1, k}\right] \\
& -2\left(1+\hat{A}_{j, k}\right) \hat{M}_{j, k} .
\end{aligned}
$$

The solution estimate on the interior is then updated by

$$
\hat{M}_{j, k} \leftarrow \hat{M}_{j, k}+\frac{\omega \hat{r}_{j, k}}{2\left(1+\hat{A}_{j, k}\right)}
$$

where $\omega$ is the overrelaxation factor and (5.13) and (5.14) are computed at the grid points $1 \leq j \leq$ $J-1,1 \leq k \leq K-1$. Finally, the top and bottom boundary points are updated from the boundary conditions (5.9) and (5.10), written in the form

$$
\begin{gathered}
\hat{M}_{j, K} \leftarrow \hat{M}_{j, K-1}+\Pi_{T} \Delta \theta \quad \text { for } \quad 1 \leq j \leq J-1, \\
\hat{M}_{j, 0} \leftarrow \frac{\left(\theta_{B} / \Delta \theta\right) \hat{M}_{j, 1}+\phi_{S}\left(x_{j}\right)}{1+\left(\theta_{B} / \Delta \theta\right)} \text { for } \quad 1 \leq j \leq J-1 .
\end{gathered}
$$

Equations (5.11)-(5.16) are iterated, starting with the initial estimate $\hat{M}_{j, k}=\tilde{M}\left(\theta_{k}\right)$. This initial estimate does not change on the lateral boundaries $j=0$ and $j=J$. The number of iterations can be increased until the desired level of accuracy is attained.

The above iterative procedure determines the Montgomery potential in the entire domain for a given geopotential $\phi_{S}(x)$ and a given potential temperature $\theta_{S}(x)$ along the lower surface. From 
the Montgomery potential the wind field can be easily recovered using geostrophic balance, and the pressure field can be computed using the hydrostatic approximation. The wind field can now be computed throughout the entire domain for a specific forcing function.

Figure 5.1 shows the solution computed for an isentropic ridge of the same height as the ridge shown in Fig. 3.3. A comparison of these two figures shows that the solution derived using Green's functions (3.41-3.42) and the solution found with the above iterative procedure give similar results. These figures are not expected to be identical because Fig. 3.3 was computed assuming the density was equal to the far-field density (only depends on $\theta$ ) while Fig. 5.1 was computed with density as a function of $\theta$ and $x$.

\section{$5.2 \quad$ Results}

All of the wind fields shown here were computed in $(x, \theta)$-space and then interpolated to $(x, p)$-space. In many cases a particular wind field is shown in both $(x, \theta)$-space and $(x, p)$-space. This provides two different views of the same information and hopefully makes interpretation easier. Thinking of the atmospheric response to forcing in a domain with $\theta$ as the vertical coordinate and massless layers along the lower boundary is somewhat abstract. In $(x, \theta)$-space the massless layer appears as a mountain along the lower boundary. This is not a physical mountain, but simply shows the regions where isentropic $(\theta)$ surfaces pass under the ground in physical space. When viewing a figure plotted in $(x, p)$-space, if the lowest pressure surface is uniform the massless layer does not appear and the isentropes intersect the lowest pressure surface. Often, the lowest pressure surface is not uniform and the massless layer appears as a mountain that represents locations where pressure surfaces are not defined. The shapes representing the massless layer in $(x, \theta)$-space and $(x, p)$-space are different from each other, and neither corresponds exactly to the actual orography in physical

space. Comparing figures in pressure coordinates with those in $\theta$ coordinates is a useful way to develop an intuition of the fluid response to obstacles.

All of the figures in this chapter (and Fig. 3.3) show the wind field as shaded contours in 1 $\mathrm{m} / \mathrm{s}$ intervals with warm colors representing positive $v$ (into the page) and cool colors representing 

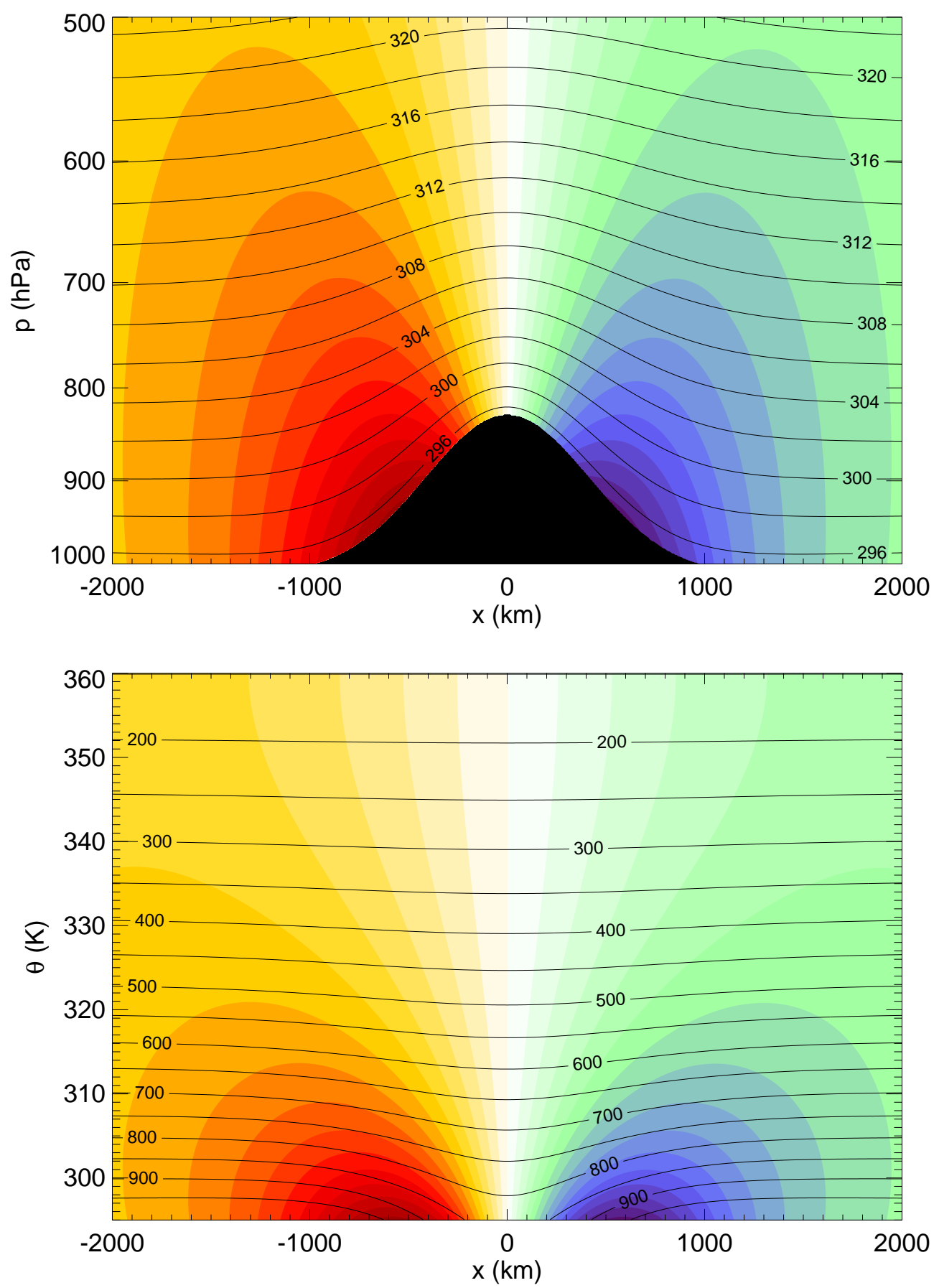

Figure 5.2: Meridional wind field (shading, $1 \mathrm{~m} / \mathrm{s}$ intervals) for an isentropic ridge with $H=1800$ $\mathrm{m}, v_{\max }=15.4 \mathrm{~m} / \mathrm{s}$. Black contours are isentropes (top) and isobars (bottom). 

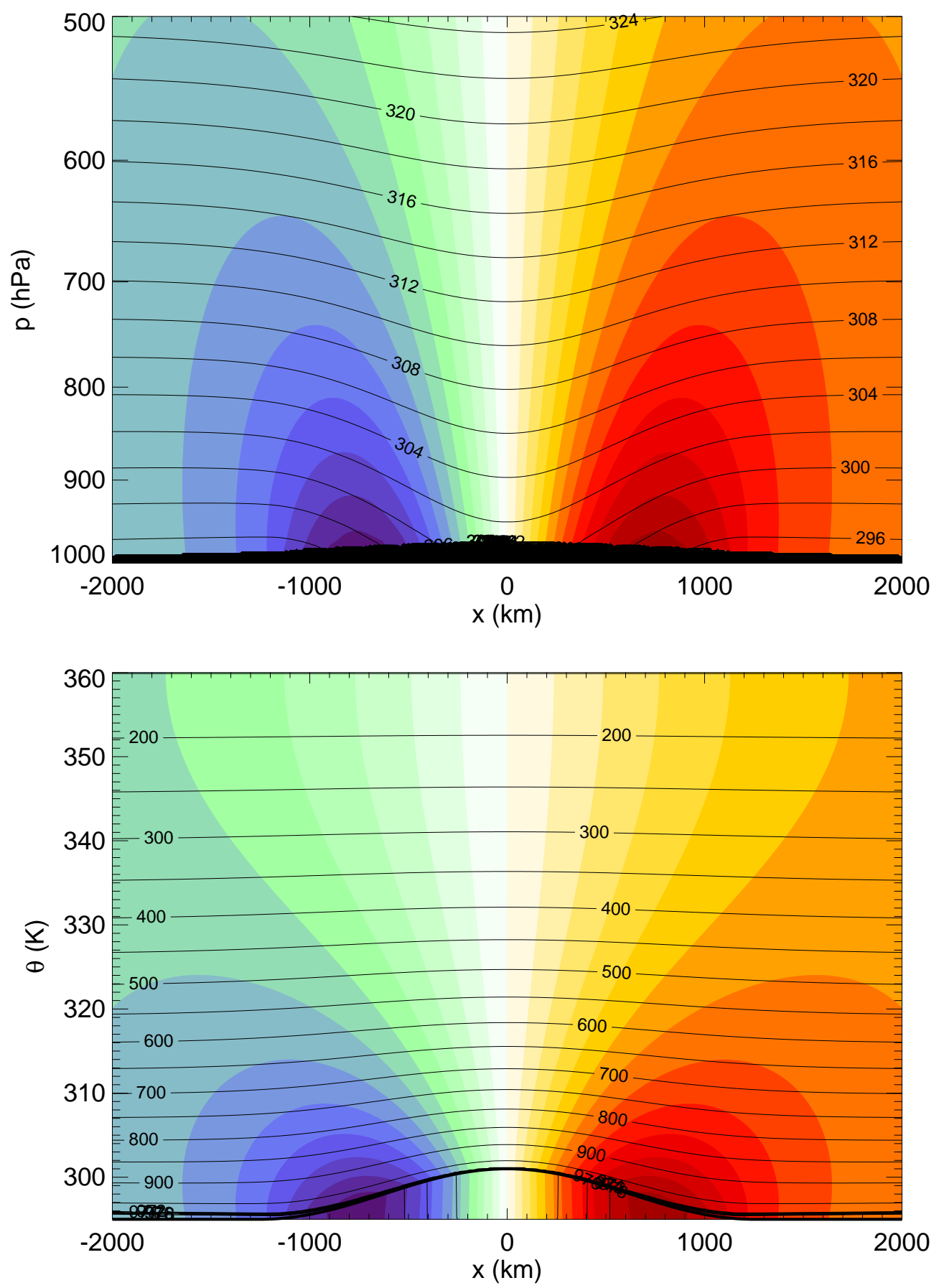

Figure 5.3: Meridional wind field (shading, $1 \mathrm{~m} / \mathrm{s}$ intervals) for a heated flat lower surface with a 6 $\mathrm{K}$ temperature anomaly and Gaussian halfwidth $\mathrm{a}=600 \mathrm{~km}, v_{\max }=12.7 \mathrm{~m} / \mathrm{s}$. The massless layer in $(x, p)$-space (top) is indicated by black object along lower boundary, in $(x, \theta)$-space (bottom) it is indicated by the thick black line. Contour spacing in the massless layer is $1 \mathrm{hPa}$. 

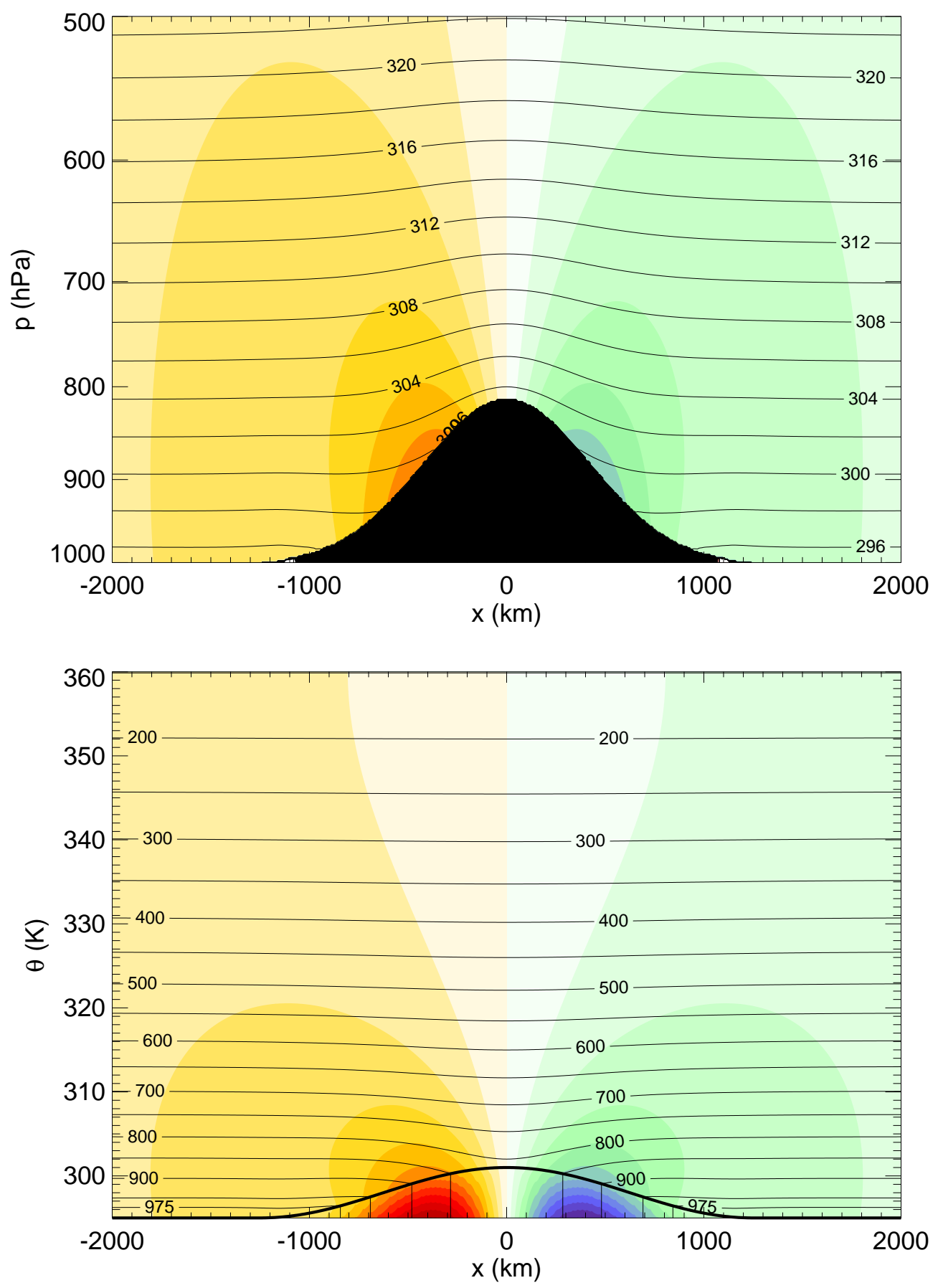

Figure 5.4: Meridional wind field (shading, $1 \mathrm{~m} / \mathrm{s}$ intervals) for a heated ridge with a $6 \mathrm{~K}$ temperature anomaly, $H=1800 \mathrm{~m}, a=600 \mathrm{~km}, v_{\max }=11.13 \mathrm{~m} / \mathrm{s}$. The massless layer is indicated by the black object (top) and the thick black line (bottom). Halfwidth of ridge is $900 \mathrm{~km}$. Contour spacing in the massless layer is $50 \mathrm{hPa}$ except for lowest contour of $975 \mathrm{hPa}$. 

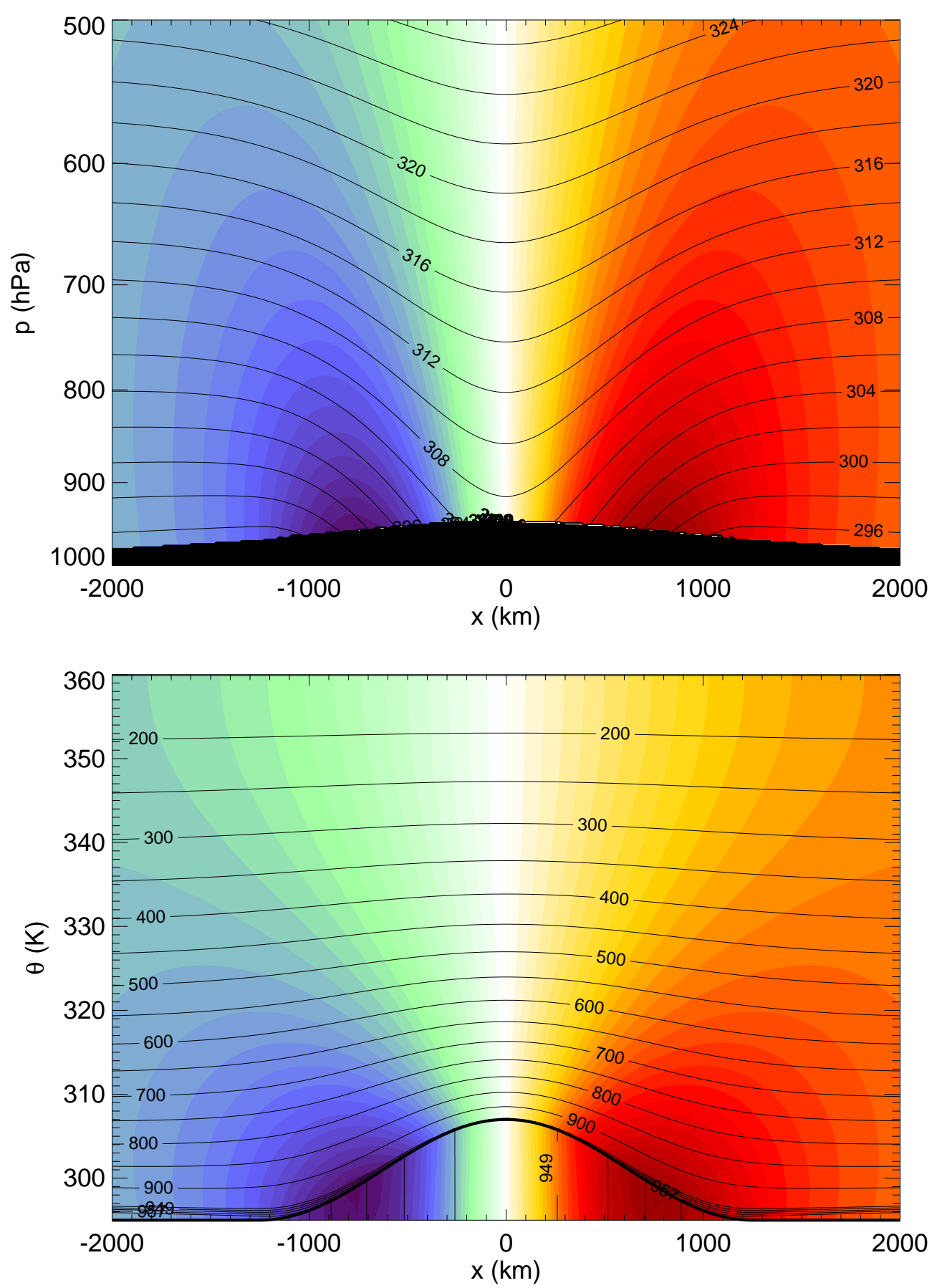

Figure 5.5: Same as Fig. 5.3 except with a $12 \mathrm{~K}$ temperature anomaly, $v_{\max }=27.27 \mathrm{~m} / \mathrm{s}$. Contour spacing in the massless layer is $4 \mathrm{hPa}$. 

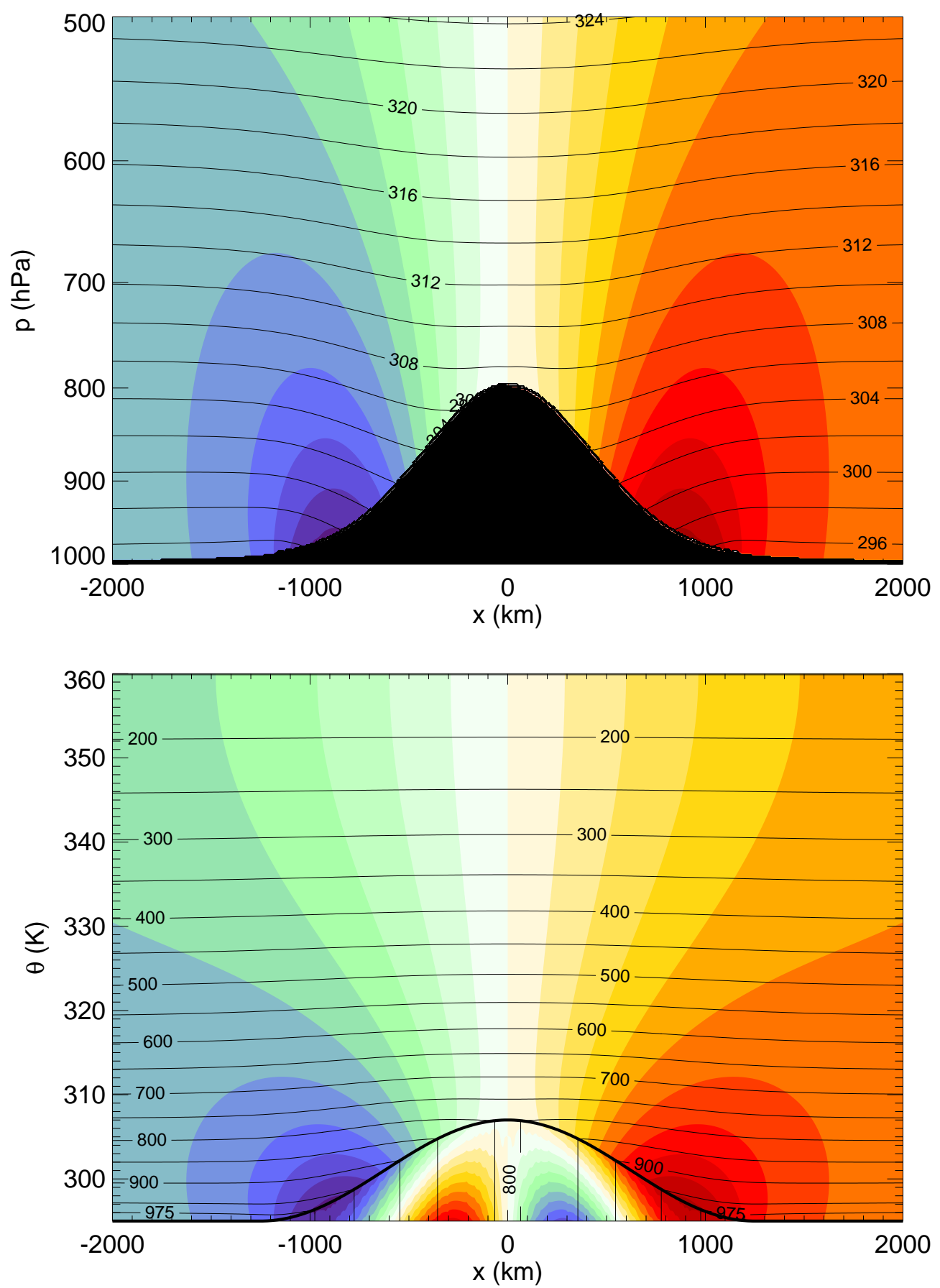

Figure 5.6: Same as Fig. 5.4 except with a $12 \mathrm{~K}$ temperature anomaly, $v_{\max }=10.3 \mathrm{~m} / \mathrm{s}$. 
negative $v$ (out of the page). For the figures shown in $(x, p)$-space the black contours show isentropes (surfaces of constant potential temperature) in intervals of $2 \mathrm{~K}$. The figures shown in $(x, \theta)$-space have isobars contoured in black.

Figures 5.2-5.6 show the wind fields that result from the three simple cases discussed previously and represented in Fig 1.1. An isentropic ridge with a crest height of $1800 \mathrm{~m}$ is shown in Fig. 5.2 and can be clearly identified by the fact that the isentropes do not intersect the ridge and the flow is anticyclonic. A flat lower boundary with a potential temperature anomaly of $6 \mathrm{~K}$ is shown in Fig. 5.3, which results in a $12.7 \mathrm{~m} / \mathrm{s}$ cyclonic flow. Figure 5.4 combines these two forcing cases into a heated ridge with a crest height of $1800 \mathrm{~m}$ and a heating anomaly of $6 \mathrm{~K}$.

Note that the isentropic lower surface has anticyclonic flow anchored over the ridge, but for the case of the flat lower boundary the flow is cyclonic. The heated ridge with a temperature anomaly of $6 \mathrm{~K}$ has anticyclonic flow but when the temperature anomaly is $12 \mathrm{~K}$ the flow becomes cyclonic. This demonstrates the competing influences of the purely orographic forcing and the temperature forcing. Once the surface temperature anomaly has become strong enough to overcome the orographically forced tendency towards anticyclonic motion, the wind field produced by a heated ridge grows with the tempterature anomaly. The results also demonstrate (not shown) that, as the width of the ridge grows for a set temperature anomaly, the influence of the anomaly is spread out and effectively acts as a weaker anomaly.

It is also apparent in $(x-\theta)$-space that the isentropic ridge case does not contain a massless layer, but for the heated flat lower boundary and heated ridge a massless layer is present and marked as the area below the thick black line. Pressure in the massless layer is independent of $\theta$, as is clear from the vertical isobars within the massless layers of Figs. 5.3 and 5.4. This is required by the hydrostatic approximation. Although the pressure is uniform in $\theta$ within the massless layer, it varies strongly as a function of $x$, which requires a nonzero wind field. The velocity is required to satisfy the lower boundary condition given by (5.6), which can be seen as the slope of the velocity increases with the magnitude of the velocity along the lower boundary. The concept of nonzero velocity underneath the surface of the ground is admittedly strange but is valid based on the governing 
equations. Because there is zero mass within this region of the domain, there can be no mass flux regardless of the structure of the velocity field inside the massless layer.

For the isentropic case potential vorticity is conserved. Combining this with the use of isentropic coordinates leads to an intuitive explanation for the wind field response to the mountain as seen in Fig. 5.2. Recall that, in the absence of heating, mass cannot cross the isentropic surfaces. This constrains how the mass field must adjust when the isentropes are moved up or down. One of the primary benefits to using isentropic coordinates is the simplicity of the expression for potential vorticity. The denominator of the potential vorticity is given by the pseudodensity $-(1 / g)(\partial p / \partial \theta)$, which becomes smaller when the isentropes are compressed as over the crest of the isentropic ridge. Because the potential vorticity is conserved, the decrease in magnitude of the denominator implies the numerator also must decrease. The only part of the numerator that can decrease in magnitude is $\partial v / \partial x$, so a negative meridional velocity gradient is required, which is exactly what Fig. 5.2 shows. It is apparent that the velocity gradient is greatest where the isentropes are the most compressed. Similar reasoning can be applied to the case of stretched isentropes with the only difference being the sign of the velocity gradient. The isentropic structure for the cases with a heated lower boundary is quite different. The heating forces mass across isentropic surfaces. As radiation heats the surface, an upslope motion is generated. When the mass moves upward it is transferred from one isentropic layer to next warmer layer. This is what physically causes the isentropes to bend down towards the heated surface. The stretched isentropic layers generate a wind field that is oriented opposite to that which results from the isentropic case with compressed isentropes.

Each of figures 5.2-5.3 show the wind maximum to be in the lowest layers of the fluid and to decay rapidly in the vertical and horizontal directions. This matches well with a basic description of a low-level jet. In agreement with the insights offered by the invertibility principle, all three figures show an increased gradient in the velocity field when the isentropes are either compressed or stretched out. These figures clearly indicate that in the absence of other factors a (sufficiently) diabatically heated surface will result in a cyclonic wind field. It is also clear that the jets of opposite sign on either side of the ridge are two components of the same response to the forcing. A heated 
ridge does not lead to a single LLJ, but two LLJs that form a cyclonic or anticyclonic circulation.

\subsection{Technical Discussion}

Solving this system by relaxing finite difference approximations of the continuous equations highlights two aspects of the problem. The first is that the grid representing our domain is not in general isotropic. This most likely slows down the relaxation procedure and necessitates going through many more iterations than would be required for an isotropic grid. If the grid spacing in the vertical and horizontal is carefully chosen to result in the coefficient $A$ being approximately equal to 1 , the grid outside the massless layer is nearly isotropic. Within the massless layer $A$ equals zero and the grid will not be isotropic regardless of the grid spacing. The second complication arises because the system is strongly coupled in the vertical dimension of the massless layer, but only indirectly coupled in the horizontal direction. For Gauss-Seidel over relaxation using lexicographic ordering this also leads to very slow convergence. It is possible that using line relaxation in the vertical would help this. Both of these issues cause the iteration procedure to be slow, with many iterations necessary before the residual can be said to be small.

The computed value of the residual needs to be put in a meaningful context to determine how many iterations of the solution procedure are necessary. Often the magnitude of the residual can be compared to the magnitude of the forcing. Applying this method here is not straightforward because the forcing is only present in part of the domain, but the residual is defined at each grid point. We have chosen to compare the norm of the residual after a single iteration to the norm of the residual for a later iteration. When this norm has decreased by about 2 orders of magnitude the wind field no longer significantly changes with further iterations. Most of the figures shown have been iterated until the residual has decreased by more than two orders of magnitude. Overrelaxation parameters of around 1.7 to 1.95 have been used. It is difficult to theoretically estimate an optimal overrelaxation parameter because our domain is composed of an isotropic region and a non-isotropic region. The values used were obtained through a series of numerical tests. 


\section{Chapter 6}

\section{CONCLUSIONS}

\subsection{Overview}

The research presented in this dissertation shows that heated topographic features are an important component to the forcing of atmospheric low-level jets. This hypothesis is demonstrated by calculating the balanced response of a stratified fluid to forcing along the lower boundary. The forcing used here is intended to mimic atmospheric obstacles and surface heating. The results clearly show that the wind field generated by a heated ridge includes LLJs of opposite sign on either side of the ridge.

The basic method used is very simple. The forcing takes the form of a potential vorticity anomaly that is determined by the boundary conditions along the lower boundary. This potential vorticity is then used to compute the wind field through the invertibility principle that is derived in chapters 3, 4, and 5. Figure 1.1 shows the three cases this method was applied to: an isentropic ridge along the lower boundary, a heated lower boundary with a flat geopotential surface, and a heated ridge. To the best of the author's knowledge, the potential vorticity invertibility principle in isentropic coordinates has not previously been solved when the lower boundary includes mountains.

Three separate methods have been used to solve the invertibility principle. Chapter 3 finds solutions by using Fourier transforms in the horizontal dimension to derive a vertical structure equation that is then solved using the appropriate Green's functions. Analytical solutions are plotted for the wind field that is forced by an isentropic ridge. Assuming the fluid to satisfy hydrostatic balance and requiring $\theta$ to increase monotonically with height an expression for the critical crest height is 
derived. Ridges that are higher than the critical crest height must penetrate the lower isentropes in the domain while ridges with a height below this value can remain isentropic if no heating is present. This provides a generalization of the theory presented by Eliassen (1980). Chapter 4 uses a similar approach to solve the invertibility principle but vertical transforms are used to derive a horizontal structure equation. This derivation is generalized to a sphere and the horizontal structure equation is solved using spheroidal harmonics. The analytical solutions derived in chapters 3 and 4 are only closed form solutions for the case of an isentropic lower surface. When heating is introduced along the lower boundary of an isentropic domain a massless layer is inevitable and the solutions must be found iteratively. The third method for solving the invertibility principle was to use finite difference approximations of the governing equations which are then solved in chapter 5 using Gauss-Seidel over-relaxation.

Another useful result of this research is the practical presentation of the massless layer. It is intended that this work will help to clarify how the massless layer can be incorporated into the solution of other problems in a straightforward manner.

\subsection{Summary of wind field response to forcing}

Isentropic ridges produce an anticyclonic wind field. The closer the ridge height comes to the critical crest height the tighter the isentropes are packed over the ridge and the stronger the corresponding wind field is. In contrast, the case of a heated flat lower boundary results in a cyclonic wind field. When these two cases are combined into the case of a heated ridge the total wind field is decreased due to the competing impacts of the isentropic orography and the heating. For temperature anomalies that are quite small the mechanical influence of the ridge is dominant and the wind field is anticyclonic. However, for temperature anomalies that are closer to those observed (in YOTC) the heating along the lower boundary is the dominant factor and the wind field is cyclonic. The heated ridge shown in Fig. 5.4, with a temperature anomaly of $6 \mathrm{~K}$, has an anticyclonic flow, while the heated ridge shown in Fig. 5.6 with a temperature anomaly of $12 \mathrm{~K}$, has a cyclonic flow. Byerle and Paegle (2003) noted a change from cyclonic to anticyclonic flow during the North American 
winter. They attributed this to a change in the zonal flow and the interaction with the orography. The results here suggest this transition is due to the winter temperature anomaly dropping below the critical value that is needed for a cyclonic circulation.

The solutions computed in chapter 5 also show that in general the wind field will be stronger for a stronger temperature anomaly when the width and height of a ridge are constant. Similarly, for a constant magnitude of the temperature anomaly, the wind field will decrease in strength as the width of the ridge is increased.

The persistent occurrence of LLJs in the atmosphere has led to many ideas concerning the possible mechanisms for their development. Blackadar (1957) hypothesized that the jets are due to an inertial oscillation acting on the diurnal cycle of boundary layer eddy viscosity while Holton (1967) proposed that a buoyancy-driven flow over sloping terrain was the primary process responsible for the jets. Wexler (1961) claimed the GPLLJ is an analogue to the western boundary currents of the ocean basins. The North and South American LLJs are also often attributed to a simple deflection of the trade winds by the orography. Each of these mechanisms are important to the LLJs observed in North and South America but no individual mechanism can entirely explain their generation, maintenance, and variability. The work presented here shows that heated orography also needs to be included in this list of influential mechanisms for LLJs.

The basic characteristics of the wind field from the YOTC data shown in Figs. 2.4-2.7 agrees well with the results shown in Fig. 5.6. The orientation of the wind field (cyclonic) is the same and the magnitude is similar to that shown by the YOTC data. The figures in chapter 5 can be interpreted as representing means of roughly 1-3 months. If the temperature distribution along the lower boundary is made larger, say $20 \mathrm{~K}$ (see Jiang et al. 2010), the wind speeds are closer to what would be expected for daily or weekly mean fields. The parameters used to compute all of the wind fields shown in chapter 5 were chosen to be comparable to Earth-like mountains with realistic temperature anomalies along the lower boundary. It is probably not appropriate to make direct comparisons between the results of chapter 5 and observations of specific jet events or even climatologies because the assumptions of a steady, nonviscous fluid will certainly lead to discrepancies between the 
computed and observed wind fields, especially along the lower boundary, which is precisely where the LLJ maxima in chapter 5 are located.

The YOTC data of the meridional wind field and potential temperature distribution for two Rocky Mountain latitudes and two Andes latitudes are intended to give a general idea of the mean wind and temperature distributions during the warm season of those regions. The results computed in chapter 5 show general agreement with the basic wind and temperature characteristics. However, a detailed comparison between the results of this research and observations of atmospheric LLJs is beyond the scope of this work.

\subsection{Final Discussion}

Contributions to our understanding of fluid systems made by this research can be divided into two components. First, the general question of how a stratified fluid responds to a non-trivial lower boundary has been solved. The isentropic coordinate invertibility principle was solved for the case of a domain including a massless layer. This can be applied to a variety of interesting problems in future research. The second component is the application of the previously mentioned solutions to the study and interpretation of atmospheric LLJs.

There are several mechanisms that are known to be important to the generation of LLJs. These include the kinematic redirecting (deflection) of the flow by mountains, the inertial oscillation acting with the fluctuating boundary layer turbulence, and the pressure gradient force. Often these mechanisms act on a regional scale and only influence one side of a mountain range at a given time. As a result, past studies have generally studied each LLJ in isolation. This research makes it clear that a heated mountain influences jets on both flanks which indicates a strong connection between the LLJs on opposite sides of the Rocky Mountains and the Andes.

Because a steady state has been assumed the time evolution of potential vorticity and wind can obviously not be seen. This model only computes the wind field that results from the potential vorticity at an instant in time. Alternately, this could be interpreted as the potential vorticity averaged over a time interval. This does not imply the wind field for the chosen time or period is less accurate; 
it simply means that we cannot say anything about the potential vorticity field before or after the chosen time interval.

It may seem that in the analytical parts of this research the system was overly simplified by assuming symmetry in $x$, that density only varies as a function of $\theta$, and idealized orographic shapes. When the solutions are found through finite difference approximations these simplifications are unnecessary and can be dropped. Particular scenarios that are more complex than the analytic derivation allowed for can then be studied. Having first derived analytical solutions for simple cases, the solutions found through iteration can be more readily interpreted.

This research lays the groundwork for future projects in several directions. The efficiency of the finite difference solution method could most likely be increased. The system of weaklynonlinear equations that result from the finite-difference solution of the invertibility principle is quite sparse. This suggests that a solution method such as the conjugate gradient method may work well. Developing an algorithm that utilizes multi-grid methods is also possible and would almost certainly converge to the correct solution faster than does the over-relaxation method used here.

One potential future project would be to carry out a similar study with more complex topography, possibly in spherical coordinates. If the topography being studied is more complex, the heating along the lower boundary should also be adjusted and will probably need to be more complex as well. The results here have been presented in a fairly general manner but they could be used for particular case studies. The atmospheric response to heated orography is clearly important to monsoon circulations. It would be interesting to study particular surface heating profiles and orographic structures in this context. Specific case studies could also be made of the Rocky Mountains or Andes using data from particular months and latitudes similar to the YOTC data shown in chapter 2. This would offer insight into the importance of the surface heating to the LLJs relative to other forcing mechanisms active at that time. 


\section{BIBLIOGRAPHY}

Abramowitz, M. and I. A. Stegun, 1965: Handbook of Mathematical Functions. Dover. 1046 pp.

Arakawa, H., 1957: Characteristics of the low-level jet stream. J. Meteor., 13(5), 504-506.

Blackadar, A. K., 1957: Boundary layer wind maxima and their significance for the growth of nocturnal inversions. Bull. Amer. Meteor. Soc., 38, 283-290.

Bonner, W. D., 1968: Climatology of the low level jet. Mon. Wea. Rev., 96, 833-850.

Buajitti, K. and A. K. Blackadar, 1957: Theoretical studies of diurnal wind-structure variations in the planetary boundary layer. Quart. J. Roy. Meteor. Soc., 83, 486-500.

Byerle, L. A. and J. Paegle, 2002: Description of the seasonal cycle of low-level flows flanking the Andes and their interannual variability. Meteorologica, 27, 71-88.

Byerle, L. A. and J. Paegle, 2003: Modulation of the Great Plains low-level jet and moisture transports by orography and large-scale circulations. J. Geophys. Res., 108. doi: 10.1029/2002JD003005.

Chao, S.-Y., 1985: Coastal jets in the lower atmosphere. J. Phys. Oceanogr., 15, 361-371.

Eliassen, A., 1980: Balanced motion of a stratified, rotating fluid induced by bottom topography. Tellus, 32, 537-547.

Farquharson, S. J., 1939: The diurnal variation of wind over tropical Africa. Quart. J. Roy. Meteor. Soc., 65, 165-183.

Flammer, C., 1957: Spheroidal Wave Functions. Stanford University Press. 84 pp.

Fulton, S. R. and W. H. Schubert, 1991: Surface frontogenesis in isentropic coordinates. J. Atmos. Sci., 48(23), 2534-2541.

Higgins, R. W., Y. Yao, E. S. Yarosh, J. E. Janowiak, and K. C. Mo, 1997: Influence of the Great Plains low-level jet on summertime precipitation and moisture transport over the central United States. J. Climate, 10, 481-507.

Hoecker, Jr., W. H., 1963: Three southerly low-level jet systems delineated by the Weather Bureau special pibal network of 1961. Mon. Wea. Rev., 63(10), 573-582.

Holton, J. R., 1967: The diurnal boundary layer wind oscillation above sloping terrain. Tellus, 19, 199-205. 
Houze, Jr., R. A., 2004: Mesoscale convective systems. Rev. Geophys., 42. RG4003, doi: 10.1029/2004RG000150.

Hoxit, L. R., 1975: Diurnal variations in planetary boundary-layer winds over land. Bound.-Lay. Meteorol., 8(1), 21-38.

Hsu, Y.-J. G. and A. Arakawa, 1990: Numerical modeling of the atmosphere with an isentropic vertical coordinate. Mon. Wea. Rev., 118, 1933-1959.

Jiang, Q., S. Wang, and L. O'Neill, 2010: Some insights into the characteristics and dynamics of the Chilean low-level coastal jet. Mon. Wea. Rev., 138, 3185-3206. doi:10.1175/2010MWR3368.1.

Jiang, X., N.-C. Lau, I. M. Held, and J. J. Ploshay, 2007: Mechanisms of the Great Plains low-level jet as simulated in an a GCM. J. Atmos. Sci., 64(2), 532-547. doi:10.1175/JAS3847.1.

Kanamitsu, M., W. Ebisuzaki, J. Woollen, S.-K. Yang, J. J. Hnilo, M. Fiorino, and G. L. Potter, 2002: NCEP-DOE AMIP-II reanalysis (R-2). Bull. Amer. Meteor. Soc., 83, 1631-1643. doi:10.1175/BAMS-83-11-1631.

Laing, A. G. and J. M. Fritsch, 1997: The global population of mesoscale convective complexes. Quart. J. Roy. Meteor. Soc., 123, 389-405.

Lorenz, E. N., 1955: Available potential energy and the maintenance of the general circulation. Tellus, 8, 157-167.

Macklin, S. A., N. A. Bond, and J. P. Walker, 1990: Structure of a low-level jet over lower Cook Inlet, Alaska. Mon. Wea. Rev., 61, 2568-2578.

Maddox, R. A., 1980: Mesoscale convective complexes. Bull. Amer. Meteor. Soc., 61, 1374-1387.

Marengo, J. A., W. R. Soares, C. Saulo, and M. Nicolini, 2004: Climatology of the low-level jet east of the Andes as derived from the NCEP-NCAR reanalysis: Characteristics and temporal variability. J. Climate, 17, 2261-2280.

Means, L. L., 1952: On thunderstorm forecasting in the central United States. Mon. Wea. Rev., 80(10), 165-189.

Mitchell, M. K., R. W. Arritt, and K. Labas, 1995: An hourly climatology of the summertime Great Plains low-level jet using wind profiler observations. Wea. Forecasting, 10, 576-591.

Muñoz, R. C. and R. D. Garreaud, 2005: Dynamics of the low-level jet off the west coast of subtropical South America. Mon. Wea. Rev., 133, 3661-3677.

Nogués-Paegle, J., J. Paegle, and Coauthors, 2001: American low-level jets - a scientific prospectus and implementation plan. Available online at http://www.clivar.org/organization/vamos/Publications/alls.pdf.

Reiter, E. R., 1963: Jet-Stream Meteorology. The University of Chicago Press. 515 pp.

Rife, D. L., J. O. Pinto, A. J. Monaghan, C. A. Davis, and J. R. Hannan, 2010: Global distribution and characteristics of diurnally varying low-level jets. J. Climate, 23, 5041-5064. doi: 10.1175/2010JCLI3514.1. 
Saulo, A. C., M. Nicolini, and S. C. Chou, 2000: Model characterization of the South American low-level flow during the 1997-1998 spring-summer season. Clim. Dynam., 16, 867-881.

Schubert, W. H. and M. T. Masarik, 2006: Potential vorticity aspects of the MJO. Dynam. Atmos. Oceans, 42, 127-151. doi: 10.1016/j.dynatmoce.2006.02.003.

Schubert, W. H., R. K. Taft, and L. G. Silvers, 2009: Shallow water quasi-geostrophic theory on the sphere. J. Adv. Model. Earth Syst., 1(2), 17 pp. doi: 10.3894/JAMES.2009.1.2.

Starr, V. P., 1945: A quasi-lagrangian system of hydrodynamical equations. J. Meteor., 2, 227-237.

Stensrud, D. J., 1996: Importance of low-level jets to climate: A review. J. Climate, 9, 1698-1711.

Stensrud, D. J., M. H. Jain, K. W. Howard, and R. A. Maddox, 1990: Operational systems for observing the lower atmosphere: Importance of data sampling and archival procedures. $J$. Atmos. Ocean. Technol., 7, 930-937.

Tarasova, T. A., J. P. R. Fernandez, I. A. Pisnichenko, J. A. Marengo, J. C. Ceballos, and M. J. Bottino, 2006: Impact of new solar radiation parameterization in the eta model on the simulation of summer climate over South America. J. Appl. Meteor. Climatol., 45, 318-333.

Vera, C., J. Baez, M. Douglas, C. Emmanuel, J. Marengo, J. Meitin, M. Nicolini, J. Nogues-Paegle, J. Paegle, O. Penalba, C. Saulo, M. Silva Dias, P. Silva Dias, and E. Zipser, 2006: The South American low-level jet experiment. Bull. Amer. Meteor. Soc., 87, 63-77.

Verkley, W. T. M., 2009: A balanced approximation of the one-layer shallow-water equations on a sphere. J. Atmos. Sci., 66, 1735-1748. doi: 10.1175/2008JAS2837.1.

Waliser, D. E., M. Moncrieff, D. Burridge, and Coauthors. The 'year' of tropical convection (May 2008 to April 2010): Climate variability and weather highlights. Bull. Amer. Meteor. Soc. submitted.

Wexler, H., 1961: A boundary layer interpretation of the low-level jet. Tellus, 13, 368-378.

Whiteman, C. D., X. Bian, and S. Zhong, 1997: Low-level jet climatology from enhanced rawinsonde observations at a site in the southern Great Plains. J. Appl. Meteor., 36, 1363-1376. 